\title{
Iron Uptake in Host Defense Against Pneumonia
}

\author{
Kathryn Michels \\ Gaithersburg, MD
}

B.A St. Mary's College of Maryland, 2012

M.S University of Virginia, 2014

A Dissertation Presented to the Graduate Faculty of the University of Virginia in Candidacy for the Degree of Doctor of Philosophy

Department of Microbiology, Immunology, and Cancer Biology University of Virginia May 2017 
Table of Contents

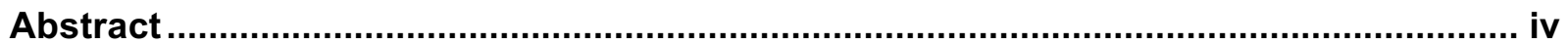

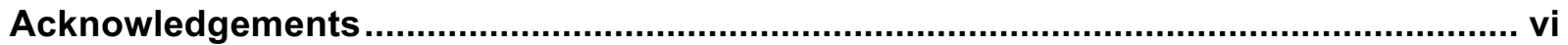

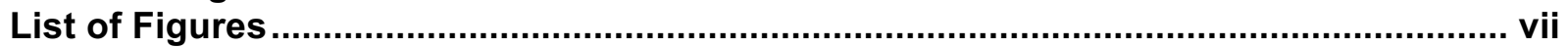

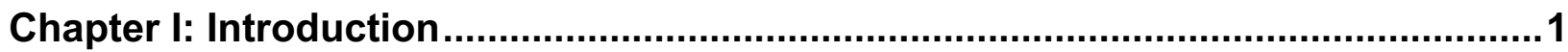

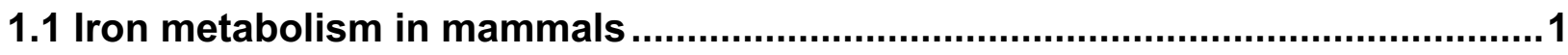

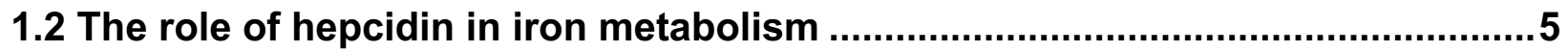

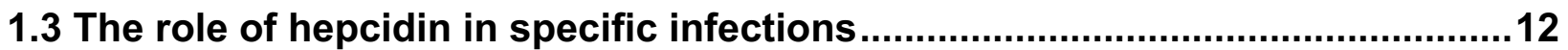

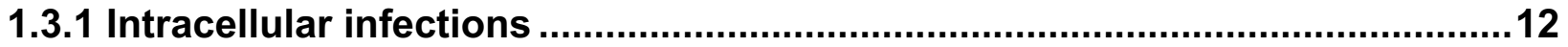

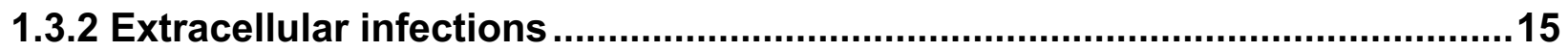

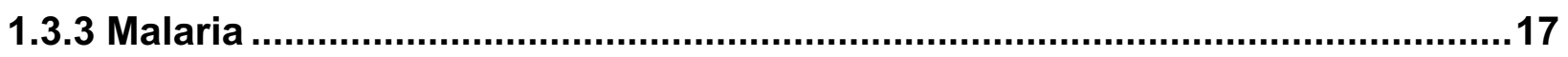

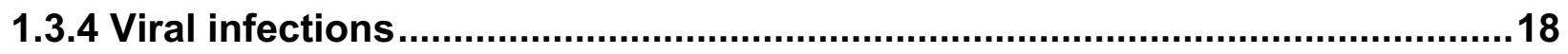

1.3.5 Iron-independent functions of hepcidin ......................................................19

1.5.4 Evidence that host iron levels are important in invasive aspergillosis

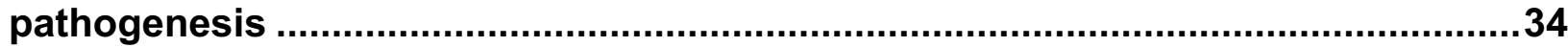

Chapter II: The role of hepcidin in Gram-negative pneumonia ...............................38

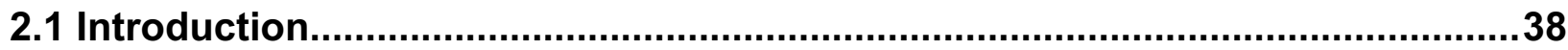

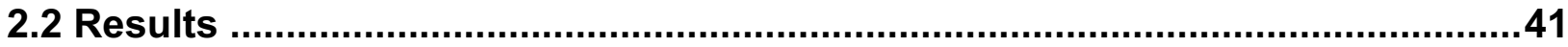

2.2.1 Plasma iron is suppressed during bacterial pneumonia ................................41

2.2.2 Increased iron availability worsens the outcome of infection ........................41

2.2.5 Excess iron promotes the growth of $K$. pneumoniae in plasma .....................60

2.2.6 Hepcidin-deficient mice have intact cellular immunity against Klebsiella ....64

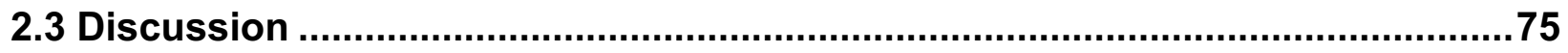

2.4 Methods

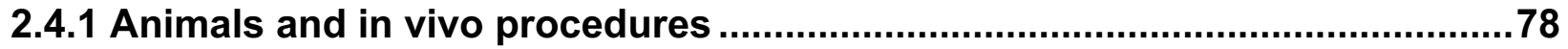

2.4.2 Tissue harvest and determination of bacterial burden...................................79

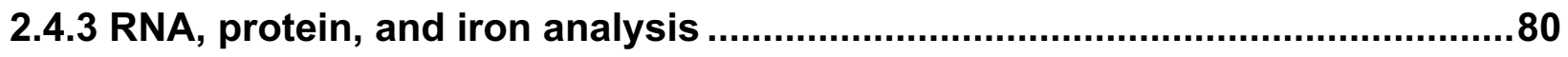

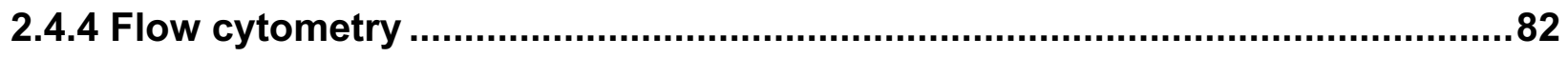




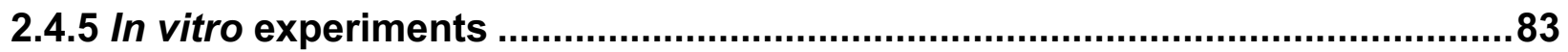

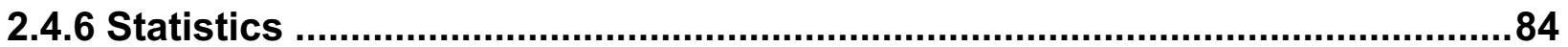

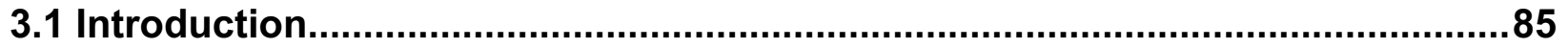

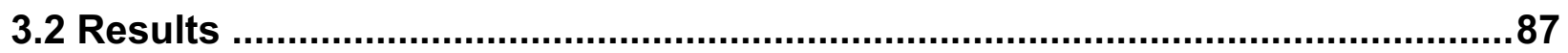

3.2.1 Infection with $A$. fumigatus induces pulmonary hemorrhage .......................87

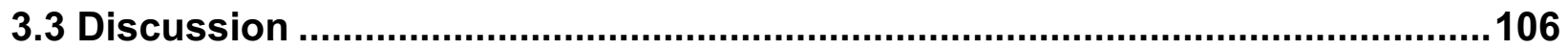

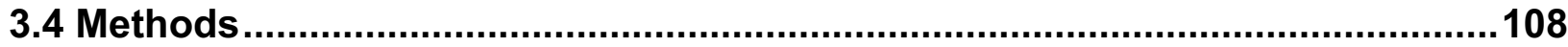

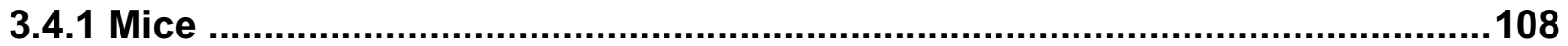

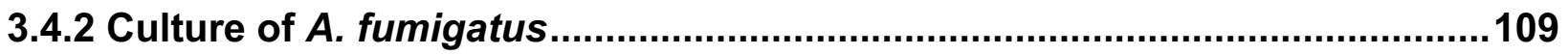

3.4.3 Protein, histology, and iron analysis...........................................................

Chapter IV: Concluding remarks and future directions ......................................112

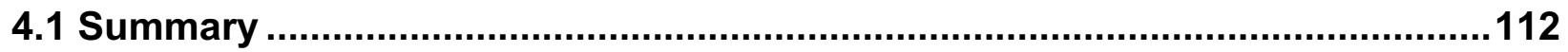

4.2 Is hepcidin dysregulation a component of cirrhosis-mediated susceptibility to

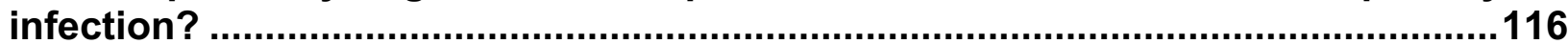

4.3 Do infections stimulate a release of iron from cells? ....................................126

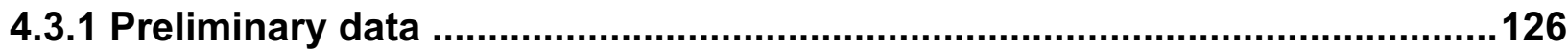




\section{Abstract}

Iron acquisition is a crucial component in microbial pathogenesis and determines the outcome of many diseases. This thesis examines the role of iron uptake in two distinct models of pneumonia caused by bacterial and fungal with the ultimate goal of identifying novel therapies. Gram-negative pneumonia is a dangerous illness which is difficult to treat due to antibiotic resistance among the causative pathogens. Hepcidin is the master regulator of extracellular iron availability in vertebrates, but its role in the context of host defense is undefined. We hypothesized that hepcidin-mediated depletion of extracellular iron during Gram-negative pneumonia protects the host by limiting dissemination of bacteria to the bloodstream. During experimental pneumonia, hepcidin was induced and mediated a rapid decline in plasma iron. In contrast, hepcidin-deficient mice developed a paradoxical increase in plasma iron during infection which was associated with profound susceptibility to bacteremia. Incubation of bacteria with ironsupplemented plasma enhanced bacterial growth in vitro, and systemic administration of iron to WT mice similarly promoted increased susceptibility to bloodstream infection. Finally, treatment with a hepcidin analogue restored hypoferremia in hepcidin-deficient hosts, mediated bacterial control, and improved outcomes. These data show hepcidin induction during pneumonia to be essential to preventing bacterial dissemination by limiting extracellular iron availability. Hepcidin agonists may represent an effective therapy for Gram-negative infections in patients with impaired hepcidin production or signaling.

Invasive pulmonary aspergillosis is a devastating fungal pneumonia which affects severely immunocompromised patients. Mortality remains high even with aggressive 
antifungal therapy, highlighting the need for more therapeutic options. It has been shown that high-affinity iron scavenging is crucial for the pathogenesis of invasive pulmonary aspergillosis, and that heme-containing proteins are necessary for Aspergillus hyphal growth and resistance to oxidative stress. The most abundant source of iron in the body is heme, but there are conflicting reports in the literature as to whether Aspergillus species are able to utilize heme. We hypothesized that heme uptake is important in the pathogenesis of invasive pulmonary aspergillosis. We found that, during the first 3 days of experimental invasive aspergillosis, both total and extracellular lung heme iron content increased as the infection progressed due to pulmonary hemorrhage and angioinvasion. We found that treatment with exogenous hemin, but not tin protoporphyrin, worsened the outcome of infection without appearing to promote fungal growth. In minimal media, exogenous hemin promoted $A$. fumigatus growth, conidiation, and resistance to oxidative stress in vitro. Tin protoporphyrin accumulated in the cell wall and cytosol of $A$. fumigatus hyphae. Together, these data suggest that $A$. fumigatus can utilize exogenous heme under certain circumstances and that heme uptake should be further explored as a virulence mechanism. 


\section{Acknowledgements}

Working in this lab has set my standards for what I consider to be a strong research group: a high set of standards for the quality of work and depth of critical thinking, and a deep-seated habit of helping your colleagues power through long and tedious experiments. Borna has guided me through experiment design, the stages of writing papers and proposals, and deciding how I want to continue my career. He is both rigorous in his expectations, and very warm and kind. Marie taught me most of my technical skills and how to keep my samples organized. Elaine, Zhimin, and Alexandra helped lighten my load on hard days and kept me cheerful. My committee members have always been extremely supportive of my work, even when I sheepishly reported that I had to abandon a large line of experiments, and have provided valuable insight in project development. I have benefitted from the guidance of numerous mentors at the University of Virginia, for which I am extremely grateful. My mom, dad, Anne, brother Alden, and sister-in-law Shauna have been there to remind me that people and family are at the focus of everything I do. 


\section{List of Figures}

\section{Chapter 1}

Figure 1.1: Mammalian iron distribution .................................................

Figure 1.2: Effect of hepcidin on iron distribution.....................................6

Figure 1.3. Mechanisms of hepcidin induction and inhibition........................10

Figure 1.4: Growth cycle of pathogenic Aspergillus species..........................28

Figure 1.5: Siderophore synthesis in Aspergillus species...........................32

Chapter 2

Figure 2.1. Iron availability during Klebsiella pneumonia..........................43

Figure 2.S1. Lung iron content in wildtype mice treated with PBS or iron citrate.45

Figure 2.2. Hepcidin induction during Klebsiella pneumonia.........................48

Figure 2.3: The role of IL-6 in hepcidin induction during Klebsiella pneumonia...50

Figure 2.4: The role of hepcidin in Klebsiella pneumonia and dissemination......54

Figure 2.S2: Aspartate transaminase (AST) and alanine transaminase (ALT) in wildtype and hepcidin $/-$ mice...........................................56

Figure 2.S3. Iron-depleted hepcidin-/- mice are not susceptible to infection compared to wildtype mice.................................................

Figure 2.5. Effect of iron on Klebsiella growth in vitro and in vivo................62

Figure 2.6. Leukocyte responses during Klebsiella pneumonia.......................65

Figure 2.S4. Reactive oxygen species (ROS) production in infected mice..........67

Figure 2.7: The effect of hepcidin agonist treatment in Klebsiella pneumonia in

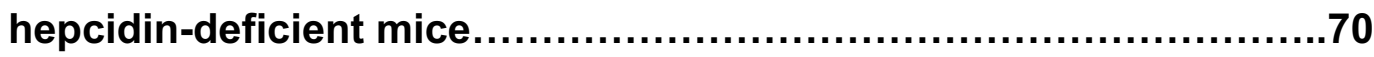

Figure 2.8: The effect of hepcidin agonist treatment in Klebsiella pneumonia in 
wildtype mice.

Chapter 3

Figure 3.1. Pulmonary hemorrhage during invasive pulmonary aspergillosis......89

Figure 3.2. Uptake of heme and tin PPIX in vitro....................................92

Figure 3.3. Accumulation and utilization of heme in vitro. ...........................98

Figure 3.4. The effect of exogenous hemin on invasive pulmonary aspergillosis.

Figure 3.5. Method comparison for fungal burden assay. 102

Figure 3.6. Markers of acute lung injury in mice challenged with dead $A$.

fumigatus germlings

Chapter 4

Figure 4.1. Proposed role of hepcidin during Gram-negative pneumonia........114

Figure 4.2. Does hepcidin dysregulation contribute to infection during cirrhosis?

Figure 4.3: Evidence of hyperferremia following infection during hepcidin deficiency 128 


\section{List of Tables}

Table 4.1. Experimental groups to test the role of dysregulated iron metabolism

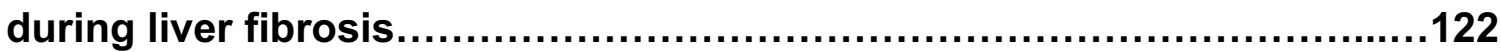

Table 4.2: Experimental groups to test the hypothesis that hepcidin mimetics can be used against clinical isolates. 124 


\section{Abbreviations}

DMT1 Divalent metal transporter 1

Tfr2 Transferrin receptor 2

HFE Human hemochromatosis protein

BMPR Bone morphogenetic protein receptor

STAT Signal transducer and activator of transcription

Fpn1 Ferroportin

iNOS Inducible nitric oxide synthase

IFN Interferon

TLR Toll-like receptor

CLP Cecal ligation and puncture

$\mathrm{HCV} \quad$ Hepatitis $\mathrm{C}$ virus

$\mathrm{C} / \mathrm{EBP} \alpha \quad \mathrm{CCAAT} /$ enhancer-binding protein alpha

ROS Reactive oxygen species

CGD Chronic granulomatous disease

FusC Fusaranine C

TAFC Triacetyl fusaranine $\mathrm{C}$

FC Ferricrocin

HFC Hydroxyferricrocin

BAL Bronchoalveolar lavage

AST Aspartate aminotransferase

ALT Alanine aminotransferase

WT Wildtype 
SOCS Suppression of cytokine signaling

CFU Colony forming unit

EDTA Ethylenediaminetetraacetic acid

UIBC Unsaturated iron binding capacity

TIBC Total iron binding capacity

LPI Labile plasma iron

DFP Deferriprone

DCDFA 2',7'-dichlorofluorescin diacetate

TBHP tert-Butyl hydroperoxide

HBSS Hanks balanced salt solution

SEM Standard error of the mean

GMS Grocott's methenamine silver stain

AMM Aspergillus minimal media

PPIX Protoporphyrin

ANOVA Analysis of variance

TNF Tumor necrosis factor

PAMP Pathogen associated molecular pattern

SDA Sabouraud dextrose agar

UVA University of Virginia 


\section{Chapter I: Introduction}

Published in part: Michels, K., Nemeth, E., Ganz, T. \& Mehrad, B. Hepcidin and host defense against infectious diseases. PLOS Pathog 11, e1004998 (2015).

\subsection{Iron metabolism in mammals}

Iron is essential to the survival of virtually all known organisms because it is necessary for the function of many proteins, such as hemoglobin, myoglobin, and enzymes involved in oxidative phosphorylation. However, ionic iron is highly toxic due to its reactivity with oxygen in the so-called Fenton reaction, which generates the hydroxyl radical and other toxic reactive oxygen species. As an element that is both essential and dangerous, iron is tightly associated with proteins in biological systems, including those involved in iron transport and storage, and free iron ions are present only in extremely low concentrations ${ }^{1}$. In multicellular organisms, the low availability of iron imposes a severe nutritional restriction on invading microbes, and hosts have evolved methods to further limit iron availability in the context of infection. Professional pathogens, on the other hand, have evolved elegant strategies to scavenge iron from the iron-binding proteins of the hosts. Multiple lines of evidence suggest that this battle for iron is a critical component of antimicrobial defenses in many infectious diseases ${ }^{2}$.

The global distribution of iron in mammalian hosts is depicted in Figure 1.1. Iron is absorbed from the diet in duodenal enterocytes via divalent metal transporter 1 (DMT1). Extracellular iron, which constitutes a small proportion of total body iron, is transported in the blood in association with the protein transferrin. Transferrin has an extremely high affinity for ferric iron $\left(10^{20} \mathrm{M}^{-1}\right)$ and at baseline, only $15-50 \%$ of transferrin sites are occupied with iron; thus, the appearance of non-transferrin bound iron ions 
appear only in the setting of iron overload and disease ${ }^{3}$. Although representing a very small portion of total body iron, plasma iron is subject to rapid turnover (half-life of about one hour) due to continuous utilization by other cells in the body, primarily erythroid precursors, which import iron via the transferrin receptor ${ }^{4}$. The majority $(\sim 70 \%)$ of the iron in the body is found in circulating erythrocytes packaged as heme in hemoglobin. Splenic macrophages engulf senescent erythrocytes, oxidize the scavenged heme to recover iron, and release iron back into circulation. This iron recycling pathway is the central hub of iron utilization and accounts for the vast majority of daily iron metabolism, and comparatively little iron is absorbed from the diet on a daily basis ${ }^{1}$.

Hepatocytes and Kupffer cells serve as an important reservoir that stores or releases iron to maintain homeostasis, thus acting as a buffer to ensure that enough iron is released for daily needs, while also limiting overt toxicity. Other organs import comparatively small amounts of iron as needed. Stores of intracellular iron are maintained in association with the protein ferritin, which functions as a hollow storage vault that can contain up to 4500 ferric iron ions ${ }^{5}$. Iron is lost from the body by entirely passive processes, such as shedding of enterocytes or bleeding, and mammals have no controlled way of eliminating excess iron ${ }^{1}$. 


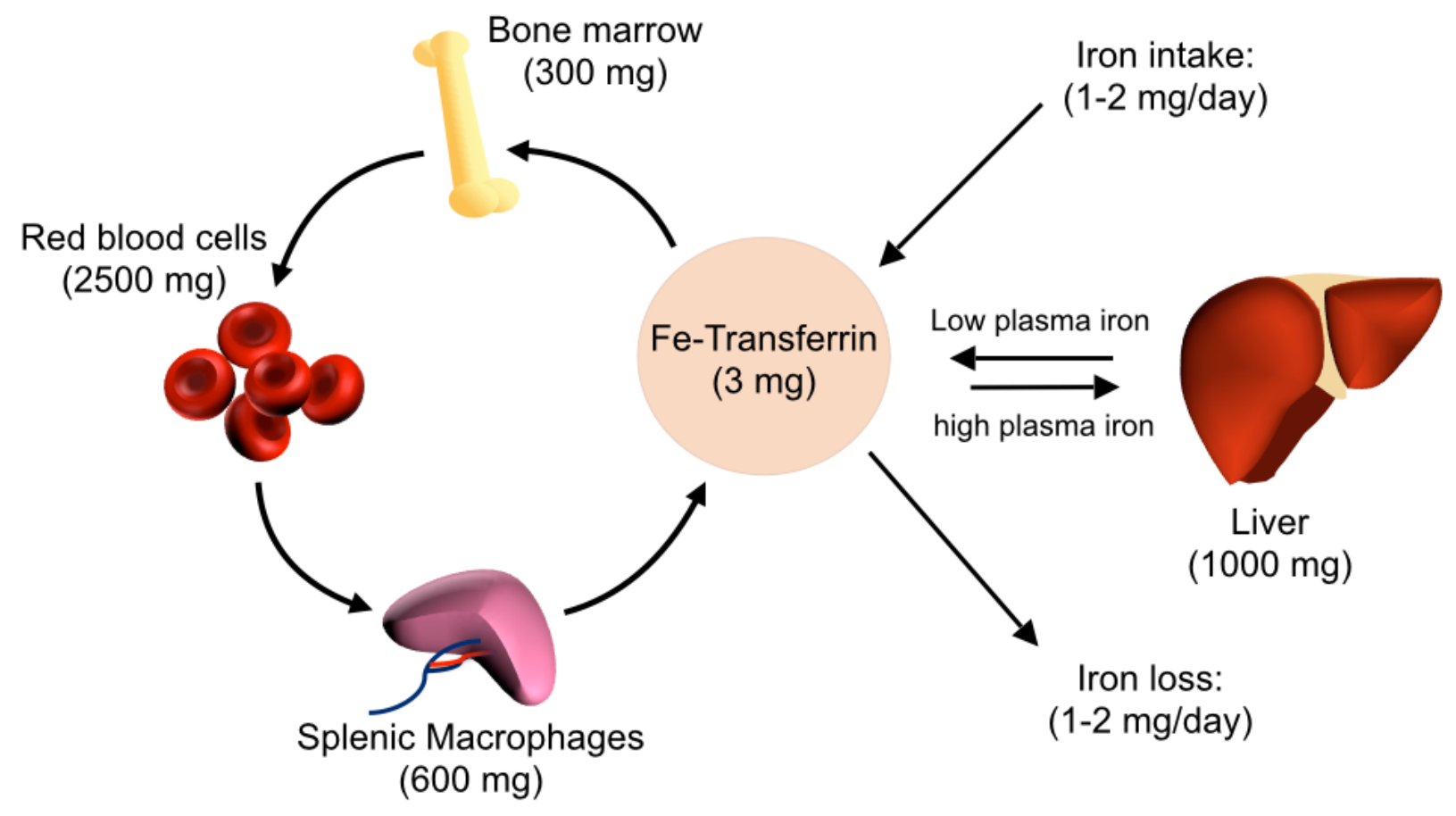


Figure 1.1. Mammalian iron distribution. Iron is absorbed from the diet by duodenal enterocytes and transported into the bloodstream, where it is bound by transferrin. Most iron is incorporated into erythrocytes for heme synthesis. Splenic macrophages recover iron from senescent erythrocytes and release iron into circulation via ferroportin. Smaller amounts of iron are imported into other tissues as needed. Iron loss is not directly regulated and occurs through minor bleeding and shedding of duodenal enterocytes. Approximate iron content of adult human tissues is represented in parentheses. 


\subsection{The role of hepcidin in iron metabolism}

The peptide hepcidin is the master regulator of iron homeostasis in vertebrates ${ }^{6-}$

8. Hepcidin was first described as a cationic antimicrobial peptide with microbicidal properties against many microorganisms in vitro ${ }^{7}$. Hepcidin is strongly induced during inflammation ${ }^{9}$ and emerging data support its role in the pathogenesis of a number of infections. Here, I will discuss hepcidin in the context of resistance and susceptibility to infectious diseases.

The hepcidin-ferroportin axis controls extracellular iron concentrations and total body iron levels. Ferroportin is a membrane protein that is the major exporter of iron from mammalian cells, including macrophages that recycle iron from old cells, duodenal enterocytes that absorb iron from diet, and hepatocytes that store iron. Hepcidin limits the pool of extracellular iron by binding ferroportin and mediating its degradation, thus preventing iron release from intracellular sources (Figure 1.2 $)^{10}$. Sustained elevations of hepcidin result in insufficient iron availability for erythropoiesis, causing an ironrestricted anemia. In contrast, the inability to produce or respond to hepcidin causes hereditary hemochromatosis, a group of inherited iron overload disorders resulting from excessive dietary iron absorption and the inability to sequester iron in macrophages ${ }^{11}$. 
Low hepcidin conditions:

Iron exported via ferroportin

into extracellular space

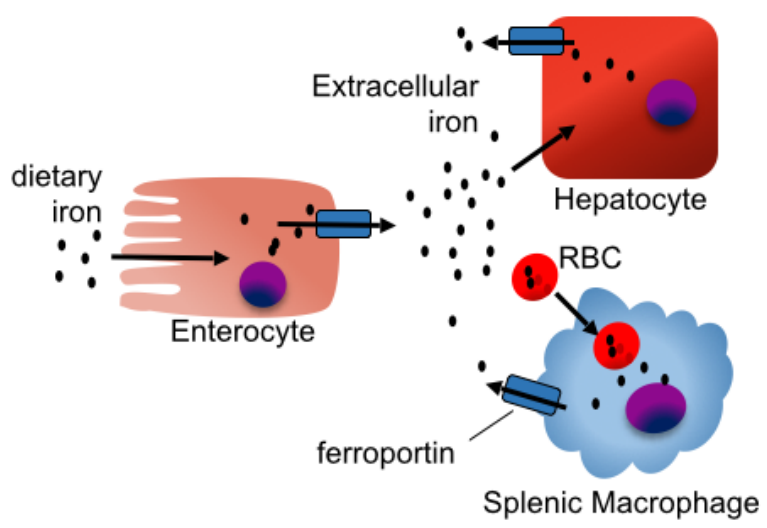

High hepcidin conditions:

Ferroportin degraded, iron

accumulates intracellularly

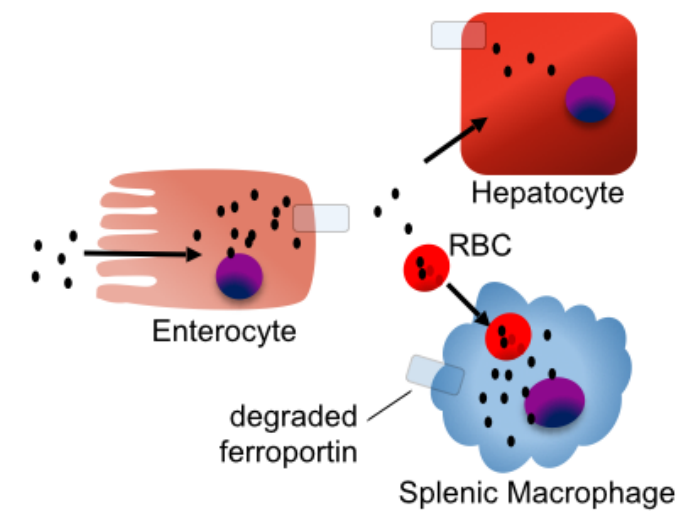


Figure 1.2. Effect of hepcidin on iron distribution. In the absence of hepcidin, iron absorbed from the diet by duodenal enterocytes is transported into the serum via ferroportin, and iron captured from senescent red blood cells is exported from splenic macrophages. In the presence of hepcidin, iron is retained in duodenal enterocytes, which eventually shed from the intestinal tract, blocking iron absorption from the diet. Mononuclear phagocytes retain and accumulate recycled iron rather than releasing it back into circulation, causing a rapid drop in serum iron levels. 
Most circulating hepcidin is produced by hepatocytes. Hepatocyte-specific deletion of hepcidin recapitulates the hemochromatosis phenotype of global hepcidindeficient animals, indicating that hepatocyte-derived hepcidin is necessary for iron homeostasis in steady state $^{12}$. Hepatocyte hepcidin expression is stimulated by elevated extracellular or stored iron and, independently, also by inflammatory stimuli. Conversely, hepcidin expression is inhibited by hypoxia and erythropoiesis ${ }^{13,14}$. Regulation of hepcidin by iron depends on the hemojuvelin/BMP receptor complex activating the SMAD1/5/8 and SMAD4 signaling pathway (Figure 1.3) $)^{15,16}$.

During inflammatory states, hepcidin expression is induced as part of the acute phase response by the cytokine IL-6 via the JAK-STAT3 pathway, although this pathway is insufficient to induce hepcidin without BMPR signaling ${ }^{17,18}$. Recently, it was shown that Activin B also upregulates hepcidin as part of the acute phase response via BMP receptors ${ }^{19}$. In animal models, diverse inflammatory and infectious stimuli, including Streptococcus pneumoniae, Pseudomonas aeruginosa, Aspergillus fumigatus, influenza, turpentine and LPS robustly induce hepcidin in the liver via the induction of IL-6, resulting in rapid reduction in serum iron levels ${ }^{20-22}$. Sustained elevation of hepcidin expression, as occurs in many inflammatory states, results in anemia due to reduced availability of iron for hematopoiesis, a condition previously known as "anemia of chronic disease" and more accurately renamed as "anemia of inflammation"23.

In addition to hepatocytes, low levels of hepcidin are expressed by many cell types, including myeloid leukocytes ${ }^{24}$. Phagocyte hepcidin expression can be induced via autocrine and paracrine production of IL-6, and at least in vitro, also by direct engagement of pathogen recognition receptors ${ }^{25,26}$. The contribution of leukocyte- 
derived hepcidin has not been formally examined but is hypothesized to contribute to host defense by autocrine and paracrine effects ${ }^{27,28}$. In addition, while hepcidin is directly microbicidal in micromolar concentrations in vitro ${ }^{7}$, it is unknown if local tissue hepcidin levels reach sufficient concentrations to exert microbial killing in vivo. 


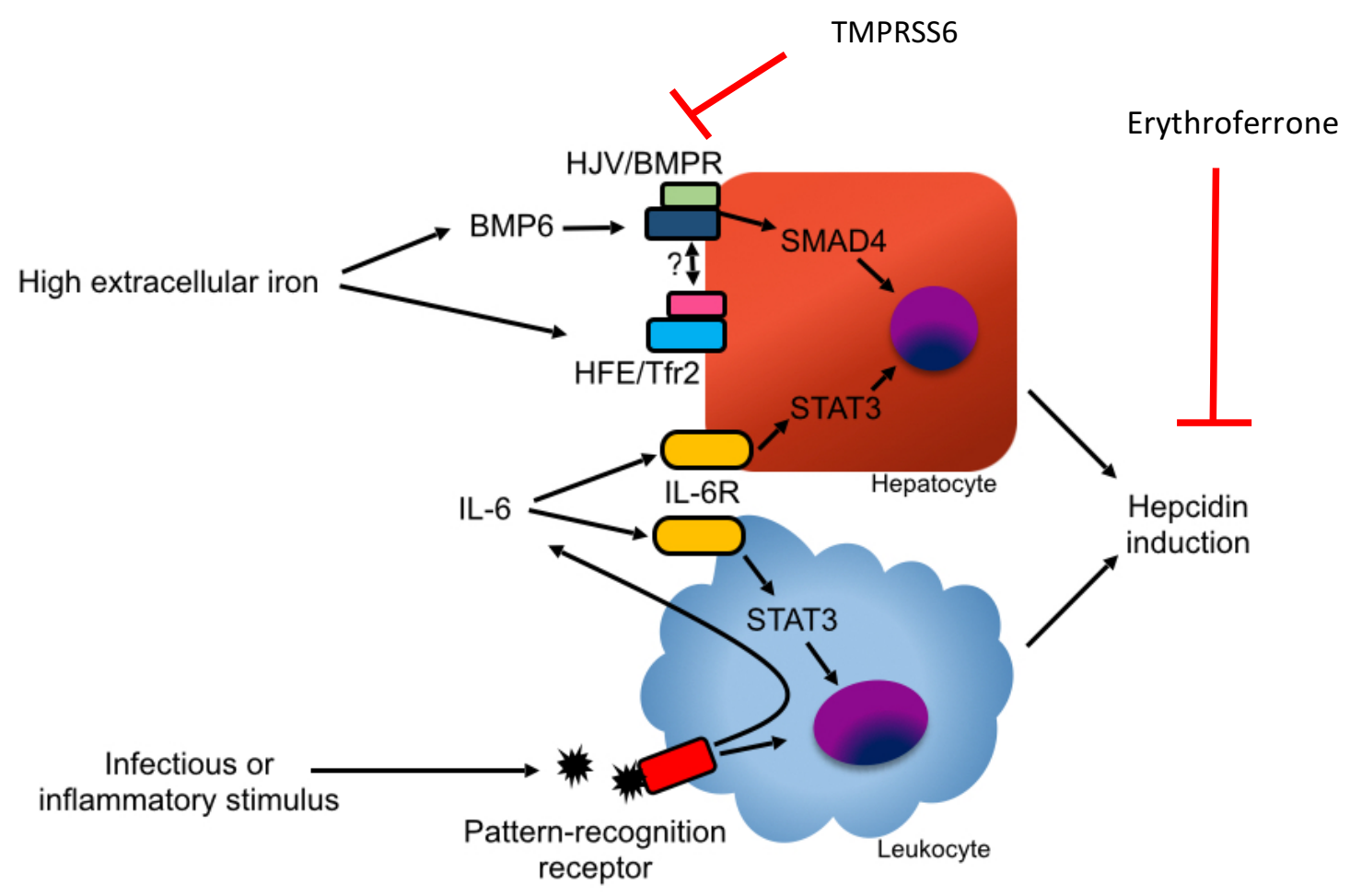


Figure 1.3. Mechanisms of hepcidin induction and inhibition. In hepatocytes, hepcidin induction is mediated primarily by BMP6 binding with the HJV/BMPR complex. BMP6 is induced by high iron levels in both parenchymal and non-parenchymal cells in the liver via an undefined mechanism, and is critically important for maintaining normal iron homeostasis ${ }^{16,29}$. High holo-transferrin levels stabilize the transferrin receptor 2 (Tfr2)/HFE complex, which promotes hepcidin induction. This complex appears to require the HJV/BMPR complex to function, although there are conflicting models to explain this interaction ${ }^{30-32}$. Hepcidin can also be induced by IL-6 via STAT3 signaling in hepatocytes and myeloid leukocytes. Inflammation can stimulate hepcidin production in myeloid leukocytes through pathogen recognition receptor signaling and through autocrine and paracrine production of IL-6. The transmembrane serine protease matriptase-2 (TMPRSS6), a highly conserved protein synthesized in the liver, inhibits hepcidin production by degrading HJV in response to low iron levels and is essential for normal iron homeostasis ${ }^{33}$. Erythroferrone, a hormone produced during erythropoiesis, inhibits hepcidin by an unclear mechanism and is important for suppressing hepcidin during anemia ${ }^{14,34}$. 


\subsection{The role of hepcidin in specific infections}

\subsubsection{Intracellular infections}

Hepcidin results in the accumulation of iron within cells, particularly cells of the mononuclear phagocyte system, potentially benefiting pathogens that occupy this intracellular niche. Type I hemochromatosis, the most common form of hereditary hemochromatosis, is caused by loss-of-function mutations in the HFE gene. HFE protein mediates hepcidin expression in response to increased extracellular iron, and in the absence of is results in attenuated hepcidin expression, increased iron absorption, increased extracellular iron, and reduced iron within macrophages. HFE mutations have arisen independently in several human populations, and the most common mutation, $\mathrm{C} 282 \mathrm{Y}$, has a heterozygous prevalence of up to $10 \%$ in northern European populations ${ }^{35}$. Although difficult to test, it is hypothesized that HFE mutations became prevalent by conferring a survival benefit during population bottlenecks caused by hostadapted pathogens that reside within macrophages as part of their life-cycle ${ }^{36}$.

Salmonellosis: Although in vitro data generally support the role of hepcidin in promoting Salmonella growth in macrophages, in vivo studies have provided conflicting results. Experimental Salmonella infection of macrophages in vitro has been shown to induce hepcidin expression via autocrine or paracrine mechanisms, causing intracellular iron accumulation and allowing for greater bacterial growth. In vitro infection of murine macrophages with Salmonella spp. resulted in lower intracellular bacterial burdens when host cells were transfected to overexpress ferroportin ${ }^{37,38}$. In addition, hepcidin treatment increased bacterial burden in cells expressing WT, but not hepcidin-resistant, ferroportin by presumably trapping iron inside of cells ${ }^{37,39}$. Similar results have been 
published with macrophages infected with Chlamydia and Legionella species ${ }^{40}$. Similarly, elicited peritoneal macrophages from Hfe-/- mice had lower iron content and, when infected with $S$. enterica in vitro, had lower bacterial content compared to wildtype cells ${ }^{38}$.

In vivo, mice develop increased hepcidin levels and hypoferremia after oral infection with S. typhimurium ${ }^{41}$. This hepcidin induction was proposed to be detrimental to the host because prevention of hepcidin induction by an inverse agonist of estrogenrelated receptor gamma was associated with improved mouse survival after $S$. typhimurium infection. In a different study, however, opposite results were reported: Intravenous infection with S. typhimurium did not increase hepcidin mRNA levels in the liver, and hepcidin-deficient mice were more susceptible to infection than their wild-type counterparts $^{42}$. The literature on the susceptibility of Hfe-deficient mice to salmonellosis is similarly contradictory. Hfe-deficient mice have been reported to have attenuated intestinal inflammation but higher fecal and systemic bacterial burdens after oral infection in studies that used streptomycin-pretreated mice ${ }^{43}$. In other studies, Hfedeficient mice were reported to have reduced death and bacterial burdens after oral and intraperitoneal inoculation of $S$. typhimurium and this was ascribed to the greater production of lipocalin-2 in Hfe-/- macrophages ${ }^{38}$.

The reasons for the discrepancies in the in vivo studies of S. typhimurium are unknown, but may relate to factors such as the age and diet of the experimental animals, which could influence the extent of iron overload at the time of infection and possibly alter the pathogenicity of Salmonella. Furthermore, extrapolation of data from Hfe-deficient animals to the role of hepcidin is not straightforward, since hepcidin 
expression in Hfe-deficient mice is not abrogated but only attenuated relative to degree of iron overload ${ }^{44}$. In addition, there are conflicting reports as to whether Hfe influences hepcidin induction to inflammatory stimuli ${ }^{44-46}$.

Mycobacterial infections: African iron overload, a condition caused by a combination of high dietary iron intake and non-HFE hereditary hemochromatosis, is strongly associated with death from tuberculosis ${ }^{47,48}$, potentially implicating hepcidin in host defense against mycobacteria. Consistent with this, ferroportin over-expressing macrophages have lower mycobacterial burden as compared to normal macrophages when infected with $M$. tuberculosis in vitro ${ }^{49}$. Ferroportin overexpressing macrophages also have reduced iNOS production and phagocytic ability, suggesting that depletion of intracellular iron stores may interfere with macrophage effector functions ${ }^{49}$. Exposure of macrophages to mycobacteria and IFN- $\gamma$ synergistically induce the expression of hepcidin in vitro, and macrophage-derived hepcidin co-localizes with M. tuberculosis in co-incubation studies ${ }^{28,50}$ but the relevance of macrophage-derived hepcidin to in vivo host defense in unclear. Similarly, high concentrations of hepcidin have been shown to inhibit $M$. tuberculosis growth in vitro ${ }^{28}$ but it is unknown whether these concentrations are relevant to in vivo infections. Macrophages from humans with type I hemochromatosis have lower iron accumulation and lower bacterial burden when infected with M. tuberculosis as compared to cells from healthy donors; conversely, mycobacteria acquire iron more efficiently from macrophages of healthy subjects as compared to cells from patients with hemochromatosis ${ }^{50,51}$. These data suggest that hepcidin-mediated increases in intracellular iron may be harmful to the host during mycobacterial infections, but this hypothesis has not been addressed directly in vivo. In 
another study, Hfe-deficient mice with iron overload had increased tissue bacterial burden after intravenous infection with $M$. avium compared to wildtype animals ${ }^{52}$, an unexpected result for animals predicted to have iron-depleted macrophages. As with studies in salmonellosis, however, it is difficult to extrapolate data from the HFE mouse model to the role of hepcidin during the infection.

Leishmaniasis: While limited, the current literature suggests that hepcidin induction is detrimental to host defense against leishmaniasis. An in vitro study showed that murine bone marrow-derived macrophages up-regulate hepcidin in a TLR4-dependent manner, reduce cell surface ferroportin protein and accumulate intracellular iron following infection with $L$. amazonensis. Macrophages from hepcidin-deficient mice had lower intracellular burden of parasites as compared to wildtype macrophages after in vitro infection, and hepcidin treatment of wildtype macrophages results in higher parasitic burdens ${ }^{53}$.

\subsubsection{Extracellular infections}

Individuals with hereditary hemochromatosis are notably susceptible to sepsis caused by specific pathogens whose pathogenicity is augmented in the presence of free iron. These so-called siderophilic bacteria include some Vibrio and Yersinia species, with less conclusive evidence available for other Gram-negative bacteria including Salmonella and Escherichia species ${ }^{11}$. Susceptibility to siderophilic infections in hemochromatosis is thought to be mediated by increased availability of extracellular iron, but in observational studies it is difficult to exclude the contribution of other mechanisms, such as possible immune dysfunction as a result of iron overload ${ }^{54}$. 
Vibrio infection: Wild-type mice rapidly up-regulate hepcidin and develop hypoferremia after subcutaneous infection with $V$. vulnificus, whereas hepcidin-deficient mice exhibited only mild hypoferremia and are much more susceptible to the infection ${ }^{55}$. The susceptibility of hepcidin-deficient mice was associated with high blood and tissue burden of $V$. vulnificus and was specifically attributable to high levels of extra-cellular iron, since hepcidin-deficient mice with low iron stores (but high serum iron) were also susceptible to infection. Treatment of iron-overloaded hepcidin knockout mice with a hepcidin agonist was sufficient to restore hypoferremia (without changing iron stores) and to protect hepcidin-deficient mice from infection. Ex vivo studies of bacterial growth in sera from treated animals showed that hepcidin agonists prevented the growth of $V$. vulnificus by restricting iron availability rather than by direct antimicrobial activity. Hepcidin agonists did not provide any further protection from $V$. vulnificus infection in wild-type mice, again arguing against a direct microbicidal effect of these peptides.

Sepsis syndrome: Whether hepcidin plays any role in modulating the course of sepsis is unknown, although several mouse studies suggested a protective effect. In a murine cecal ligation and puncture (CLP) model of polymicrobial intra-abdominal sepsis, mice treated intravenously with an adenovirus carrying anti-hepcidin shRNA had higher mortality and bacterial burden associated with increased serum iron levels, whereas conditioning mice on a low-iron diet or treating with an iron chelator resulted in protection following anti-hepcidin shRNA treatment ${ }^{56}$. Similarly, mice that received inhaled anti-hepcidin shRNA adenovirus had higher mortality and more severe lung injury after CLP compared to mice that received control adenovirus ${ }^{39}$. These studies 
suggested that expression of hepcidin may be protective during sepsis caused by resident gut flora.

\subsubsection{Malaria}

In human studies, iron supplementation is associated with increased incidence and severity of malaria in some ${ }^{57}$ but not all ${ }^{58}$ studies, whereas dietary iron deficiency is associated with reduced malaria parasitemia and death ${ }^{59}$. Iron deficiency also promotes protection against infection with Plasmodium berghei in mice ${ }^{60,61}$, suggesting a role for hepcidin in this infection. Human and mice infected with malaria have elevated plasma hepcidin levels which correlate positively with parasitemia and plasma IL-6 ${ }^{62-65}$. Human P. falciparum patients with severe anemia have reduced hepcidin levels, possibly a result of erythroferrone-mediated hepcidin suppression ${ }^{34,62}$. In vitro, P. falciparuminfected erythrocytes induce hepcidin mRNA expression in human peripheral blood monocytes and monocyte-derived macrophages in an IL-6 independent but IL-10 dependent manner ${ }^{66,67}$, but the relevance of leukocyte-derived hepcidin during infection is undefined.

Hepcidin has complex effects in malaria. On one hand, by causing iron restriction, elevated hepcidin likely contributes to anemia. On the other hand, hepcidin may have protective effects in mice during experimental malaria ${ }^{64,65}$. Immunoneutralization of hepcidin results in increased parasitemia and death in $P$. berghei infection, whereas pretreatment of animals with a hepcidin-carrying lentivirus construct protected against parasitemia and death as compared to mice treated with a control lentivirus ${ }^{65}$. Hepcidin also appears to be a host defense mechanism that protects hosts with parasitemia against a second malaria infection ${ }^{64}$; such super-infections are 
associated with increased mortality in endemic areas. Blood-stage infection induced liver hepcidin expression that was, as expected, associated with increased splenic and decreased hepatic iron content following infection. Transgenic over-expression or administration of hepcidin to infected mice provided protection by reducing the burden of the parasites in the liver. These data suggest that hepcidin protects against malaria by reducing iron availability to parasites.

\subsubsection{Viral infections}

While the induction of serum hepcidin has been documented during several human and murine viral infections ${ }^{20,25}$, relatively little is known about the contribution of hepcidin to the pathogenesis of most viral infections. In an interesting study of serial samples, plasma hepcidin induction during early HIV infection correlated with subsequent viral load set point ${ }^{68}$, although the mechanism underlying this correlation is unclear.

Hepatitis C: Hepatic iron accumulation is common in hepatitis C virus (HCV) infection and contributes both to liver fibrosis and increased the risk of hepatocellular carcinoma $^{69}$. Patients with hepatitis $\mathrm{C}$ who have HFE mutations are further predisposed to hepatic iron overload and worse liver fibrosis ${ }^{70}$. Although circulating hepcidin levels correlate positively to the degree of iron overload in chronic HCV infection, patients with HCV infection have a relative deficiency of hepcidin: compared to uninfected controls, HCV patients had lower hepcidin levels for given serum ferritin level, suggesting that hepcidin expression may be blunted during HCV infection ${ }^{71-73}$. Hepcidin was not induced even during acute phases of $\mathrm{HCV}$ infection ${ }^{68}$, unlike most other infections. 
Consistent with this, in vitro infection of a hepatocellular carcinoma cell line with HCV resulted in suppression of hepcidin transcription that was associated with higher ROS production $^{74}$. This effect was attributable to reduced binding of the transcription factor CCAAT/enhancer-binding protein alpha (C/EBPa) to the hepcidin promoter, and both binding to and expression of hepcidin could be restored by treatment with antioxidants ${ }^{74}$. In the context of animal models, transgenic mice expressing HCV core protein develop increased serum and hepatic iron, but have reduced splenic iron over time as compared to wild-type control animals ${ }^{75}$. This phenomenon was associated with suppressed hepcidin expression and higher ferroportin protein in liver, spleen and duodenum. Primary hepatocytes from transgenic mice that were transfected with a hepcidin promoter/luciferase reporter construct showed lower luciferase activity compared to hepatocytes cultured from control mice, again associated with reduced binding of C/EBPa to the hepcidin promoter and increased levels of ROS species ${ }^{75}$. Taken together, these experiments suggest that HCV-mediated hepcidin suppression contributes to iron overload and disease pathology in HCV infection, although whether decreased hepcidin levels and consequent tissue iron loading play any role in viral replication is still unknown.

\subsubsection{Iron-independent functions of hepcidin}

Whether any iron-independent functions of hepcidin have pathophysiological relevance remains to be demonstrated, but some studies have proposed that hepcidin can influence immune responses independent of its role in iron homeostasis. One study proposed that hepcidin regulates production of cytokines and dampens inflammatory 
responses via the activation of the Jak2 pathway ${ }^{76}$, as Jak2 is recruited to ferroportin after hepcidin binding. However, it was subsequently shown that Jak2 is not activated by hepcidin and does not interact with ferroportin ${ }^{77}$, challenging the proposed mechanism.

A different study showed that iron-deficient macrophages had a more pronounced inflammatory response to LPS treatment, and the inflammatory responses could be dampened with hepcidin treatment alone ${ }^{78}$. To confirm the role of hepcidin, mice deficient in the serine protease TMPRSS6 were used as these animals have iron deficiency due to high constitutive expression of hepcidin ${ }^{33}$. Tmprss6-/- mice demonstrated a blunted inflammatory response to LPS despite their iron-deficient status $^{78,79}$. This suggested that hepcidin may modulate inflammatory responses independently of iron, although more comprehensive studies that evaluate intracellular iron levels are needed. However, other studies report opposite results: iron-depleted macrophages from Hfe-/- mice (which also have low hepcidin) had attenuated inflammatory response to LPS and Salmonella ${ }^{43}$. Thus, more extensive evaluation of hepcidin effects on inflammation is needed.

\section{Conclusions}

Hepcidin is potently increased by inflammation, but the role of hepcidin in innate immunity is only beginning to be understood. Hepcidin restricts access to extracellular iron, and this form of "nutritional immunity" is important in at least some extracellular bacterial infections. In contrast, hepcidin induces iron accumulation in macrophages and may be detrimental in defense against pathogens that occupy this intracellular niche. This effect has been demonstrated convincingly in vitro but is not supported by in vivo 
data $^{28,37,40,50}$. Interrogating the role of hepcidin in animal models of intracellular infections should further clarify the complex relationship between iron distribution and pathogenesis of such infections in humans.

In the absence of in vivo data, any iron-independent role of hepcidin in host defense remains speculative. In particular, there is little evidence to support a direct microbicidal role for hepcidin in mammalian infections. The current literature suggests that hepcidin may dampen inflammatory cytokines through a mechanism that is not well understood. As excessive inflammation is damaging in many infections, the potential role of hepcidin as a mediator of the innate immune response is a new and unexpected area of study.

The role of hepcidin remains undefined in most infections and awaits further investigation. For example, although hepcidin is induced in response to several viral and

fungal pathogens ${ }^{21,80}$, its contribution to host defenses against these infections is largely unknown. With the exception of malaria and Leishmaniasis, hepcidin has not been investigated in parasitic infections.

\subsection{The role of iron during Klebsiella pneumoniae infections}

\subsubsection{Pathogenesis and host defense against Klebsiella pneumonia}

Gram-negative pneumonia is a common and dangerous illness that is becoming increasingly difficult to treat due to the rapid rise of antibiotic resistance in the infective agents. Klebsiella pneumoniae is a member of the Enterobacteriaceae family and is a minor component of the gut flora in humans where it does not cause disease; however, if $K$. pneumoniae is aspirated into the lungs, it can cause a severe and dangerous pneumonia ${ }^{81}$. Klebsiella pneumoniae is a major cause of nosocomial pneumonia, which 
typically afflicts patients already in poor health, and can display high levels of antibiotic resistance.

As an opportunistic pathogen, K. pneumoniae lacks many traditional virulence factors. It does not produce any known toxins or secretion systems that promote disease pathogenesis in the lung. Encapsulation is considered the most important virulence determinant in $K$. pneumoniae pneumonia and bacteremic infections ${ }^{81}$. Virtually all clinical isolates produce a capsule, and capsule production is required for virulence in murine infection models ${ }^{82}$. A hypermucoviscous phenotype is also associated with the ability of certain strains of $K$. pneumonae to cause severe disease in otherwise healthy patients, although it is unclear if capsule hyper production is important for enhanced virulence in the emergent strains ${ }^{83}$. Fimbriae do not seem to play a role during pneumonia, as type 1 and type 3 fimbriae are not expressed during pulmonary infections, although type 1 fimbriae appear to be important for urinary tract infections $^{84,85}$. Siderophores and iron uptake systems are important in $K$. pneumoniae infections, and are discussed in greater detail below.

Host defense against $K$. pneumoniae during experimental murine infection is mediated by a robust, inflammatory innate immune response. Early production of IL-17, IL-22, IL-23, KC, GM-CSF, and TNF are required for host defense against infection ${ }^{86-88}$. Early reports attributed the early production of IL-17 and IL-22 during K. pneumoniae pneumonia to $T_{h} 17$ cells; however, it is more likely that innate lymphoid cells or $\gamma \delta \mathrm{T}$ cells are responsible for early production of these cytokines, and it is not clear if the adaptive immune system plays an important role in murine infection ${ }^{89}$. Defects in neutrophil trafficking or function renders mice highly susceptible to infection ${ }^{90,91}$. More 
recently, our lab has shown that alveolar macrophage survival and CCR2-dependent monocyte recruitment is also protective against infection ${ }^{92,93}$.

\subsubsection{Role of siderophores and iron in K. pneumoniae infections}

Siderophores are low molecular weight iron chelators produced by a wide variety of microbes that scavenge iron from the environment. As with many other pathogens, siderophores are recognized as virulence factors in $K$. pneumoniae ${ }^{94}$. K. pneumoniae can produce up to four siderophores, which are shared by other pathogens in the Enterobacteriaceae family, that appear to have overlapping and complementary roles in virulence. Enterobactin (also known as enterochelin), encoded by EntB, is a catechol siderophore which has the highest affinity for iron of any known compound and is expressed in virtually all pathogenic strains of $K$. pneumoniae ${ }^{95-97}$. Enterobactin is neutralized and sequestered by the host-derived protein, lipocalin-2. Of the $K$. pneumoniae strains that can produce enterobactin, a subset is able to produce glycosylated enterobactin (also known as salmochelin), encoded by the IroA locus, which permits evasion of lipcalin- $2^{98}$. About $50 \%$ of $K$. pneumoniae clinical isolates are able to produce aerobactin, a hydroxamate siderophore found in several pathogenic Enterobactericeae with a lower affinity for iron than enterobactin ${ }^{95}$. An additional 10$20 \%$ of $K$. pneumoniae clinical isolates are able to produce yersiniabactin, a phenolate siderophore with an iron affinity between that of aerobactin and enterobactin ${ }^{95}$.

The relative contribution of each siderophore to virulence seems to depend on the strain of $K$. pneumoniae and the iron status of the host. Enterobactin is ubiquitously expressed and required for efficient growth of $K$. pneumoniae in serum ${ }^{99}$, while 
glycosylated enterobactin is required for $K$. pneumoniae growth in serum in the presence of lipocalin- $2^{99}$. In a murine infection model, lipocalin-2 knockout mice were susceptible to colonization of the nasopharynx, pneumonia, and were extremely sensitive to disseminated disease caused by $K$. pneumoniae strains that used enterobactin as the sole siderophore receptor ${ }^{97,99,100}$.

Aerobactin is also recognized an importance virulence factor in pathogenic strains. In one study, aerobactin production distinguished high-virulence strains from low-virulence strains of $K$. pneumoniae in an intraperitoneal mouse model of infection ${ }^{96}$. In a separate study of a hypermucosviscous $K$. pneumoniae strain that could produce all four siderophores, aerobactin was found to be the only siderophore necessary and sufficient for virulence ${ }^{101}$. Consistent with this finding, the same group found that In a subcutaneous and pneumonia model of infection in outbred mice, a K. pneumoniae mutant deficient in aerobactin had severely impaired virulence compared to the parent strain even with the other siderophore systems intact ${ }^{102}$. The authors posit that the aerobactin locus is the most active site of siderophore synthesis in the strain studied.

There are several lines of evidence that yersiniabactin promotes bacterial growth in the nasopharynx and airways, but plays little role in disseminated disease. First, one study found that yersiniabactin production is overrepresented in $K$. pneumoniae isolates found in the airways as compared to other isolates ${ }^{97}$. The same group found that mutants possessing yersiniabactin as the sole siderophore were able to effectively colonize the nasopharynx and cause pneumonia in the lung, but were unable to cause disseminated disease ${ }^{97,99}$. Consistent with this, another group found that $K$. pneumoniae 
deficient for yersiniabactin production grew poorly in the lung compared to the parent strain, or to the isogenic strain deficient in enterobactin ${ }^{103}$.

Host iron overload has also been identified as a susceptibility factor to $K$. pneumoniae infections in experimental animal infections. One study found that during an intraperitoneal mouse infection, avirulent strains of $K$. pneumoniae could cause lethal disease if animals were treated with iron citrate ${ }^{96}$. Similar results were found in an intramuscular mouse infection model ${ }^{104}$. One group investigating host protection factors in a murine pneumonia model observed that $\beta 2$-microglobulin deficient mice, which are iron overloaded due to poor hepcidin production, were strikingly susceptible to K.pneumoniae infection; however, the authors did not investigate iron overload as the potential mechanism of susceptibility ${ }^{105}$. It is interesting to note that not all K.pneumoniae strains display enhanced virulence in the context of iron overload; for example, one group reported that some, but not all, strains of K.pneumoniae can be made more virulent by adding iron to skin lesions in Guinea pigs ${ }^{106}$. Our collaborators at the University of California, Los Angeles have also observed that iron supplementation can enhance the growth of some clinical $K$. pneumoniae strains in human plasma, but not others. Interestingly, the number of siderophores encoded in the genome does not always coincide with the ability of iron to enhance $K$. pneumoniae growth in human plasma (personal correspondence).

\section{Conclusions}

A fast, robust, and inflammatory innate immune response is essential for host protection against $K$. pneumoniae, but the breadth of host factors that are important are still undefined. While iron uptake is essential to the pathogenesis of Klebsiella 
pneumonia and subsequent bacteremia, it is unknown if the host can limit microbial access to iron, besides the known role of lipocalin-2 in the sequestration of enterobactin. As some siderophores produced by Enterobactericeae have the highest affinity for iron of any known compound, it is unclear if targeting host iron availability is an effective therapy.

\subsection{The role of iron in invasive pulmonary aspergillosis}

\subsubsection{Pathogenesis of invasive pulmonary aspergillosis}

Invasive pulmonary aspergillosis is a devastating fungal pneumonia caused by the Aspergillus species, a hardy and ubiquitous mold, which afflicts immunocompromised patients. It is defined by penetration of fungal hyphae into the parenchyma, and frequently, the vasculature of the lung. Most $(90 \%)$ invasive aspergillosis cases are caused by Aspergillus fumigatus, although several other species

including $A$. nidulans and $A$. niger are known to cause disease ${ }^{107}$. Aspergillus spores, called conidia, are easily dispersed in the air due to their small size $(2-3 \mu \mathrm{m})$, and hydrophobic proteinaceous layer. On average, humans inhale hundreds of conidia a day, with a small portion reaching the terminal alveoli where they cannot be cleared by cilia. In healthy individuals, conidia are readily killed by alveolar macrophages, neutrophils, and monocyte-derived phagocytes, normally without activating $\mathrm{T}$ and $\mathrm{B}$ cell mediated immunity ${ }^{108}$.

In contrast, individuals with defects in neutrophil number, function, or reactive oxygen species (ROS) generation are unable to clear conidia before they germinate into hyphae and invade the lung interstitium, causing extensive damage (Figure 1.4). 
Aspergillus species are not professional human pathogens and have not adapted evolutionarily to grow in the host environment; they lack many classic virulence factors, and their role as opportunistic human pathogens is thought to arise from their status as hardy, ubiquitous, and thermotolerant molds. Invasive pulmonary aspergillosis is a prominent cause of morbidity and mortality chronic granulomatous disease patients, hematopoietic stem cell transplants recipients, cancer patients on corticosteroids, and patients undergoing immunosuppressive treatments for solid organ transplants ${ }^{109}$. The antifungal voriconazole has greatly improved disease prognosis; however, mortality remains very high at $30 \%-50 \%$, with prognosis depending mostly on the immune status of the patient ${ }^{110}$. As immunosuppression therapies that render individuals susceptible to infection are increasingly common, invasive aspergillosis disease burden is likely to rise. It is therefore warranted to explore new therapeutic options, with an emphasis on host factors that mediate successful defense against infection. 


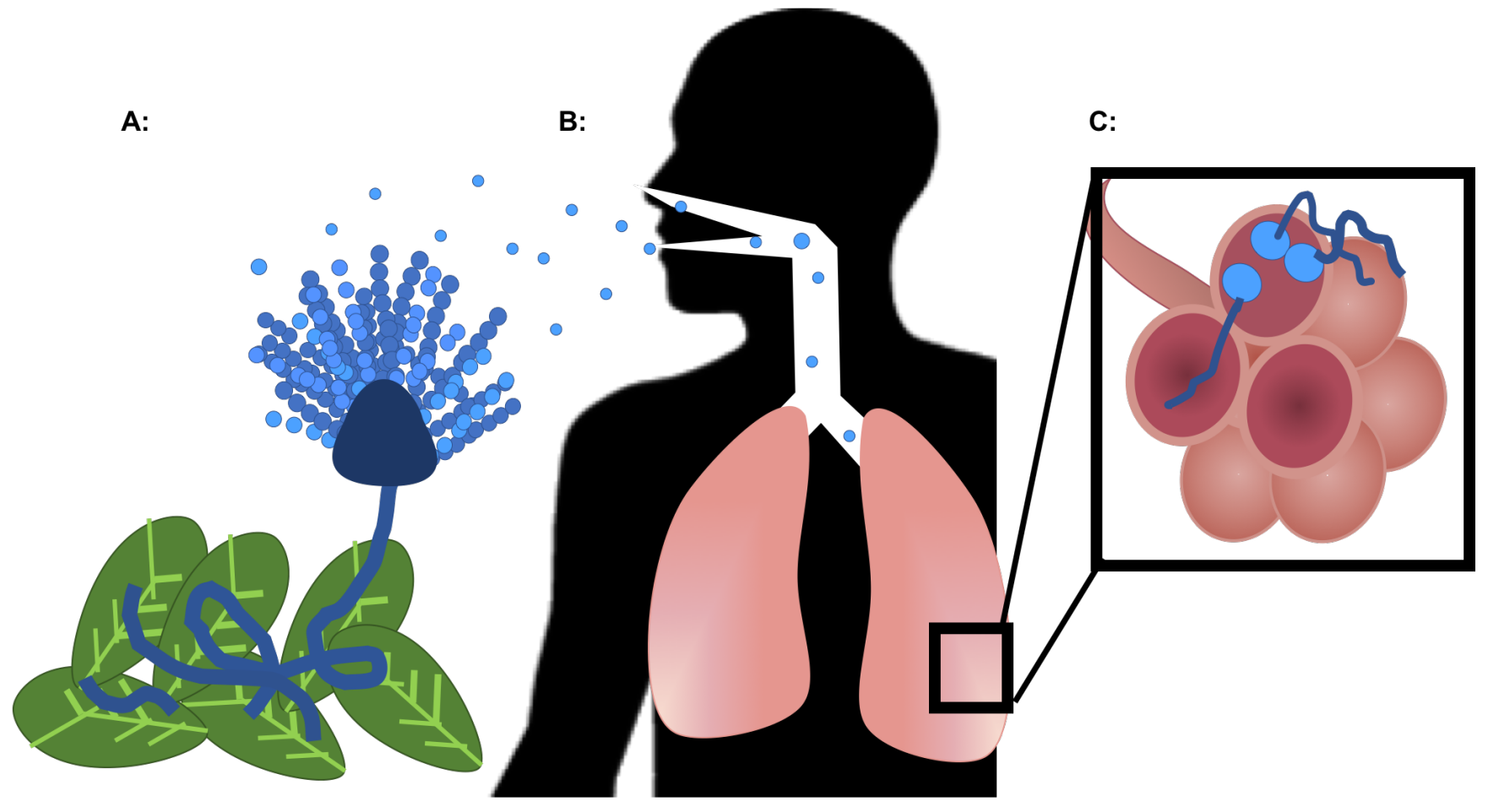


Figure 1.4: Growth cycle of pathogenic Aspergillus species. (A) Aspergillus species grow in organic debris found in the environment. The spores, known as conidia, are easily dispersed in the air. (B) Humans continually inhale conidia from the environment. Due to their small size, some conidia reach the terminal alveoli. In healthy humans, the conidia are easily cleared from the lower airways. (C) In profoundly immunocompromised individuals, conidia can germinate into hyphae and penetrate the lung parenchyma. 


\subsubsection{Host response against invasive pulmonary aspergillosis}

Similar to humans, mice with functional neutrophils have an extremely high resistance to Aspergillus conidia and will not develop invasive pulmonary aspergillosis at any inoculum size; therefore, all studies examining host defense against invasive pulmonary aspergillosis take place in the context of profound immunosuppression. In mice, this is normally achieved by corticosteroid treatment or antibody-mediated neutrophil depletion. Alveolar macrophages are the first to encounter and clear conidia inhaled into the lower airways. Resting conidia are covered in a hydrophobic, proteinaceous layer and are immunologically inert until they mature and expose PAMPs such as $\beta$-glucan ${ }^{111}$. In the absence of neutrophils, alveolar macrophages can be overwhelmed as conidia germinate into hyphae ${ }^{112}$. Defense is mediated by a large influx of inflammatory monocytes and an intrapulmonary accumulation of TNF-producing dendritic cells, some of which migrate to and present antigen in the draining lymph node to initiate adaptive immunity ${ }^{113} . T_{h} 1$ immune responses are protective whereas $T_{h} 2$ responses are detrimental ${ }^{114}$. There are conflicting reports as to whether a $T_{h} 17$ response is helpful or harmful, but the role of $T_{h} 17$ may depend on the underlying microbiome of the experimental animals $\mathbf{s}^{115,116}$

\subsubsection{The role of iron in invasive pulmonary aspergillosis}

Aspergillus species need iron for many essential processes, including hyphal growth, conidiation, and resistance to oxidative stress ${ }^{117}$. Several heme-containing proteins have been studied for their importance during infection. The cytochrome P450 sterol $14 \alpha$-demethylase enzyme (CYP51) is an enzyme required for ergosterol synthesis and is an essential component of the fungal cell wall. The CYP51 heme- 
containing pocket is the target of triazoles, which act to inhibit ergosterol synthesis by interfering with CYP51 function, and is mutated in triazole-resistant strains ${ }^{118}$. The hyphal catalases Cat1 and Cat2 have been shown to promote virulence in a rat infection model $^{119}$

Iron uptake is considered an important factor in invasive pulmonary aspergillosis. Aspergillus species produce four siderophores, two intracellular and two extracellular, which play essential and complementary roles in virulence ${ }^{120,121}$. The common precursor for all four siderophores is ornithine, which is converted to $\mathrm{N}^{5}$ hydroxyornithine by SidA (Figure 1.5) ${ }^{122,123}$. $\Delta$ SidA mutants are unable to synthesize siderophores, and are avirulent in animal infection models ${ }^{120}$. The extracellular siderophores fusarinine $\mathrm{C}$ (FusC) and triacetylfusarinine $\mathrm{C}$ (TAFC) are secreted by $A$. fumigatus to scavenge iron from the environment, which are transported back into the cell, and then hydrolyzed to release iron intracellular for metabolic use or storage . $^{124-126}$. Mutants deficient in extracellular siderophore production display completely abrogated virulence in murine infection models ${ }^{124}$. The intracellular siderophores ferricrocin (FC) and hydroxyferricrocin (HFC) mediate iron storage and distribution in A. fumigatus hyphae, and are also important for virulence in murine infection models ${ }^{124,127}$. Although A. fumigatus can acquire iron from the environment via low affinity ferrous iron uptake and reductive iron assimilation via the transporter FreA, these pathways are dispensable in the context of infection ${ }^{120}$. Siderophore production and utilization is mediated by HapX, a transcription factor which is essential for Aspergillus survival under iron starvation conditions and is required for virulence ${ }^{128,129}$. 


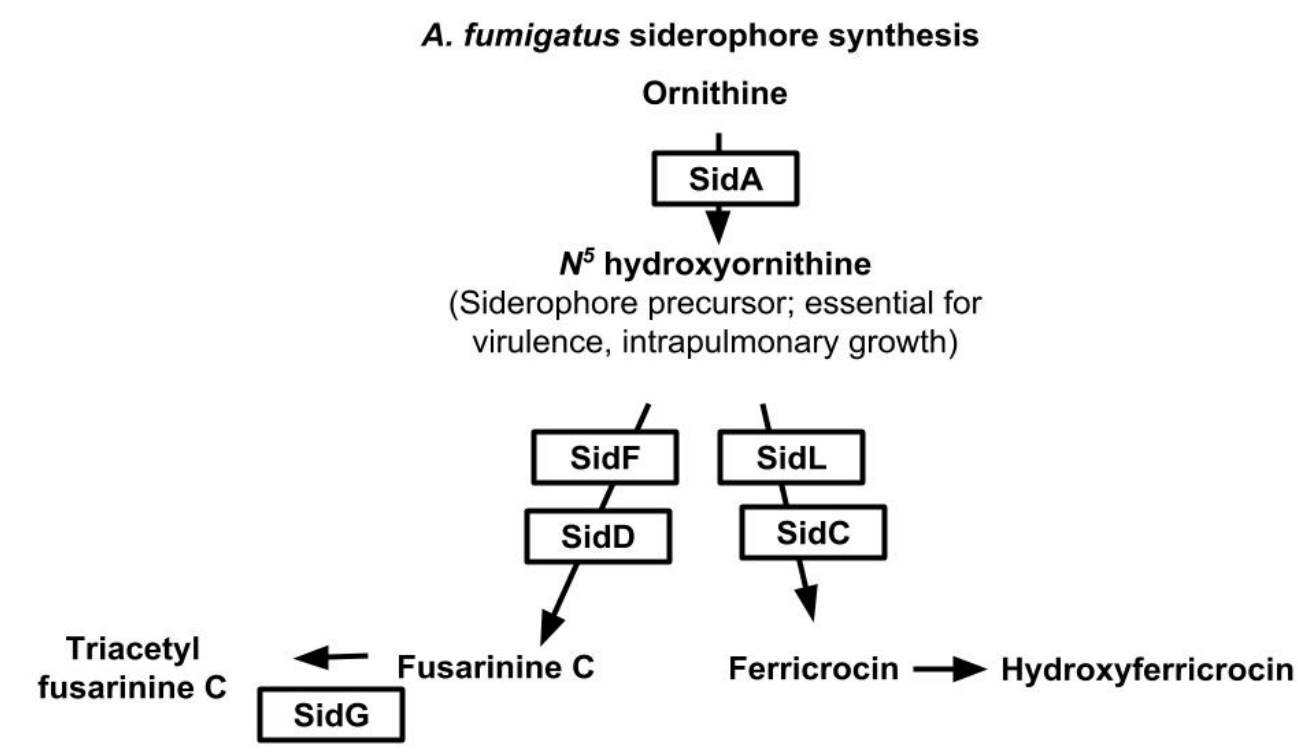

Extracellular siderophores: Important for full virulence, iron scavenging
Intracellular siderophores: Important for full virulence, growth, and stress 
Figure 1.5. Siderophore synthesis in Aspergillus species. Aspergillus synthesize two intracellular siderophores and two extracellular siderophores produced from the common precuorsor, $\mathrm{N}^{5}$ hydroxyornithine, which have crucial and complementary roles in virulence. Boxes represent enzymes that mediate the known steps of siderophore synthesis. 


\subsubsection{Evidence that host iron levels are important in invasive aspergillosis pathogenesis}

While profound immunosuppression is the most important risk factor for invasive pulmonary aspergillosis, there is correlative human data suggesting that host iron overload is an independent risk factor for infection. Liver transplant patients with hepatic iron overload develop invasive aspergillosis at higher frequencies than transplant patients without hepatic iron overload ${ }^{130}$. Similarly, stem cell transplant recipients with high liver or bone marrow iron stores had a higher risk for developing invasive aspergillosis compared to stem cell transplant recipients with normal iron stores ${ }^{131,132}$. While these studies suggest a connection between iron overload and susceptibility, it is difficult to fully separate the other negative effects that iron overload has on the overall status of human patients.

In addition to human correlative data, the importance of iron acquisition in invasive aspergillosis has been tested experimentally. One group found that lactoferrin, a host-derived iron chelator, is sufficient to inhibit $A$. fumigatus growth in vitro ${ }^{133}$. Recently, another group showed that $A$. fumigatus hyphae cultured in vitro formed hyphal branches to evade interactions with neutrophils in an iron-dependent manner ${ }^{134}$. Together, these studies suggest that neutrophil-mediated iron sequestration is important in invasive aspergillosis. This idea is further supported by the observation that the iron chelator deferasirox, combined with amphotericin B treatment, protects mice from invasive pulmonary aspergillosis, suggesting that restricting iron availability is a viable method for controlling infection ${ }^{135}$. 


\subsubsection{Heme uptake during invasive pulmonary aspergillosis}

It is widely stated throughout the literature that Aspergillus species have no mechanisms to uptake heme or hemoglobin. The evidence used to support this claim is the observation that heme supplementation does not seem to enhance $A$. fumigatus growth, and that SidA and FreA mutants are unable to grow on media containing heme as the sole iron source ${ }^{120}$. Although these observations indicate that heme is neither necessary nor sufficient to promote $A$. fumigatus growth, they do not eliminate the possibility of heme uptake. There are several lines of evidence that suggest Aspergillus species can utilize exogenous heme. Aspergillus species are used for industrial-scale production of heterologous proteins. It has been observed that Apergillus species genetically engineered to produce heme-containing proteins display enhanced production when cultures are supplemented with heme ${ }^{136}$; interestingly, certain proteins cannot be produced at all without heme supplementation ${ }^{137,138}$. A separate group studying heme metabolism in Aspergillus species observed that a mutant unable to synthesize heme could be rescued if grown in a rich media containing heme ${ }^{139}$. Together, these observations suggest that exogenous heme is accessible to Aspergillus species.

\subsection{Rationale for interrogating the role of iron sequestration and uptake during pneumonia}

As discussed in the previous sections, the ability of bacteria, parasites, and fungi to scavenge iron from their vertebrate hosts is a crucial, and sometimes defining, feature that separates pathogenic microbes from innocuous ones. The ability of 
mammalian hosts to effectively sequester iron similarly dictates susceptibility or resistance to infectious disease. Targeting iron acquisition and sequestration pathways is therefore a rational strategy to develop new treatment options against infections that do not currently have satisfactory treatments. The purpose of this thesis is to investigate the importance of iron metabolism in the two models of pneumonia discussed, Gram-negative pneumonia and invasive pulmonary aspergillosis, with the ultimate goal of identifying new therapeutic options.

Current treatments against Gram-negative pneumonia are failing due to the rise of antibiotic resistance. Disseminated disease in susceptible individuals caused by antibiotic-sensitive Gram-negative bacteria is often refractory to early an appropriate therapy for reasons that are not well-understood. While bacteria can mutate the cellular targets of antibiotics, the need for iron cannot be evaded. Hepcidin, the master iron regulatory hormone, is induced and inhibited by a complex array of conditions that are commonly seen in patient groups who are susceptible to nosocomial Gram-negative infections. As there are currently numerous products in development to either inhibit or replace hepcidin in these patient groups for the treatment of iron storage disorders, the focus of chapter II is the role of hepcidin in Gram-negative pneumonia with an emphasis on how altering global iron regulation mediates defense against disease.

Invasive pulmonary aspergillosis has a poor prognosis despite modern antifugnal treatment. While the role of iron uptake has been intensely studied, the role of heme uptake, the largest iron pool in mammalian hosts, has so far been neglected and prematurely dismissed. There is evidence that Aspergillus species can utilize exogenous heme under certain circumstances, but it is unknown if heme uptake 
promotes disease during human infection. The overall purpose of chapter III is to investigate heme uptake as a novel virulence mechanism, and therefore a novel therapeutic pathway, in invasive pulmonary aspergillosis. 


\section{Chapter II: The role of hepcidin in Gram-negative pneumonia}

Michels, K. R. et al. Hepcidin-mediated iron sequestration protects against bacterial dissemination during pneumonia. JCI Insight 2, (2017).

\subsection{Introduction}

Infections caused by aerobic Gram-negative bacteria are prevalent and dangerous. As a group, these organisms are the most common etiology of nosocomial infections, including most cases of hospital-acquired pneumonia, ventilator-associated pneumonia and healthcare-associated pneumonia, and are important causes of morbidity and death in hospitalized patients. Dissemination of these organisms from the primary site of infection to the bloodstream is especially associated with mortality ${ }^{140,141}$. The progressive rise in antibiotic resistance among Gram-negative bacteria, particularly in the healthcare setting, has critically diminished the available treatment options for these infections. Better mechanistic understanding of the host-pathogen interplay during Gram-negative infections has the potential to lead to novel therapeutic strategies to augment the diminishing armamentarium of traditional antibiotics.

Iron is an essential component of many proteins and a required nutrient for nearly all organisms. In biological systems, free iron ions are present at extremely low concentrations and the competition for iron between pathogens and the host is important to the outcome of many infections ${ }^{2}$. While the iron acquisition mechanisms of aerobic Gram-negative are known to be essential to their virulence ${ }^{142}$, the host mechanisms of iron restriction in these infections are incompletely defined. 
Under homeostatic conditions, plasma iron is rapidly turned over with a half-life of $<1 \mathrm{~h}$, being continuously utilized in erythropoiesis and replenished by splenic macrophages that scavenge iron from senescent erythrocytes ${ }^{4}$. The hormone hepcidin is the key regulator of plasma iron concentration that acts by mediating the degradation of the only known cellular iron exporter, ferroportin, thus trapping iron inside cells and reducing extracellular iron ${ }^{10}$. During homeostasis, hepcidin is induced by elevated extracellular and intracellular iron levels via SMAD4 signaling, and is inhibited by anemia and hypoxia. During inflammation, hepcidin in induced as an acute-phase reactant by IL-6 and Activin B via STAT3 and SMAD4, respectively ${ }^{9,143}$. In the context of inflammation, hepcidin mediates acute hypoferremia that is posited to be a defense mechanism against infectious disease; continual hepcidin production in this setting can infamously cause anemia of chronic inflammation. The role of hepcidin in disorders of iron metabolism is well-established, but its contribution to antimicrobial host defenses is an emerging field: For example, hepcidin appears to protect the host against malaria but to promote the growth of pathogens that reside within the intracellular niche of macrophages ${ }^{144}$. Hepcidin-mediated hypoferremia is essential for protection of the host against Vibrio vulnificus in a murine model ${ }^{55}$, consistent with the clinical observation that individuals with iron-overload conditions are uniquely susceptible to this organism ${ }^{11}$. In contrast, the role of hepcidin as a general defense against common bacterial infections, such as those caused by Enterbacteriaceae, remains unknown.

We reasoned that the bloodstream dissemination of microorganisms from the primary site of infection is a critical complication in Gram-negative bacterial infections: In the context of pneumonia, as well as other focal infections caused by aerobic Gram- 
negative bacilli, bacteremia is strongly associated with sepsis and death ${ }^{145,146}$. We therefore tested the hypothesis that hepcidin-mediated depletion of extracellular iron during Gram-negative pneumonia protects the host by limiting dissemination of bacteria to the bloodstream. 


\subsection{Results}

\subsubsection{Plasma iron is suppressed during bacterial pneumonia}

We used a well-described model of Klebsiella pneumonia caused by a virulent strain of bacteria, delivered directly into the trachea of sedated experimental mice ${ }^{92,147}$. We first characterized the effect of Klebsiella pneumonia on iron metabolism in wildtype mice. As expected, plasma iron concentration decreased quickly in wildtype mice following infection (Figure 2.1A), similar to prior reports with acute inflammatory stimuli ${ }^{9}$. To assess the availability of iron to bacteria in the lungs, we also measured hemeassociated and non-heme-associated iron levels in whole lung homogenates (which includes both intra- and extra- cellular iron) and in the extracellular space, in the bronchoalveolar lavage (BAL) supernatant (Figure 2.1B-C). We found that most lung iron was intra-cellular, and extracellular iron levels were low and did not change appreciably during infection.

\subsubsection{Increased iron availability worsens the outcome of infection}

To determine whether iron availability influences host defense during the course of infection, we tested the effect of transient increase in extracellular iron availability, achieved by intraperitoneal injection of ferric ammonium citrate, on the outcome of Klebsiella pneumonia. Mice treated with iron prior to intrapulmonary $K$. pneumoniae challenge developed transient elevation in plasma iron that resolved completely within twelve hours (Figure 2.1D) without influencing lung iron (Figure 2.S1), consistent with prior studies indicating that excess iron is rapidly cleared from the blood and redistributed to the bone marrow, spleen and liver ${ }^{4}$. This transient increase in iron 
availability during the first $24 \mathrm{~h}$ after infection was associated with a markedly increased mortality from pneumonia associated with increased bacterial burden in the lung and the blood (Figure 2.1E-F). Since wildtype mice developed hypoferremia upon infection with K. pneumoniae, and excess iron was associated with poor infection outcome, we hypothesized that endogenous iron regulation was important for controlling infection. 
A: Plasma

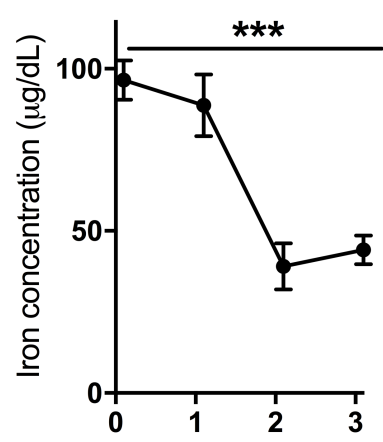

D: Iron treatment

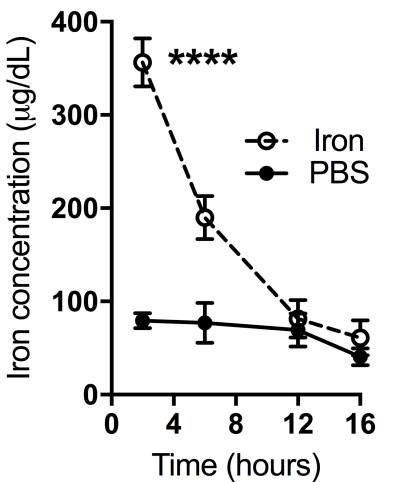

B: Lung
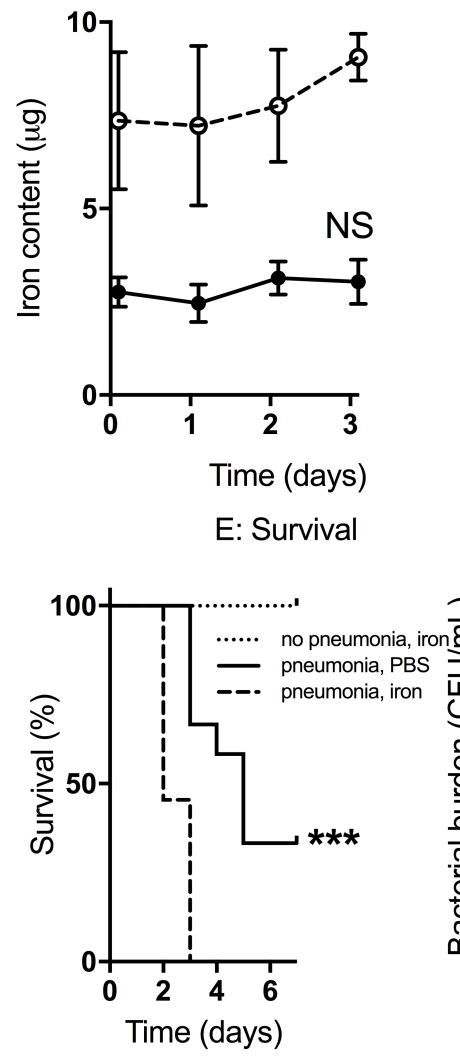

C: BAL

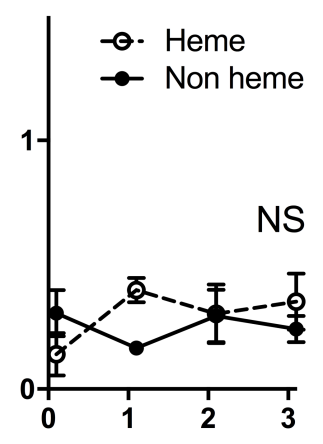

F: Day 2 bacterial burden

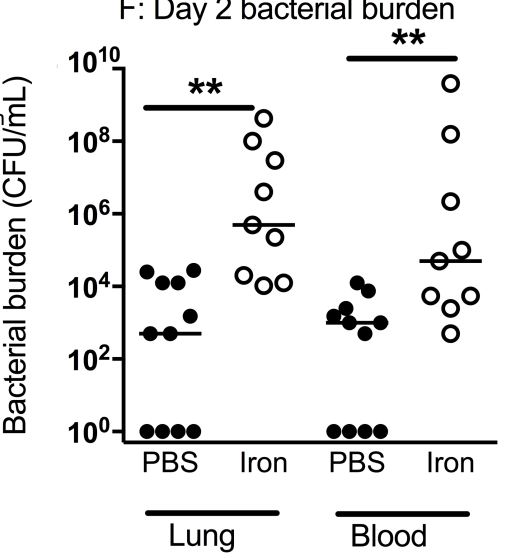


Figure 2.1. Iron availability during Klebsiella pneumonia. Time 0 represents uninfected animals. (A)): Iron concentration in the plasma. (B-C) Calculated total iron levels in whole lung homogenate from a single mouse, and bronchoalveolar lavage fluid (BAL) recovered in a volume of $1 \mathrm{~mL}$ from a single mouse. Data shown as mean \pm standard error (SE); $n=4-6$ animals per time point, representative of 2 independent experiments; ${ }^{* * * *}, p<0.0001$; NS $=$ Not significant $(1$-way ANOVA, multiple comparison for linear trend). (D): Plasma iron following intraperitoneal injection with PBS or ferric ammonium citrate (iron) 30 minutes prior to intrapulmonary K. pneumoniae challenge. $n$ $=6-8$ per group per time point, combined result of 2 experiments; ${ }^{* * *}, p<0.001$, Twoway ANOVA. (E): Mice were treated with either PBS or ferric ammonium citrate (iron) 30 minutes prior to intrapulmonary $K$. pneumoniae challenge and again 24 hours thereafter. $n=6-12$ per group, combined result of 2 experiments; ${ }^{* *}, p<0.01$ MantelCox test. $(F)$ : Bacterial burden on day two of infection in mice treated with ferric ammonium citrate or PBS prior to intratracheal challenge with K. pneumonia. Horizontal lines represent median and each circle represents one animal; animals with no detectable bacteria are reported to have a bacterial burden of 1 colony forming units on the logarithmic scale. $n=9-11$, combined result of 2 experiments; ${ }^{* *}, p<0.01$ MannWhitney test. 


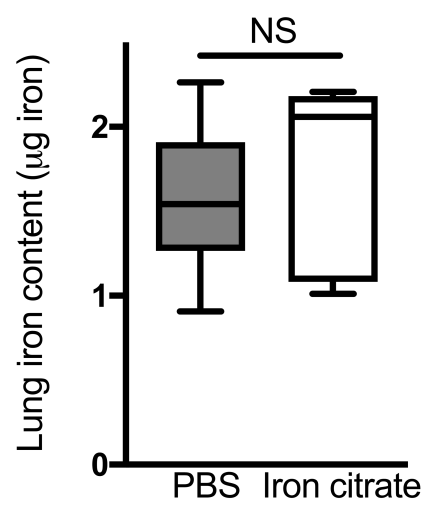


Figure 2.S1. Lung iron content in wildtype mice treated with PBS or iron citrate. Mice were treated with either PBS or ferric ammonium citrate (iron) 30 minutes prior to intrapulmonary K. pneumoniae challenge and again 24 hours thereafter; lungs were harvested on day 2 of infection. Lung iron content was determined using the ferrozine assay. Box and whisker plots show median (line within box), upper and lower quartiles (upper and lower box boundaries), and total range (bars); $n=6-5$ per group; NS, not significant (t-test). 


\subsubsection{Hepcidin regulates plasma iron during bacterial pneumonia by an IL-6- dependent mechanism}

To assess the role of hepcidin in mediating hypoferremia during pneumonia, we began by characterizing hepcidin expression during the infection. Infection resulted in a marked increase in hepcidin protein levels in plasma, in parallel with the development of hypoferremia (Figure 2.2A). Hepcidin protein concentration also increased in the lung, but at relatively low concentrations $(\sim 15 \mathrm{ng}$ in the entire lung, as compared to $\sim 1500$ $\mathrm{ng} / \mathrm{mL}$ of plasma on day 3; Figure 2.2B). The liver is the dominant source of hepcidin at baseline $e^{7,12}$, but hepcidin can be induced in leukocytes and airway epithelial cells in response to inflammatory stimuli under in vitro conditions ${ }^{66,39,26}$. We thus measured hepcidin transcription in the liver, blood, and lungs during infection and found a robust induction of hepcidin mRNA in the liver (Figure 2.2C) but not in peripheral blood leukocytes or lungs (Figure 2.2D-E), suggesting that liver is the main source of hepcidin during the infection and elevations in lung hepcidin protein likely represent extravasation from the blood.

The cytokine IL-6 is a key inducer of acute phase proteins in the liver, and an important regulator of hepcidin expression in vivo ${ }^{9}$. IL-6 is induced in the lungs in this model of pneumonia ${ }^{148}$. We found that immuno-neutralization of IL-6 resulted in higher blood and lung bacterial burden two days following infection (Figure 3.3A). In addition, IL-6 neutralization abrogated the increase in plasma hepcidin during the infection and eliminated the associated hypoferremia (Figure 2.3B-C), indicating that hepcidin induction during pneumonia is dependent on IL-6. 


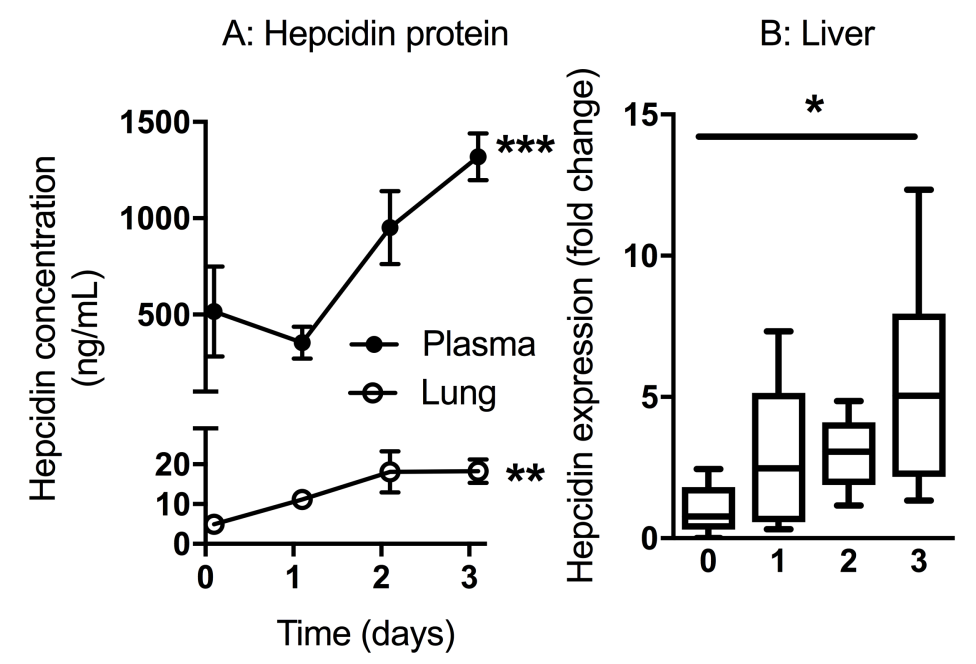

C: Buffy coat $\quad$ D: Lung

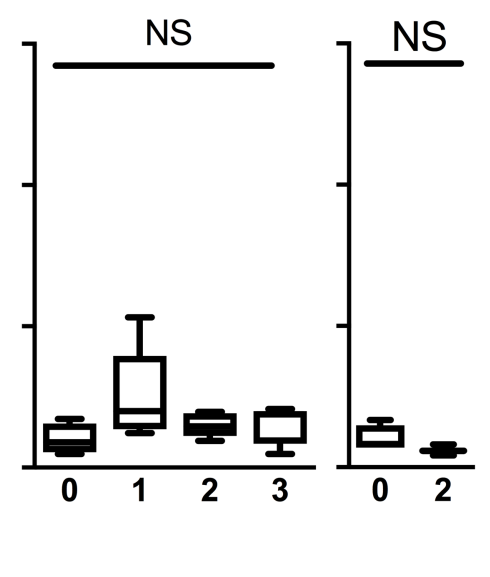


Figure 2.2. Hepcidin induction during Klebsiella pneumonia. Time 0 represents naïve animals. (A): Hepcidin protein levels in the plasma and whole lung homogenate following infection. Data represent mean $\underline{\underline{S}}$ SEM. (B-D): Hepcidin mRNA in the liver, buffy coat, and lung normalized to GAPDH expression, and then to day 0. Box and whisker plots show median (line within box), upper and lower quartiles (upper and lower box boundaries), and total range (bars); $n=4-6$ animals per time point in each panel; *, ${ }^{* *}$ and ${ }^{* * *}$ denote $p$ values of $<0.05,<0.01$ and $<0.001$, respectively; NS, not significant (1-way ANOVA, multiple comparison for linear trend for panels A-C, t-test for panel D). 
A: Day 2 bacterial burden

B:

C:
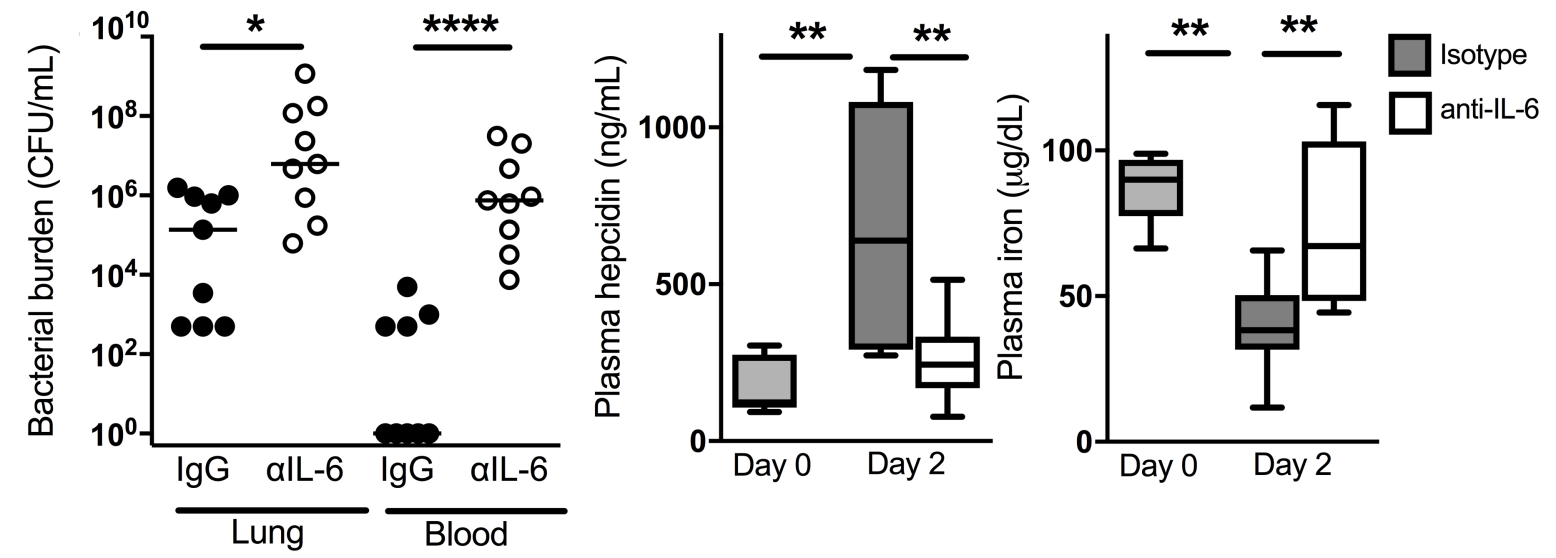
Figure 2.3: The role of IL-6 in hepcidin induction during Klebsiella pneumonia. (A): Animals were treated daily with an isotype control or IL-6 neutralizing monoclonal antibody and assessed for bacterial burden on day 2 of infection. Horizontal lines represent median and each circle represents one animal; animals with no detectable bacteria are reported to have a bacterial burden of 1 colony forming units on the logarithmic scale; * and ${ }^{* *}$ denote $p$ values of $<0.05$ and $<0.01$ by Mann-Whitney test, respectively. (B-C): Plasma hepcidin protein and iron levels in uninfected and infected animals treated with isotype or IL-6 neutralizing antibody. Box and whisker plots show median (line within box), upper and lower quartiles (upper and lower box boundaries), and total range (bars) combined from 2 experiments; $n=8-9$ animals per group; ${ }^{* *}, p$ $<0.01$, one way ANOVA Tukey post-test. 


\subsubsection{Hepcidin mediates hypoferremia during bacterial pneumonia and is required for survival}

Reduced availability of plasma iron in the context of acute inflammation can occur as a result of both hepcidin-dependent and -independent mechanisms ${ }^{149-151}$. We therefore tested the contribution of hepcidin in regulation of plasma iron levels in the context of bacterial pneumonia. In contrast to reduction in plasma iron during infection in wildtype mice, plasma iron in hepcidin-deficient mice became more elevated during the infection (Figure 2.4A), indicating that hepcidin is essential for controlling plasma iron levels following infection. Consistent with reports of tissue iron overload in hepcidindeficient mice ${ }^{12,152}$, we found elevated lung iron levels in hepcidin-deficient as compared to wildtype mice, but these values did not change over the infection period (Figure 2.4B).

We next assessed the contribution of hepcidin-mediated iron sequestration to host defense against $K$. pneumoniae and found that hepcidin-deficient animals were markedly more susceptible to bacterial pneumonia, demonstrating $100 \%$ mortality compared to $50 \%$ mortality in wildtype controls (Figure $2.4 \mathrm{C}$ ), comparable to mice treated with exogenous iron during infection (Figure 2.1D-E). Hepcidin-deficient mice exhibited $23 \%$ greater incidence of bacteremia and $>100$-fold higher median blood bacterial concentration, as well as $\sim 10$-fold higher bacterial burden in the lungs, and liver as compared to wildtype mice (Figure 2.4D).

Since the hepatocytes in hepcidin-deficient animals are iron-loaded, we reasoned that the increase of iron in infected hepcidin-/- mice could originate from greater release of iron from damaged hepatocytes. We therefore quantified alanine aminotransferase 
and aspartate aminotransferase in the blood of infected mice to assess liver damage, and found neither enzyme to be elevated in hepcidin-/- as compared to wildtype mice in the context of infection (Figure 2.S2). These data argue against disproportionate hepatic injury as a contributor to worse outcomes in hepcidin-deficient hosts.

In order to assess whether the beneficial effects of hepcidin are dependent on iron, we used established protocols to induce severe iron-deficiency in hepcidindeficient mice ${ }^{153}$. We found that iron-depleted hepcidin-/- animals did not develop hyperferremia during infection, had lower body iron stores than wildtype mice, and were no more susceptible to infection compared to wildtype mice (Figure 2.S3A-C). Taken together, these data suggest that the hepcidin-mediated control of bacterial growth during infection is iron-dependent. 

A: Plasma
B: Lung
C: Survival
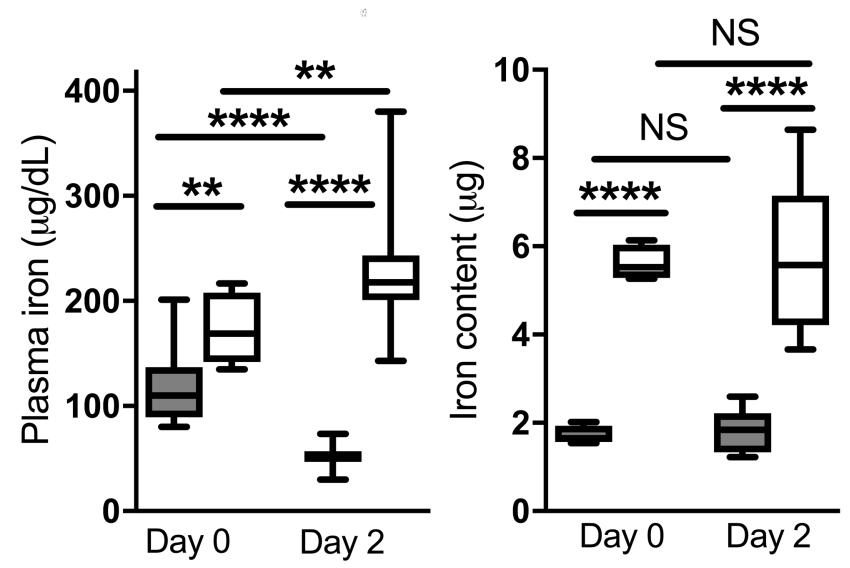

$\square$ WT $\square$ Hepcidin -/-
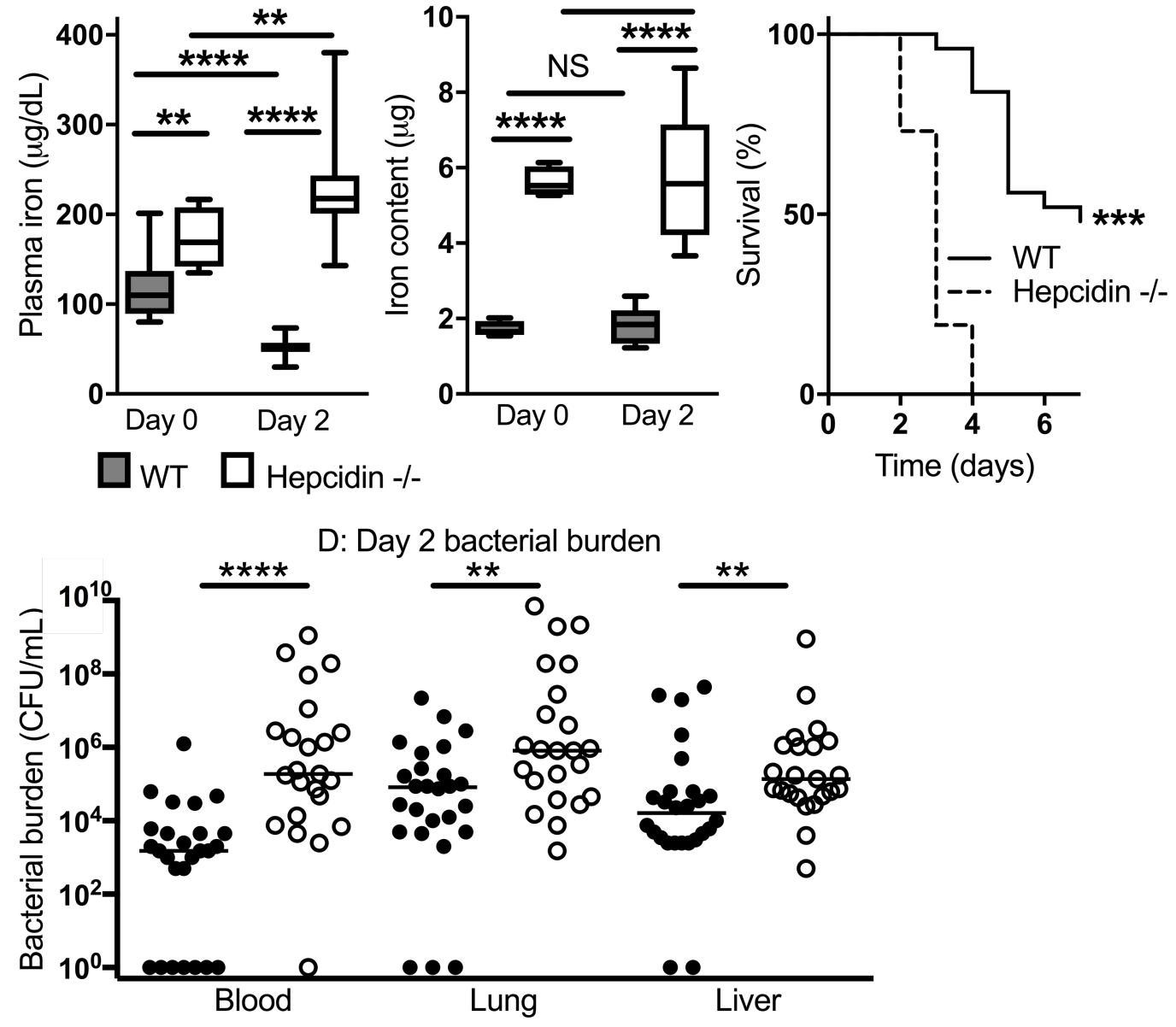
Figure 2.4: The role of hepcidin in Klebsiella pneumonia and dissemination. (A-B): Comparison of plasma or lung non-heme iron levels in WT and hepcidin-/- mice before and 2 days post infection. Box and whisker plots show median (line within box), upper and lower quartiles (upper and lower box boundaries), and total range (bars) of data from 2 experiments; $n=4-9$ per group; ${ }^{* *}$ and ${ }^{* * * *}$ denote $p$ values of $<0.01$ and $<0.0001$ by one-way ANOVA with Tukey post test. (C): Survival of WT and hepcidin-/mice during pneumonia. $N=26-30$ mice per group, combined from 3 independent experiments; ${ }^{* * *}, p<0.0001$ Mantel-Cox test. (D): Bacterial burden on day 2 of infection in WT and hepcidin-/- mice in indicated organs. Pooled data from 3 independent experiments; $n=23-26$. Horizontal lines represent median and each circle represents one animal; animals with no detectable bacteria are reported to have a bacterial burden of 1 colony forming units on the logarithmic scale; ${ }^{* *}$ and ${ }^{* * *}$ denote $p$ values of $<0.01$ and $<0.001$, respectively using the Mann-Whitney test. 

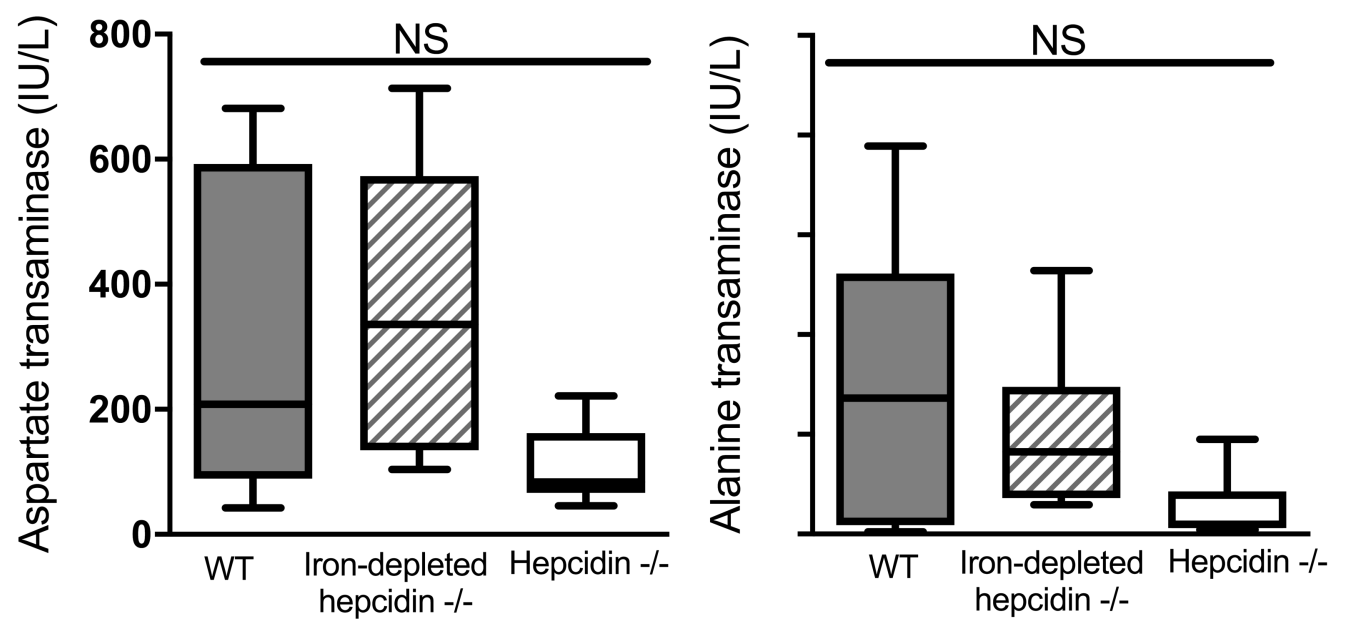
Figure 2.S2. Aspartate transaminase (AST) and alanine transaminase (ALT) in wildtype and hepcidin-/- mice. Wildtype (WT) mice were maintained on standard diet, and hepcidin-/- mice were maintained on wither a prolonged iron-deficient diet (Iron depleted hepcidin-/-) or standard diet prior to intratracheal inoculation with $K$. pneumoniae. Samples were harvested on day 2 of infection. Box and whisker plots show median (line within box), upper and lower quartiles (upper and lower box boundaries), and total range (bars); $n=6-9$ per group; NS=not significant, one-way ANOVA. 

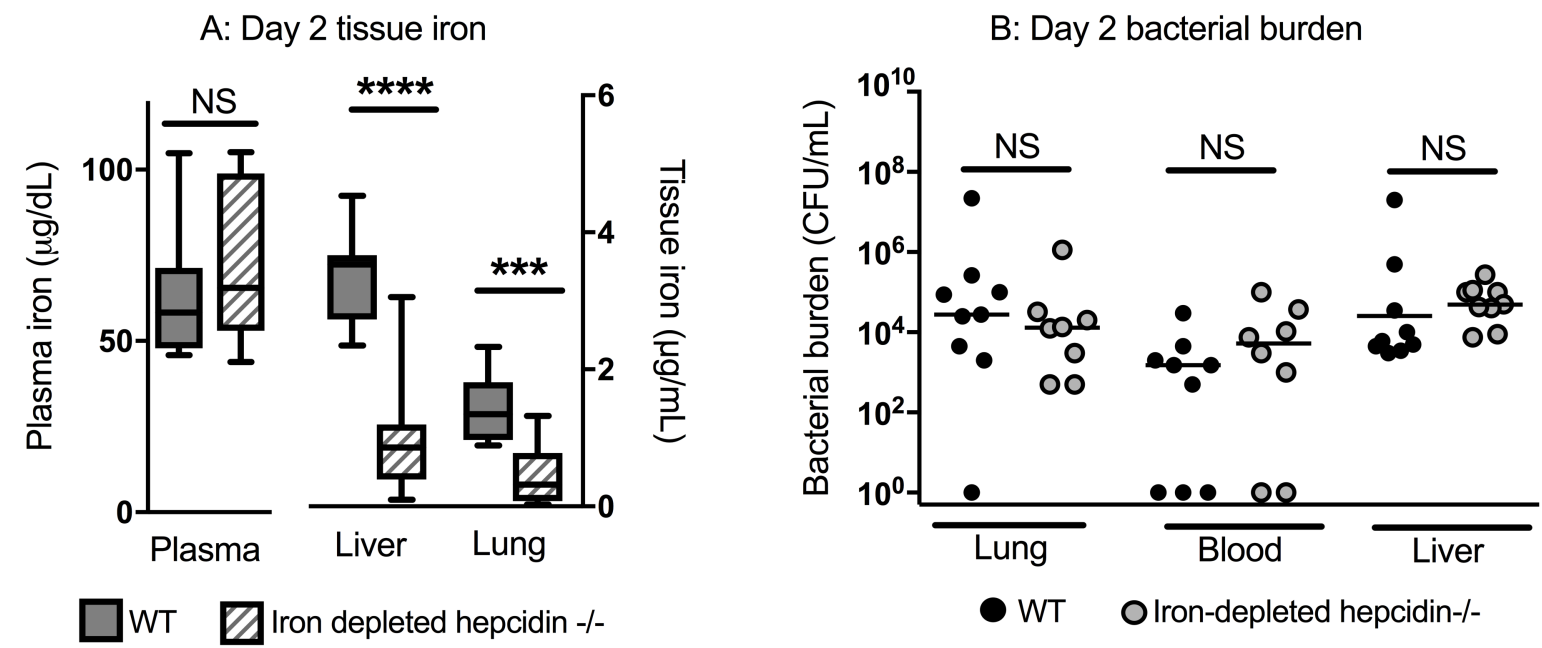
Figure 2.S3. Iron-depleted hepcidin-/- mice are not susceptible to infection compared to wildtype mice. Wildtype (WT) mice maintained on standard diet and hepcidin-/- mice maintained on a prolonged iron-deficient diet were infected intratracheally with K. pneumoniae and samples were harvested on day 2 of infection. (A) Liver, lung, and plasma iron content in wildtype and iron-depleted hepcidin-/- mice. Box and whisker plots show median (line within box), upper and lower quartiles (upper and lower box boundaries), and total range (bars); ${ }^{* * *}$ and ${ }^{* * * *}$ denote $p<0.001$ and $p$ $<0.0001$ respectively; NS, not significant (t-test). (B): Lung, blood, and liver bacterial burden in wildtype and iron-depleted hepcidin-/- mice. Horizontal lines represent median and each circle represents one animal; animals with no detectable bacteria are reported to have a bacterial burden of 1 colony forming units on the logarithmic scale; NS, not significant (Mann-Whitney test). $N=8-9$ per group in both panels. 


\subsubsection{Excess iron promotes the growth of $K$. pneumoniae in plasma}

To assess whether the increased susceptibility of hepcidin-deficient mice to infection was attributable to increased bacterial growth in the high-iron environment of the plasma, we assessed the effect of iron supplementation on the in vitro growth of $K$. pneumoniae in human plasma. Supplementing agar composed of $90 \%$ human plasma with ferric ammonium citrate at physiologically relevant concentrations incrementally raised the transferrin saturation of the sample, resulting in the appearance of nontransferrin-associated labile pool iron (Figure 2.5A). The appearance of labile pool iron at transferrin saturation greater than $70 \%$ is consistent with prior reports ${ }^{3,154} . \mathrm{K}$. pneumoniae growth was suppressed in plasma, but was permitted by iron supplementation, consistent with reports that transferrin inhibits bacterial growth ${ }^{96}$ (Figure 2.5B). Interestingly, we found that, in plasma preparations from 3 independent donors, addition of ferric iron resulted in $K$. pneumoniae out-growth coincided with the appearance of labile iron in the plasma, rather than with the total amount of iron citrate added. These data suggest that ferric ammonium citrate supports $K$. pneumoniae growth in plasma by providing iron, rather than acting as a carbon or nitrogen source.

We next sought to test the hypothesis that, during in vivo infection, elevated plasma iron is sufficient to enhance growth of blood-borne bacteria. To test this, we subjected wildtype mice with an intraperitoneal injection of PBS or ferric ammonium citrate prior to intravenous challenge with $K$. pneumoniae. After $24 \mathrm{~h}$, mice treated with ferric ammonium citrate had higher blood bacterial content than mice treated with PBS, indicating that elevated plasma iron directly mediates increased growth of $K$. pneumoniae in the blood stream (Figure 2.5C). Interestingly, mice treated with ferric 
ammonium citrate also had higher bacterial burdens in the lungs. We interpret this as evidence that, in mice with increased bacteremia, bacterial seeding of the lung from the bloodstream contributes to higher lung bacterial content. Similar to prior reports ${ }^{8}$, mice treated with iron also had marked elevation of plasma hepcidin as compared to mice treated with PBS in response to acute iron loading (Figure 2.5D). These results suggest that hepcidin induction alone is not sufficient to protect mice from fatal infection, and supporting the hypothesis that the beneficial effects of hepcidin are mediated via induction of hypoferremia, rather than an iron-independent effect. 

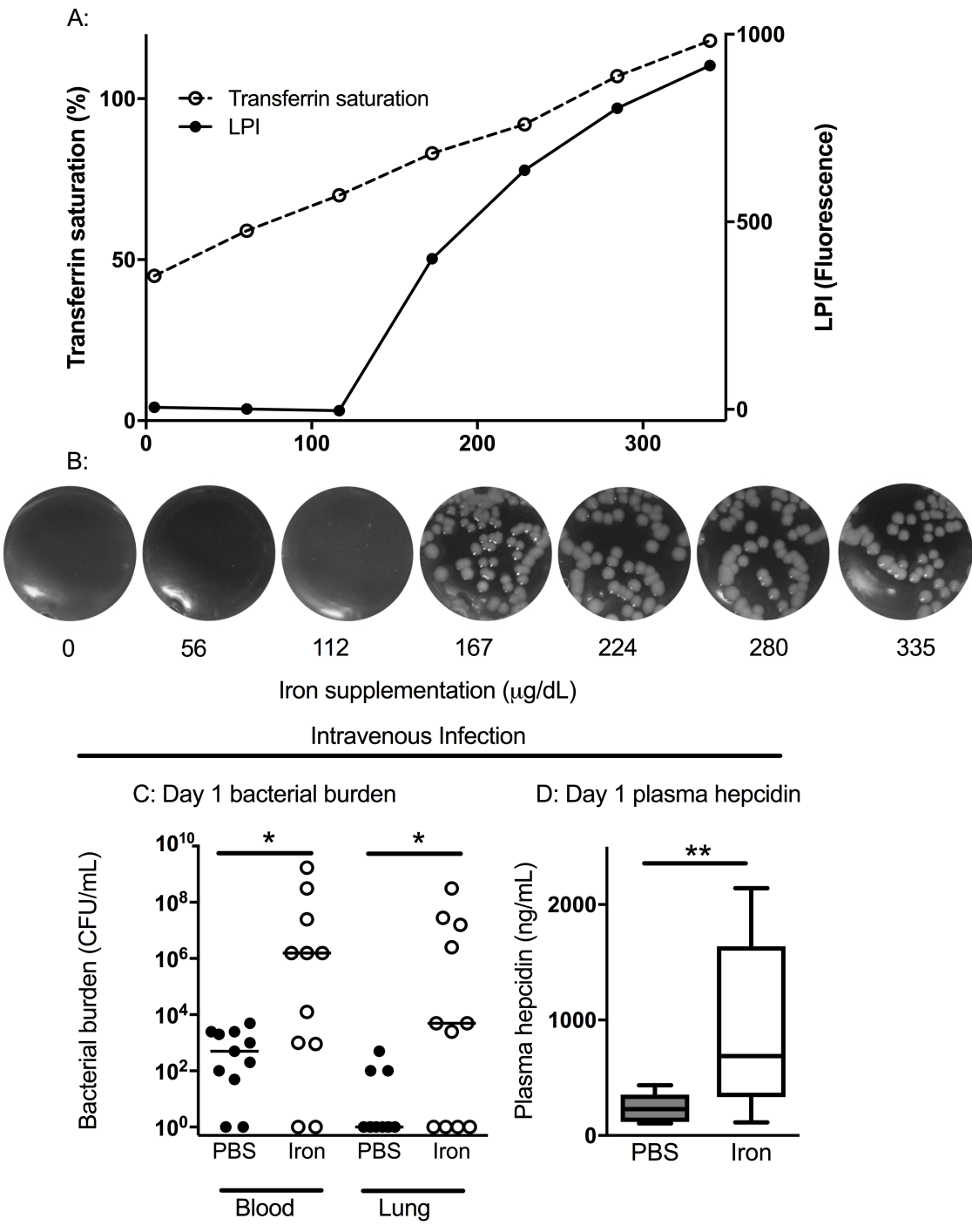
Figure 2.5. Effect of iron on Klebsiella growth in vitro and in vivo. (A): Human plasma was supplemented with ferric ammonium citrate and assayed for transferrin saturation and the presence of labile plasma iron (LPI). Transferrin saturation values of $>100 \%$ represent complete saturation of transferrin iron-binding sites in the presence of additional, unbound ferric iron ions. (B): Agar plates composed of $90 \%$ human plasma were supplemented with ferric ammonium citrate and inoculated with $K$. pneumoniae. Plates were monitored for occurrence of bacterial growth. Representative data from 3 independent plasma agar preparations. (C-D): Bacterial burden and plasma hepcidin protein in wildtype mice treated with either PBS or iron citrate 30 minutes prior to intravenous challenge with $K$. pneumoniae. Lungs and blood were harvested after 24 hours. Box and whisker plots show median (line within box), upper and lower quartiles (upper and lower box boundaries), and total range (bars). $N=11$ mice per group, combined form 2 experiments; *, $p<0.05$ Mann-Whitney test; ${ }^{* *}, p<0.01 t$-test. 


\subsubsection{Hepcidin-deficient mice have intact cellular immunity against Klebsiella}

Iron deficiency and iron overload have complex effects on immune responses ${ }^{155}$, and phagocytes from iron-overloaded hosts may have altered anti-bacterial properties that could contribute to impaired host defenses of hepcidin-deficient mice independent of the effects of acute hyperferremia on bacteria. In order to assess the functionality of the innate immune response in hepcidin-deficient mice, we analyzed leukocyte populations in the blood and BAL (Figure 2.6A-F). We found similar concentrations of circulating neutrophils, Ly6 $\mathrm{C}^{\mathrm{hi}}$ and $\mathrm{Ly}_{6} \mathrm{C}^{\mathrm{lo}}$ monocytes in wildtype and hepcidin-deficient mice at baseline and two days following infection. The number of BAL alveolar macrophages, neutrophils, and monocyte-derived macrophages were also similar between wildtype and hepcidin-deficient hosts, both at baseline and following infection, arguing against a defect in leukocyte quantity or recruitment in the absence of hepcidin. Since oxidative burst is an iron-dependent process, we compared the generation of reactive oxygen species in lung and blood leukocyte populations and found no difference between infected WT and hepcidin-deficient mice (Figure 2.S4). Similarly, neutrophils from wildtype and hepcidin-deficient mice exhibited comparable ex vivo bacterial killing (Figure 2.6G). Interestingly, we found that thioglycollate-elicited peritoneal macrophages from both wildtype and hepcidin-deficient mice were unable to kill $K$. pneumoniae even with overnight IFN- $\gamma$ treatment (data not shown). While these data do not formally exclude the possibility that hepcidin-deficiency impairs the immune system in some other way, they suggest intact cellular innate immune mechanisms against $K$. pneumoniae in hepcidin-/- mice. 

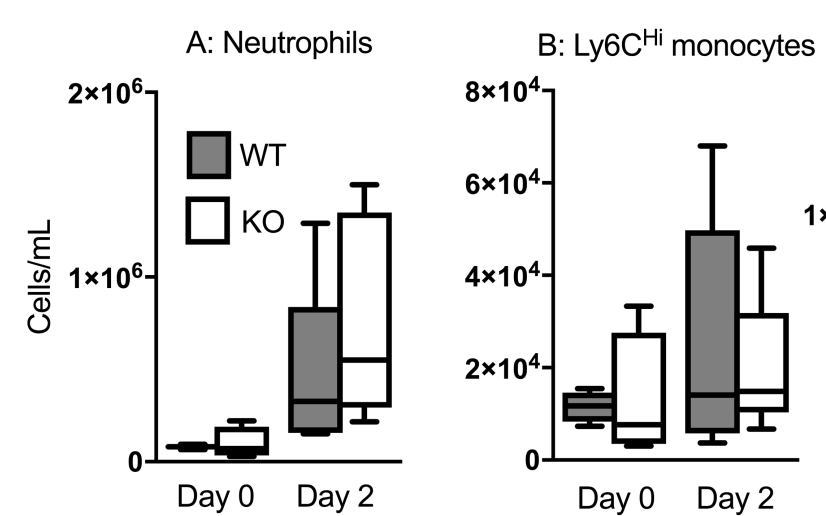

$$
\text { C: Ly6C } \mathrm{C}^{\mathrm{lo}} \text { monocytes }
$$
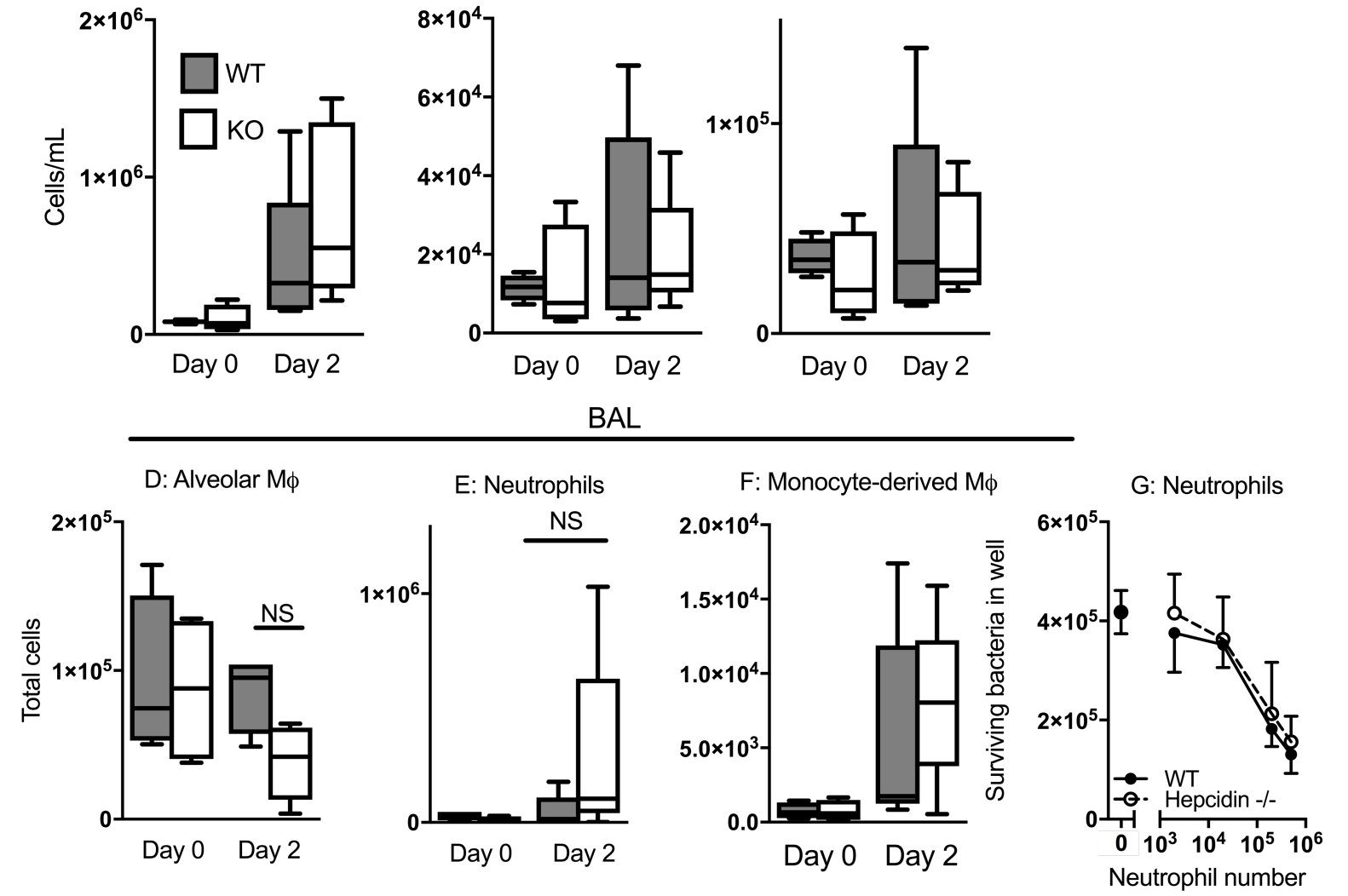
Figure 2.6. Leukocyte responses during Klebsiella pneumonia. (A-F): BAL and blood were harvested from WT and hepcidin-/- mice before and 2 days following infection. Box and whisker plots show median (line within box), upper and lower quartiles (upper and lower box boundaries), and total range (bars). $N=4-5$ per group, NS=not significant, one-way ANOVA Tukey post-test. No significant differences were found for any WT to hepcidin-/- comparison. (G): Freshly isolated neutrophils from WT and hepcidin-/- mice were co-incubated with $2 \times 10^{5}$ CFU of $K$. pneumoniae for 1 hour. Total surviving bacteria were enumerated from each well. $N=3$ independent experiments per group; data were analyzed using a 2 way ANOVA and no differences were found between groups. 


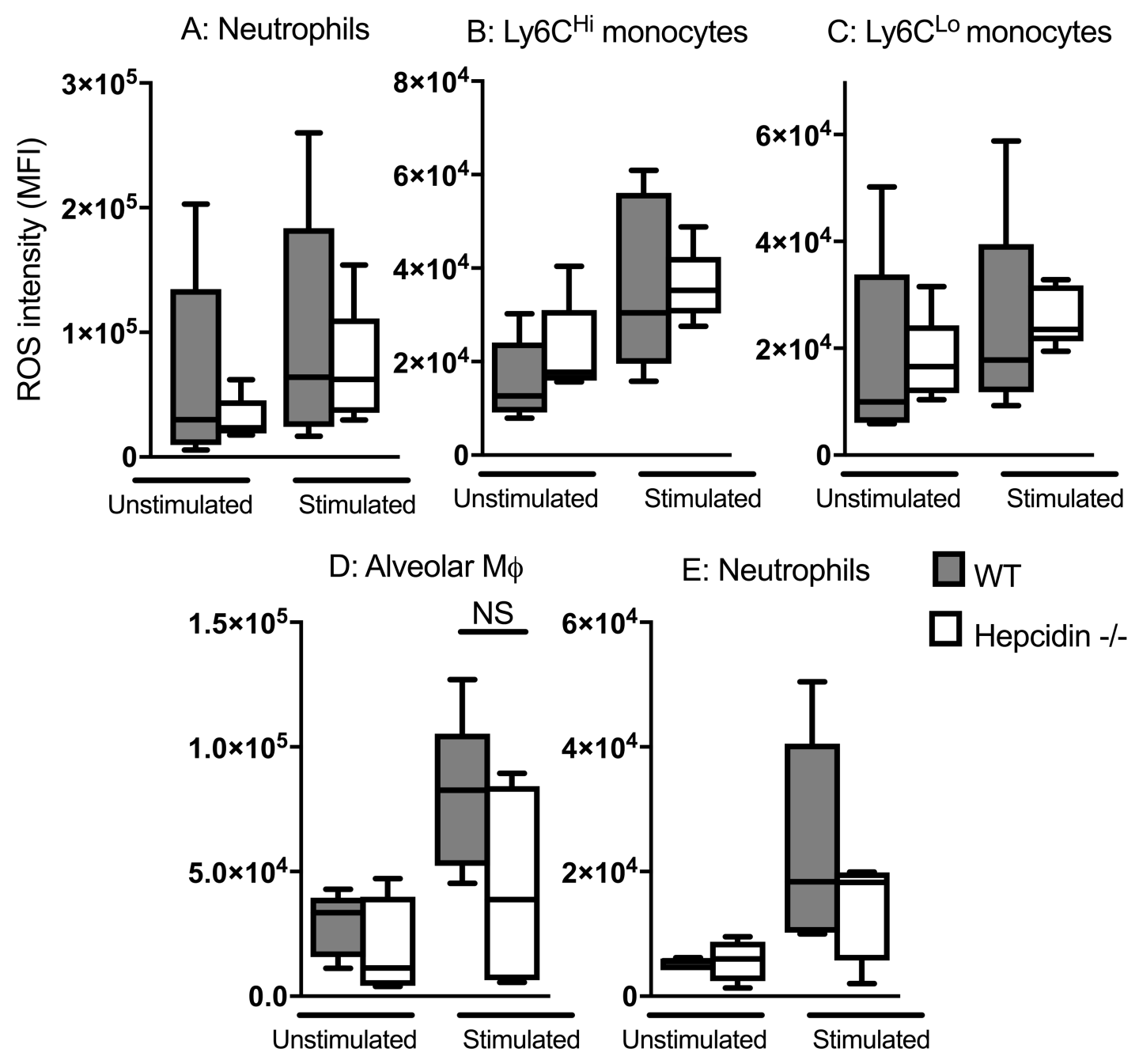


Figure 2.S4. Reactive oxygen species (ROS) production in infected mice. ROS production in indicated cell types from in the blood and bronchoalveloar lavage fluid (BAL) of wildtype and hepcidin-/- mice 2 days following infection. Box and whisker plots show median (line within box), upper and lower quartiles (upper and lower box boundaries), and total range (bars); $n=4-5$ per group; NS, not significant ( $t$-test) for comparing ROS production between WT and hepcidin-/- mice. 


\subsubsection{Restoring hypoferremia is sufficient to protect hepcidin-deficient mice from infection}

Impaired hepcidin production is common in the clinical setting, for example in patients with liver disease ${ }^{156-159}$, and our data suggests a mechanism by which these hosts may be predisposed to Gram-negative bacterial infections. As proof-of-principle of a potential therapeutic approach in this population, we assessed the effect of minihepcidin, a hepcidin analogue, on the outcome of $K$. pneumoniae infection in hepcidindeficient hosts. We used a previously described synthetic peptide based on the $9 \mathrm{~N}$ terminal amino-acids of hepcidin molecule, which are necessary and sufficient to induce the degradation of ferroportin ${ }^{160,161}$. Daily treatment with the hepcidin analogue beginning on the day of infection resulted in markedly lowered plasma iron levels in infected hepcidin-deficient mice as compared to vehicle-treated controls without affecting the total lung iron content (Figure 2.7A-B), consistent with utilization of plasma iron in erythropoiesis ${ }^{4}$. The treatment also resulted in $>99 \%$ reduction in median lung and blood bacterial burden in infected hepcidin-deficient mice and $40 \%$ reduction in mortality compared with vehicle alone (Figure 2.7C-D). These data provide evidence that the acute induction of hypoferremia during infection in hepcidin-deficient hosts may represent a plausible therapeutic strategy. 


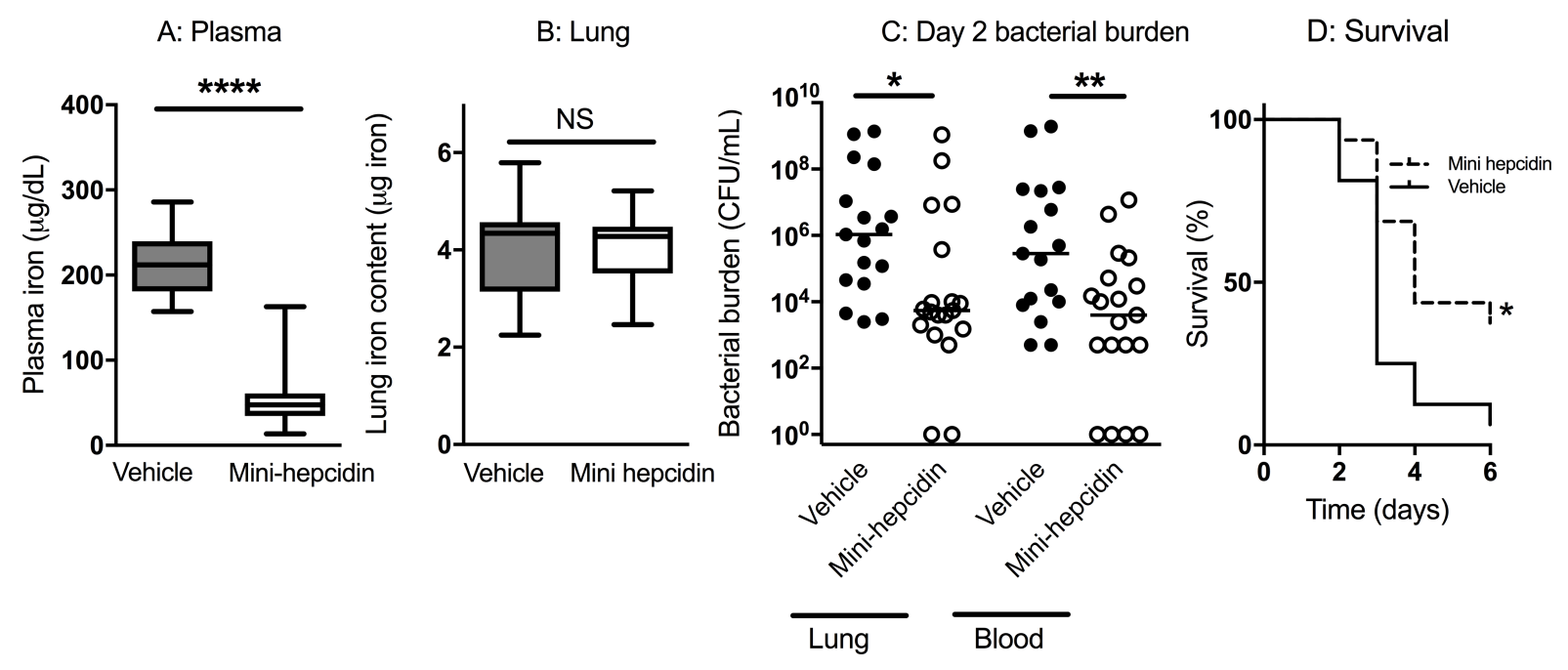


Figure 2.7: The effect of hepcidin agonist treatment in Klebsiella pneumonia in hepcidin-deficient mice. (A-B): Non-heme iron levels in the plasma and lung of vehicle-treated and mini-hepcidin treated hepcidin-deficient mice. Box and whisker plots show median (line within box), upper and lower quartiles (upper and lower box boundaries), and total range (bars). $N=17-19$ per group combined from 2 independent experiments; ${ }^{* \star \star \star}, p<0.001$; NS $=$ not significant by $t$-test. (C): Bacterial burden in blood and lungs. Horizontal lines represent median and each circle represents one animal; animals with no detectable bacteria are reported to have a bacterial burden of 1 colony forming units on the logarithmic scale; $n=17-19$ per group, combined data from 2 experiments; * and ${ }^{* *}$ denote $p$ values $<0.05$ and ${ }^{* *}<0.01$ by Mann-Whitney test, respectively. (D): Survival of hepcidin-deficient mice treated with either vehicle or hepcidin analogue days $0-5 ; n=16$ per group, combined result of 2 experiments; ${ }^{*}, p$ $<0.05$ by Mantel-Cox test. 


\subsubsection{Mini-hepcidin does not suppress plasma iron in wildtype mice nor confer protection}

After we validated that mini hepcidin could restore protection in hepcidin-deficient mice, we next texted the hypothesis that mini hepcidin treatment could protect wildtype mice against infection. Because we observed that hepcidin is not induced until day 2 post-infection in wildtype mice (Figure 2.2A), we reasoned that prophylactic hepcidin treatment may further suppress bacterial growth in the early stages of infection. As previously reported during $V$. vulnificus infection ${ }^{55}$, we found that treatment with mini hepcidin beginning one day before infection did not further suppress plasma iron in wildtype mice (Figure 2.8A). Consistent with our observations in hepcidin-deficient mice, mini-hepcidin treatment did not affect lung tissue iron content (Figure 2.8B). Surprisingly, wildtype mice treated with mini hepcidin had approximately 10 -fold higher lung bacterial burdens as compared to vehicle-treated mice, although no differences were observed in the blood (Figure 2.8C). These results suggest that additional hepcidin does not further suppress plasma iron nor confer a protective benefit in hepcidin-competent hosts. Our unexpected finding that additional hepcidin impairs host defense in the lung is consistent with previous reports that hepcidin has antiinflammatory effects independent of iron regulation ${ }^{76,78}$. 


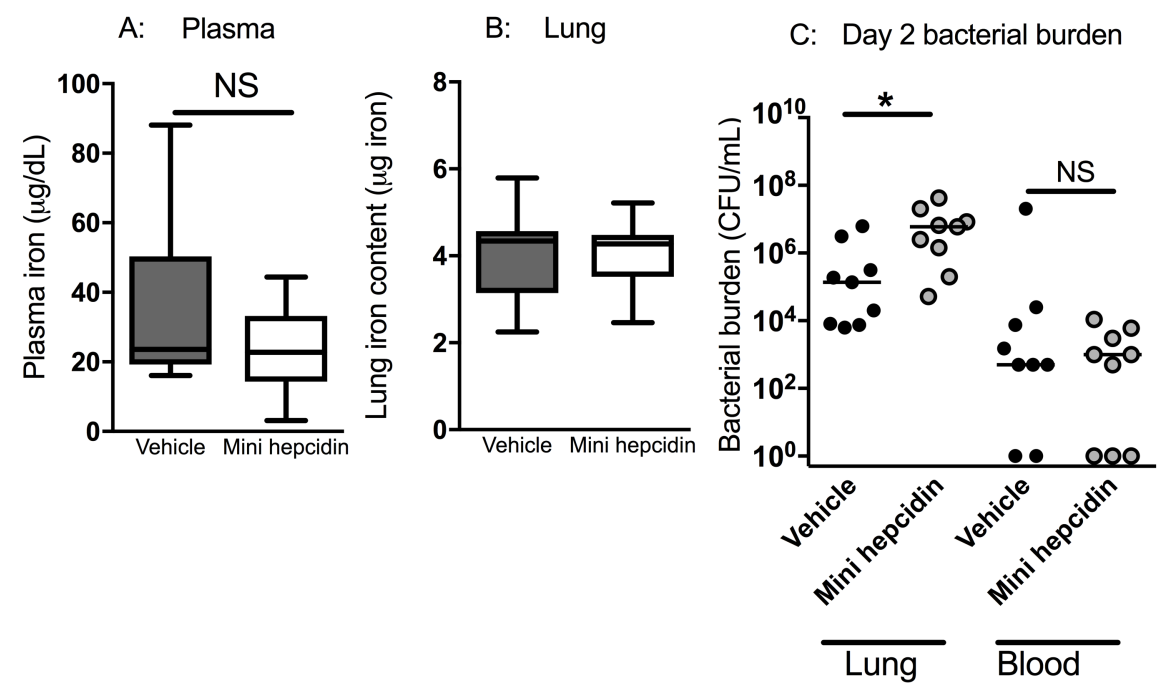


Figure 2.8: The effect of hepcidin agonist treatment in Klebsiella pneumonia in wildtype mice. (A-B): Non-heme iron levels in the plasma and lung of vehicle-treated and mini-hepcidin treated wildtype mice. Box and whisker plots show median (line within box), upper and lower quartiles (upper and lower box boundaries), and total range (bars). $N=0$ per group; NS = not significant by $t$-test. $(C)$ : Bacterial burden in lungs and blood. Horizontal lines represent median and each circle represents one animal; animals with no detectable bacteria are reported to have a bacterial burden of 1 colony forming units on the logarithmic scale; $n=9$ per group, ${ }^{*}$ denotes $p$ values $<0.05$; NS=not significant by Mann-Whitney test, respectively. 


\subsection{Discussion}

Although the competition for iron is thought to be a general feature of antimicrobial host response, the mechanisms and contribution of iron restriction in most infections are undefined. In particular, hepcidin-mediated hypoferremia has only been implicated in host defense against $V$. vulnificus, an organism with specific affinity for iron-overloaded hosts, but its role in defense against common pathogens and in normal hosts is not known ${ }^{144}$. We showed that hepcidin was necessary for protection against bacterial dissemination in Gram-negative pneumonia, and that treatment with iron citrate was sufficient to permit bacterial outgrowth in wildtype mice. Finally, we showed that treatment with mini-hepcidin restored hypoferremia and rescued iron-overloaded, hepcidin-deficient hosts from the infection. Taken together, these data suggest that hepcidin-mediated iron sequestration protects against Gram-negative pneumonia.

Our data suggest that the hepcidin-dependent protection was mediated by reducing plasma iron, as indicated by our observation that wildtype mice treated with iron citrate were susceptible to $K$. pneumoniae infection despite robust hepcidin induction (Figure 2.1E-F), and that hepcidin-deficient mice were protected from infection if starved of iron (Figure 2.S3C). While there may be iron-independent effects of ferric ammonium citrate administration that we have not accounted for, such as bacterial fermentation of citrate, we are encouraged that the phenotype of iron citrate-treated mice is remarkably similar to that of hepcidin-/- mice in terms of death and bacterial burden. Furthermore, we found that hepcidin-deficient mice treated with mini-hepcidin were robustly protected from infection despite having tissue iron overload, suggesting that restriction of iron in the plasma is sufficient for protection (Figure 2.7C-D). Hepcidin 
exhibits antimicrobial activity against a broad array of pathogens in vitro, and an ironindependent direct microbicidal role for hepcidin has thus been suggested ${ }^{7,162,163}$. Arguing against such a direct microbicidal role, we report plasma hepcidin levels at concentrations much lower than those required to mediate killing in vitro ${ }^{162}$. Similarly, administration of a hepcidin analogue, which lacks the beta-defensin motif required for its antimicrobial activity ${ }^{160}$, rescued the phenotype of hepcidin-deficient mice (Figure 2.7 C-D).

While hepcidin can be produced by leukocytes, airway epithelial cells and other tissues $^{26,39,66}$, our results indicate that hepcidin is predominantly produced by the liver and circulates systemically in the context of bacterial pneumonia. Consistent with this, we observed that hepcidin induction was dependent on IL-6, similar to observations with other systemic inflammatory stimuli ${ }^{164}$. In addition, we found that IL-6 neutralization disrupted host defense against $K$. pneumoniae (Figure 2.3A), in corroboration with previous reports ${ }^{148}$. While IL-6 induces many acute phase reactants, such as $C$ reactive protein, which are crucial to the innate immune response, we speculate that hepcidininduction may be a component of IL-6-mediated protection.

Our unexpected findings that mini-hepcidin administration modestly impaired pulmonary defense against Gram-negative pneumonia in wildtype mice suggest several distinct possibilities (Figure 2.8). It has been previously reported that hepcidin protein appears to have anti-inflammatory effects on the immune response, which in the setting of prophylactic administration potentially reduced the ability of mice used in this study to resist pulmonary infectio ${ }^{78}$. The mechanism of iron-independent hepcidin regulation of inflammation was proposed to be a result of activation of Suppression of Cytokine 
Signaling 3 (SOCS 3 ) upon hepcidin-induced degradation of ferroportin in target cells ${ }^{76}$. Recently, a group reported that hepcidin reduced hepatic stellate cell activation in a murine model of liver fibrosis, thereby reducing overall liver pathology. The mechanism of hepcidin-mediated protection was found to be the disruption of ferroportin-dependent Smad3 phosphorylation ${ }^{165}$. Together, these reports suggest that the signaling events downstream of hepcidin-mediated ferroportin degradation influence the immune landscape and are worth further study. 


\subsection{Methods}

\subsubsection{Animals and in vivo procedures}

C57BL/6 mice, purchased from Jackson Laboratories (Bar Harbor, ME), and Hepc1-/- mice generated on a C57BL/6 background ${ }^{152}$, acquired from the University of California, Los Angeles, were bred at the University of Virginia under specific-pathogen free conditions for a minimum of 2 generations and offspring were used in the experiments. To minimize any effect of differing microbiota between groups of animals, all animals were bred and maintained in the same room in the vivarium, and used bedding from cages of breeders and animals to be used in experiments were intermixed at weekly intervals. Age- and sex-matched male and female 6-12 week-old mice were used in experiments. Animal experiments were performed in compliance with $\mathrm{NIH}$ Guide for the Care and Use of Laboratory Animals, The US Animal Welfare Act, PHS Policy on Humane Care and Use of Laboratory Animals and was approved by the Institutional Animals Care and Use Committee at the University of Virginia (protocol \#3571).

Standard rodent chow has artifactually high iron content that causes nearmaximal expression of hepcidin at baseline in wildtype animals ${ }^{164}$. To correct this, all animals were conditioned on a low iron diet (2-4ppm iron; Teklad diet TD.80396, Envigo, Madison, Wisconsin) for 5-7 days prior to, and throughout the duration of, all experiments. In our pilot studies and published reports ${ }^{164,166}$, this protocol normalizes baseline hepcidin expression without inducing iron deficiency. In some experiments, hepcidin-deficient mice were weaned to iron deficient chow at 22 days of age for 3-4 weeks before use in experiments in order to induce iron deficiency. 
Experimental bacterial pneumonia was induced as previously described ${ }^{92,147}$, by intratracheal inoculation of 500-1500 colony-forming units (CFU) of $K$. pneumoniae strain 43816 (American Type Culture Collection, Manassas, Virginia). In some experiments, $1500 \mathrm{CFU}$ of $K$. pneumoniae was delivered intravenously in $100 \mu \mathrm{L}$ PBS. To induce transient hyperferremia without iron overload, $900 \mu \mathrm{g}$ of ferric ammonium citrate in $100 \mu \mathrm{L}$ PBS was delivered intraperitoneally, using a previously characterized protocol $^{167,168}$. In some experiments, mice were given $1 \mathrm{mg}$ anti-IL-6 antibody or isotype control intraperitoneally $3 \mathrm{hr}$ prior to and $24 \mathrm{hr}$ following infection (clones MP5-20F3 and HRPN1, BioXcell, West Lebanon, New Hampshire). In other experiments, $100 \mathrm{nmol}$ of the synthetic hepcidin analogue PR73, referred to as "mini-hepcidin" in text, or vehicle was administered via intraperitoneal injection beginning $5 \mathrm{hr}$ prior to infection and then every 24 hr until day 5 post-infection. PR73 was synthesized using Fmoc solid-phase peptide synthesis, and solubilized for in vivo use with SL220 (a polyethylene glycosylated-phospholipid based solubilzer; NOF America, White Plains, New York) as previously described ${ }^{55,160,161}$.

\subsubsection{Tissue harvest and determination of bacterial burden}

Animals were euthanized with an overdose of a ketamine/xylazine solution, blood was collected from the right ventricle into heparinized syringes and pulmonary vasculature was perfused with $2 \mathrm{~mL}$ of PBS-2mM ethylenediaminetetraacetic acid (EDTA). The median liver lobe was collected for RNA analysis. Lungs and liver were homogenized in $1 \mathrm{~mL}$ PBS. BAL was performed by serially inflating the lungs via an intratracheal catheter with $1 \mathrm{~mL}$ of PBS-2mM EDTA and recovering the fluid 4 times $^{91}$. 
For bacterial enumeration, lung homogenates and blood were serially diluted in sterile water and cultured on blood agar plates, as previously described ${ }^{169}$.

\subsubsection{RNA, protein, and iron analysis}

Tissue RNA was extracted using TRIzol and chloroform (Thermo-Fisher Scientific; Waltham, Massachusetts). Buffy coat RNA was extracted using a commercial kit (Qiagen; Hilden, Germany). A commercial kit was used to synthesize cDNA (Promega; Madison, Wisconsin). Hepcidin transcript was quantified by qRT-PCR in duplicate using SYBR green (Bioline; Luckenwalde, Germany). Expression was calculated using the $\Delta \Delta \mathrm{C}(\mathrm{t})$ method normalized to glyceraldehyde 3-phosphate dehydrogenase, and then relative to hepcidin expression of uninfected animals. Primers were custom-synthesized (Sigma Aldrich, St. Louis, Missouri) as previously published 158: hepcidin 5'TGCAGAAGAGAAGGAAGAGAGACA (forward) and 5'CACACTGGGAATTGTTACAGCATT (reverse); glyceraldehyde 3-phosphate dehydrogenase 5'TTCACCACCATGGAGAAGGC (forward) and 5'GGCATGGACTGTGGTCATGA (Reverse) ${ }^{170}$. The thermal cycling conditions were as follows: 2 min at $95^{\circ} \mathrm{C}, 10$ minutes at $95^{\circ} \mathrm{C},\left[5\right.$ seconds at $95^{\circ} \mathrm{C}, 10$ seconds at $55^{\circ} \mathrm{C}$ ] for 40 cycles. Reactions were performed on a BioRad iQ5 Cycler. Protein concentration of hepcidin was quantified using a commercial ELISA kit (Intrinsic Life Sciences; La Jolla, California) according to the manufacturers' instructions. Plasma iron was quantified with a commercial kit (Sekisui Diagnostics; Lexington, Massachusetts) according to manufacturer instructions with minor modifications, namely samples were scaled to volume and centrifuged at $10,000 \mathrm{~g}$ for 10 minutes between steps to clear insoluble 
material. Transferrin saturation was determined by measuring the unsaturated iron binding capacity (UIBC) of each sample using a commercial kit (Sekisui Diagnostics; Lexington, Massachusetts). UIBC values were added to plasma iron values in each sample to calculate the total iron binding capacity (TIBC). Transferrin saturation was calculated as (plasma iron/TIBC) $\times 100 \%$.

Tissue heme and non-heme iron levels were quantified using the ferrozine assay, as previously described ${ }^{171-173}$. Whole lungs and left lateral lobe of the liver were homogenized in $1 \mathrm{~mL}$ of water. BAL was collected into $1 \mathrm{~mL}$ of PBS-2mM EDTA. Briefly, $100 \mu \mathrm{L}$ sample were heated in $100 \mu \mathrm{L}$ buffer containing $1 \mathrm{~N}$ hydrochloric acid and $10 \%$ trichloroacetic acid with (total iron) or without (non-heme iron) $2.25 \% \mathrm{KMnO}_{4}$ at $60^{\circ} \mathrm{C}$ for 2 hours. Samples were centrifuged at $10,000 \mathrm{rpm}$ for 10 minutes to remove insoluble material and were incubated with $50 \mu \mathrm{L}$ iron detection reagent $(6.5 \mathrm{mM}$ ferrozine, $6.5 \mathrm{mM}$ neucoproine, $1 \mathrm{M} \mathrm{L}$ ascorbic acid, $2.5 \mathrm{M}$ ammonium acetate) for 10 minutes and then plated in duplicate and read at $A_{590} \mathrm{~nm}$ on a Dynex Triad microplate reader (Dynex Technologies, Chantilly, Virginia). Heme-iron values were calculated by subtracting non-heme iron values from total iron content. Labile plasma iron (LPI), the major component of non-transferrin-bound iron, was measured using a published protocol with minor modifications ${ }^{174}$ : briefly, HEPES buffered saline was iron-depleted by incubation with Chelex-100 chelating resin (Bio-Rad, Hercules, California) for $1 \mathrm{~h}$, filtered, and supplemented with $60 \mathrm{nM}$ deferrioxamine (Sigma). For LPI measurements, $10 \mu \mathrm{l}$ of plasma samples were mixed with either $140 \mu \mathrm{l}$ of $\mathrm{HBS} / 50 \mu \mathrm{M}$ dihydrorhodamine/40 $\mu \mathrm{M}$ ascorbic acid/0.5 mM nitriloacetic acid (“-DFP”), or $140 \mu \mathrm{l}$ of the same buffer supplemented with $100 \mu \mathrm{M}$ deferriprone ("+DFP"). Changes in 
fluorescence were measured over $40 \mathrm{~min}$ and the slope calculated for each sample. The difference between "-DFP" and "+DFP" was used as a measure of LPI. The experiment was repeated a total of three times using different plasma donors with similar results.

Plasma AST and ALT were measured using commercial kits according to the manufacturer instructions (Liquid AST Reagent set and Liquid ALT Reagent Set; Pointe Scientific, Canton, MI).

\subsubsection{Flow cytometry}

Cell suspensions from blood and BAL fluid were prepared and analyzed by flow cytometry as previously described, with minor modifications ${ }^{92,175,176}$. The following antibodies were used for surface antigen staining (from BD Biosciences, San Jose, CA; eBiosciences, San Diego, CA; or Biolegend, San Diego, CA): anti-CD11b Pe-Cy7 (clone M170); anti-CD11c PerCP (clone HL3); anti-CD16/CD32 without fluorochrome (clone 2.4G2); anti-CD45 AmCyan (clone 30-F11); anti-CD64 brilliant violet 421 (clone X545/7.1); anti-CD115 APC (clone AF598); anti-Ly6G PE (clone 1A8); and anti-MHC-II APC (clone M5/114.15.2). After extracellular staining, cells were washed in $1 \%$ fetal calf serum in PBS once, and resuspended in the same buffer. Data were acquired on a FACS Canto II instrument using BD FACS Diva software (version 8.0; BD Biosciences) and analyzed with FlowJo software (version 8.8.6; Tree Star, Ashland, Oregon). The absolute cell number was calculated as the product of the cell type frequency (as determined by gating analysis) and the total number of cells in the sample (as determined by manually counting under a hemocytometer). 
After extracellular staining, cells were washed in FA buffer once and prepared for reactive oxygen species staining using DCFDA (2',7'-dichlorofluorescein diacetate) with a commercial kit (Abcam, Cambridge, UK) according to the manufacturer's instructions. Cells were stained using $2 \mu \mathrm{M}$ DCDFA for 15 minutes at $37^{\circ} \mathrm{C}$. TBHP (Tert-butylhydrogen peroxide) was added to a final concentration of $50 \mu \mathrm{M}$ for stimulated cells. Cells were incubated for an additional 45 minutes at $37^{\circ} \mathrm{C}$. Data were acquired immediately after incubation on a FACS Canto II instrument using BD FACS Diva software (version 8.0; BD Biosciences) and analyzed with FlowJo software (version 8.8.6; Tree Star, Ashland, Oregon).

\subsubsection{In vitro experiments}

Bone marrow neutrophils were isolated by grinding the tibia, femur, pelvis, and spine from uninfected mice in a sterile mortar and pestle as previously described ${ }^{147,169}$ followed by positive selection using anti-Ly6G magnetic beads, according to the manufacturer's instructions (MACS; Miltenyi Biotec, Bergisch Gladbach, Germany). Isolated populations were found to have $>90 \%$ viability by examination in trypan blue. Neutrophil bacterial killing was quantified using a published with minor modifications ${ }^{169}$. Briefly, K. pneumoniae was opsonized at a concentration of $8 \times 10^{6} \mathrm{CFU} / \mathrm{mL}$ in $20 \%$ fresh mouse serum and Hank's balanced salt solution (HBSS) for 30 minutes at $37^{\circ} \mathrm{C}$, washed once in cold water, once in HBSS, and re-suspended in HBSS. Aliquots of $2 \times 10^{5}$ bacteria were added to sterile U-bottom 96-well plates and co-incubated with $5 \times 10^{5}, 2 \times 10^{5}, 2 \times 10^{4}, 2 \times 10^{3}$, or no neutrophils from wildtype or hepcidin-deficient mice in triplicate in a total volume of $200 \mu \mathrm{L}$ HBSS. Samples were incubated in a humidified 
chamber with $5 \% \mathrm{CO}_{2}$ at $37^{\circ} \mathrm{C}$ for 1 hour. Number of surviving bacteria was determined by hypotonic lysis of neutrophils in cold water and serial dilution and culture.

Human plasma was inactivated $\left(30 \mathrm{~min}\right.$ at $\left.56^{\circ} \mathrm{C}\right)$ and incubated overnight at $37^{\circ} \mathrm{C}$ with $0-60 \mu \mathrm{M}$ ferric ammonium citrate, followed by quantification of transferrin saturation and LPI. The samples were then used to generate $90 \%$ plasma agar solid media, poured in sterile 12-well tissue culture plates. K. pneumoniae was suspended at $10^{4}$ $\mathrm{CFU} / \mathrm{ml}$ and $5 \mu \mathrm{l}$ plated in triplicates on plasma agar wells, incubated for $24 \mathrm{hr}$ at $37^{\circ} \mathrm{C}$ and bacterial colonies were counted and photographed.

\subsubsection{Statistics}

Data were analyzed using Prism software (version 6.0, GraphPad Software, San Diego, California). The Mantel-Cox test was used to analyze survival data. A one-way ANOVA with a post-test for linear trend was used to analyze change in a single parameter over time, and one-way ANOVA with a Tukey multiple comparison post-test was used to analyze differences between 3 or more groups. A two-way ANOVA with Tukey multiple comparison post-test was used to compare changes over time between 2 groups. An unpaired, 2-tailed $t$-test was used to compare 2 groups for RNA, protein and iron levels. A Mann-Whitney test was used to compare bacterial burden between groups. A $p$ value of $<0.05$ was considered statistically significant. 


\section{Chapter III: The role of heme uptake in invasive pulmonary aspergillosis}

\subsection{Introduction}

Invasive pulmonary aspergillosis is a severe lung infection caused by the saprophytic fungus Aspergillus, and is a significant cause of morbidity and death in immunocompromised patients ${ }^{107}$. Although triazoles have improved outcomes in invasive pulmonary aspergillosis, mortality remains at $30-50 \%$, depending on the patient group $^{110}$. It is therefore warranted to explore and identify new therapeutic strategies for treating infection.

As discussed in chapter I, iron acquisition is a crucial component of microbial pathogenesis. Pathogenic bacteria and fungi have numerous ways to import iron in the host setting, such as direct import, siderophore-mediated scavenging, or uptake of host iron-containing molecules such as transferrin ${ }^{98,142,177}$. In this context, importing extracellular heme or hemoglobin is mechanistically distinct from ferric iron import, because heme-iron is covalently bound to a protoporphyrin ring and cannot be chelated by iron scavengers. For example, Haemophilus influenzae requires exogenous heme for growth and can utilize heme-ablumin, heme-hemopexin, hemoglobin, and hemoglobin-haptoglobin using at least 3 separate transporters with overlapping roles in virulence during infection ${ }^{178-180}$. Heme transporters contribute to Staphylococcus aureus virulence during systemic infection, even if other iron acquisition mechanisms are left intact $^{181}$. Numerous bacterial and fungal pathogens encode heme and hemoglobin transporters that are important for growth in different conditions in vitro, but have uncertain roles in vivo, including Helicobacter pylori, Candida albicans, Histoplasma capsulatum, and Cryptococcus neoformans ${ }^{182-185}$. 
The host can potentially counter microbial hemoglobin and heme uptake by producing the scavengers haptoglobin and hemopexin. Haptoglobin is an abundant protein in normal serum that tightly binds hemoglobin and neutralizes its oxidative potential ${ }^{186}$. Haptoglobin-hemoglobin complexes are cleared via the tissue macrophage scavenger receptor $C D 163^{187}$. Hemopexin is an inducible glycoprotein that serves as a second-line defense against the release of cell-free hemoglobin. Hemopexin binds heme to neutralize oxidative activity and targets it for uptake via the low density cholesterol receptor (also known as CD91), which is expressed on tissue macrophages, hepatocytes, and monocyte-lineage cells ${ }^{188}$. While both proteins are positively induced acute phase reactants, hemolytic disorders can result in depletion of haptoglobin and hemopexin from the blood ${ }^{186}$. Haptoglobin and hemopexin are indispensable for protecting the host against vascular damage caused by hemolysis; due to its reactive nature, heme released during hemolysis will promote further erythrocyte destruction unless cleared from circulation ${ }^{189,190}$. Engagement of the hemopexin-heme complex with CD91 on monocytes further dampens inflammatory responses ${ }^{191}$. The role of haptoglobin and hemopexin-mediated clearance of heme species is mostly unknown and untested in the context of infection. The most definitive report showing this mechanism is important during infection was published recently, where the authors showed that hemopexin is critically important for restricting bacterial growth in a murine Citrobacter rodentium model ${ }^{192}$. Another group showed that hemopexin was essential to protect against polymicrobial sepsis, where the mechanism of protection was suppression of vascular injury rather than inhibition of microbial growth ${ }^{193}$. 
As discussed in Chapter I, iron acquisition is an essential component of invasive pulmonary aspergillosis pathogenesis: siderophores are required for virulence in Aspergillus species, and host iron overload is an independent risk factor for disease in immunosuppressed patients ${ }^{124,131}$. Heme is the largest potential iron source in the context of host infection, as $\sim 70 \%$ of all iron within mammals is hemoglobin-associated. It is controversial whether Aspergillus species have access to heme during infection: while most authors within the field of fungal pathogenesis state that Aspergillus species possess no mechanisms to import heme, several reports from the industrial microbiology field suggest that Aspergillus species can utilize exogenous heme under certain culture conditions via an unknown mechanism ${ }^{120,137}$. Recently, a research group identified a small-molecule inhibitor of fungal heme synthesis which reduced Aspergillus virulence in a Drosophila melanogaster infection model, demonstrating that heme metabolism is a valid therapeutic target ${ }^{194}$. We therefore hypothesized that heme release plays a role in the pathogenesis of invasive pulmonary aspergillosis.

\subsection{Results}

\subsubsection{Infection with $A$. fumigatus induces pulmonary hemorrhage}

We began by depleting mice of neutrophils using an anti-Ly6G antibody, and instilling A. fumigatus conidia directly into the trachea of anaesthetized mice the following day. We first sought to characterize the effects of infection on iron metabolism in mice. We hypothesized that, like many infections, mice infected with $A$. fumigatus induce hepcidin and develop hypoferremia, which could play a role in iron restriction during infection. Surprisingly, we found that although hepcidin was induced during 
infection (Figure 3.1A), mice did not develop hypoferremia and instead had constant iron levels in the plasma (Figure 3.1B). We further observed that plasma collected from infected mice was tinted red, and measured the hemin content in a small subset of mice. There was a trend towards increased hemin content in the plasma following infection, while not significant possibly due to low sample size, that was suggestive of hemolysis, a process that could potentially negate hepcidin-mediated hypoferremia (Figure 3.1C). We found that mice infected with $A$. fumigatus developed pulmonary hemorrhage, quantified by a distinct increase in total hemoglobin (Figure 3.1D). Because hemoglobin is a rich source of iron, we next sought to characterize how iron available in red blood cells might be available to A. fumigatus during the infection. We thus measured the amount of cell-free heme and non-heme iron recovered from the cell-free fluid of a BAL in infected mice to characterize if iron was being released into the extracellular space of the airways, where it is potentially accessible to A. fumigatus. Heme-associated iron, but not non-heme associated iron, increased following infection, suggesting that hemolysis was occurring the lungs of infected mice (Figure 3.1E). We examined histology sections from infected mice to better understand the cause of pulmonary hemorrhage, and found, as others have reported, that germinating $A$. fumigatus hyphae sometimes penetrate major blood vessels in the lung, releasing blood into the alveolar space (Figure 3.1F $)^{195}$. We therefore reasoned that heme represented a potential iron source for $A$. fumigatus growing in the lungs of mice. 

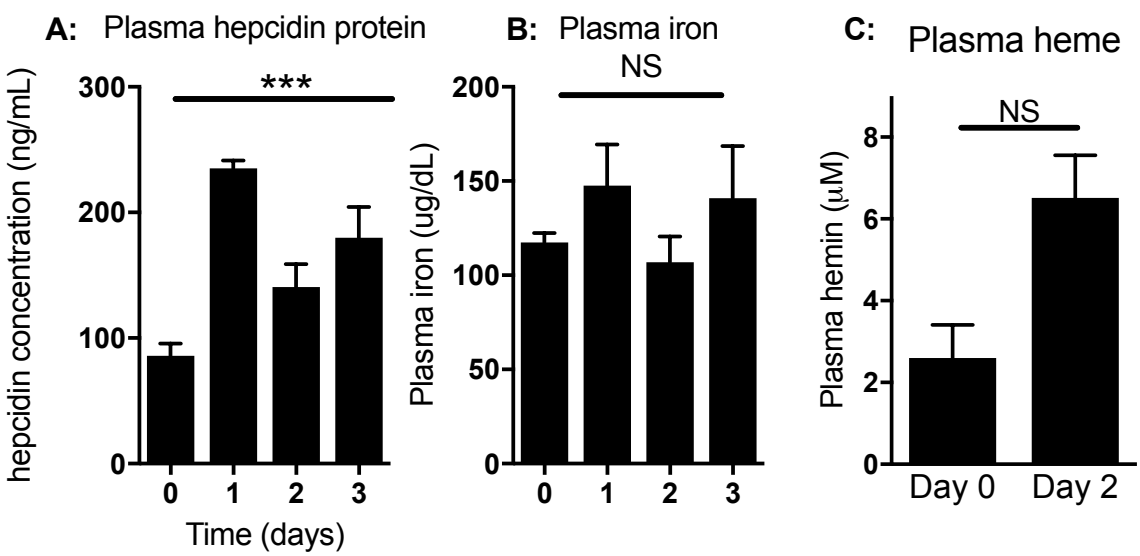

D: Lung hemoglobin

E:

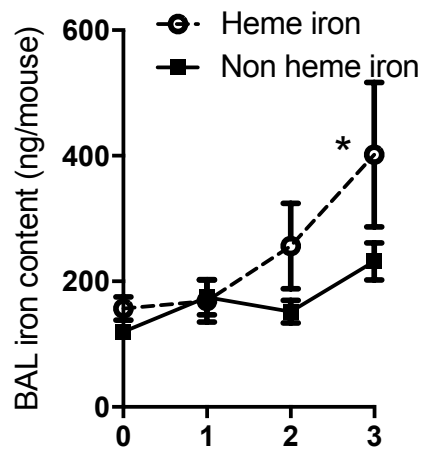

F:
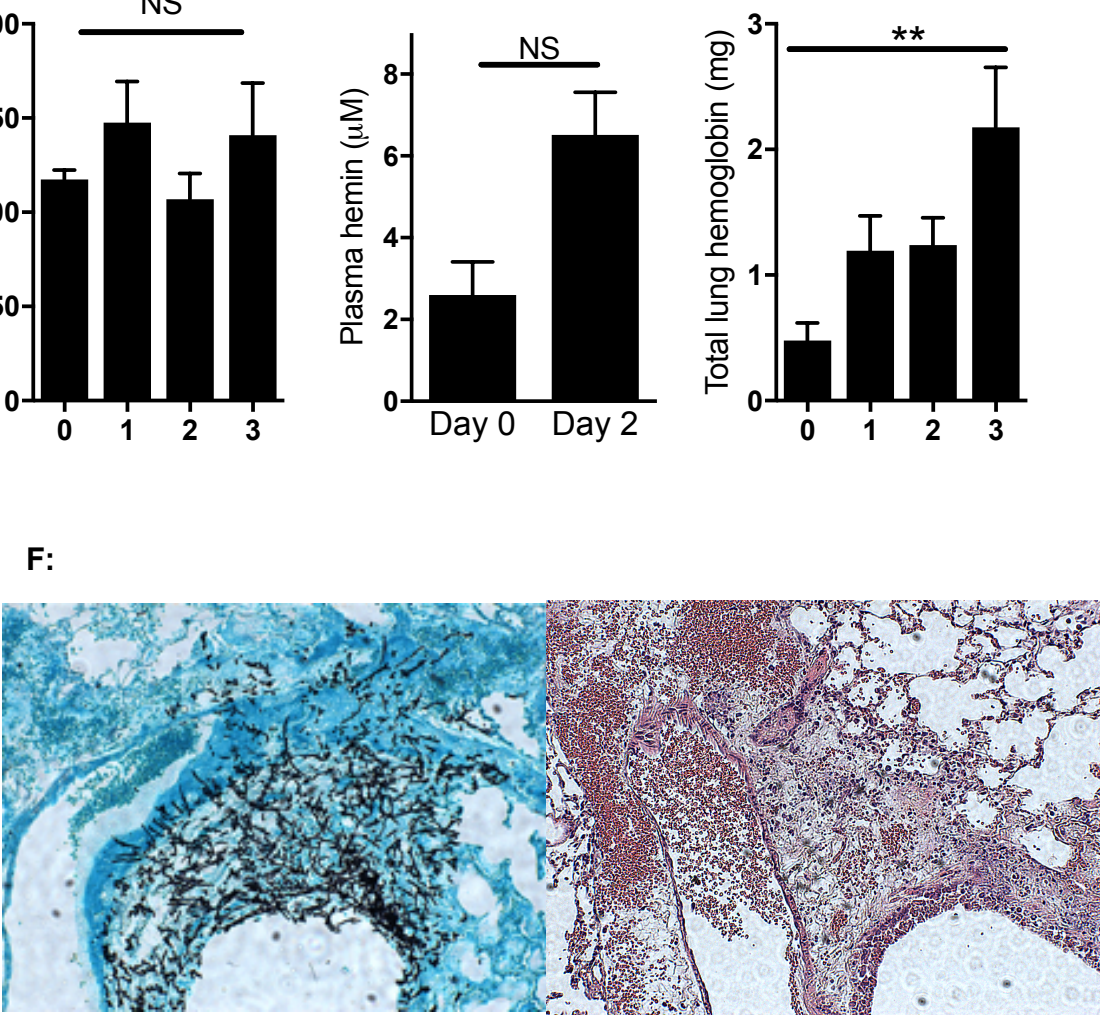
Figure 3.1. Pulmonary hemorrhage during invasive pulmonary aspergillosis. Neutropenic WT mice were infected intratracheally with $A$. fumigatus conidia and monitored for 3 days. A: Plasma hepcidin protein, $N=4-5$ per time point; $B$ : Plasma iron, $\mathrm{N}=8-10$ per time point; C: Plasma hemin concentration, $\mathrm{N}=3-7$ per group; $\mathrm{D}$ : hemoglobin protein in whole lung homogenate, $\mathrm{N}=10$ per time point. Bars =mean+/-SEM. NS, ${ }^{* *}$, *** denote not significant, $\mathrm{P}<0.01$, and $\mathrm{P}<0.001$ respectively, one way ANOVA post test for linear trend. E: Acellular heme and non-heme iron recovered from $1 \mathrm{~mL} B A L$ wash. $\mathrm{N}=5-6$ per group, ${ }^{*} \mathrm{P}<0.05$ two way ANOVA. $\mathrm{F}$ : GMS staining of hyphal elements and $\mathrm{H}+\mathrm{E}$ staining of lung structures day 2 post-infection in wildtype mice. 


\subsubsection{A. fumigatus can utilize exogenous heme}

Because there are conflicting reports in the literature as to whether $A$. fumigatus is able to utilize heme, we next tested the growth of $A$. fumigatus in iron-deplete minimal media (AMM) supplemented with various iron sources. As a control, we used Sn(IV) protoporphyrin (PPIX), a heme analog that is naturally fluorescent and consists of a tin ion covalently linked to the center of the protoporphyrin ring instead of an iron ion. We chose to use PPIX in order to control for the extra carbon of the protoporphyrin ring added to low-nutrient media, and because PPIX fluorescence is easily visualized and measured spectrophotometrically. We found while hemin (heme containing ferric iron) supplementation enhanced the growth of $A$. fumigatus in liquid media. A. fumigatus cultures supplemented with PPIX, hemin and ferric chloride displayed thicker hyphal growth compared to AMM alone, and observed enhanced conidiation if cultures were supplemented with ferric chloride or hemin as compared to no supplementation, or PPIX (Figure 3.2A). The most striking difference between the two groups was the greatly enhanced conidiation in cultures that were supplemented with hemin and ferric chloride (Figure 3.2B). While the addition of PPIX did not promote conidiation, it did enhance growth similarly to hemin, suggesting that PPIX may act as a carbon source. In order to better understand how hemin and iron might enhance $A$. fumigatus growth, we also measured the expression of HapX, a transcription factor that mediates response to iron starvation, in A. fumigatus grown under different conditions. We found that both ferric chloride and hemin suppressed HapX expression in A. fumigatus, suggesting that iron in exogenous heme is available to $A$. fumigatus. 
A:

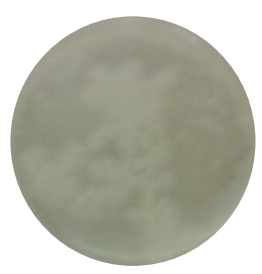

AMM

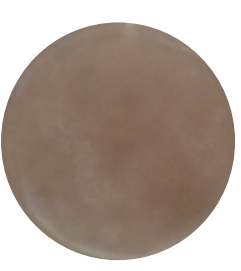

$50 \mu \mathrm{M}$ PPIX

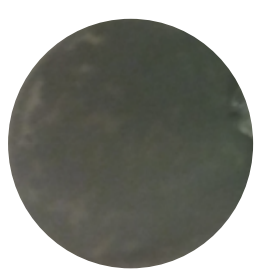

$50 \mu \mathrm{M}$ hemin

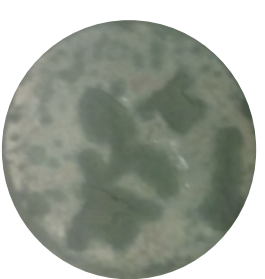

$50 \mu \mathrm{M} \mathrm{FeCl}_{3} \quad 50 \mu \mathrm{M} \mathrm{FeCl}_{3}$ and PPIX
B:

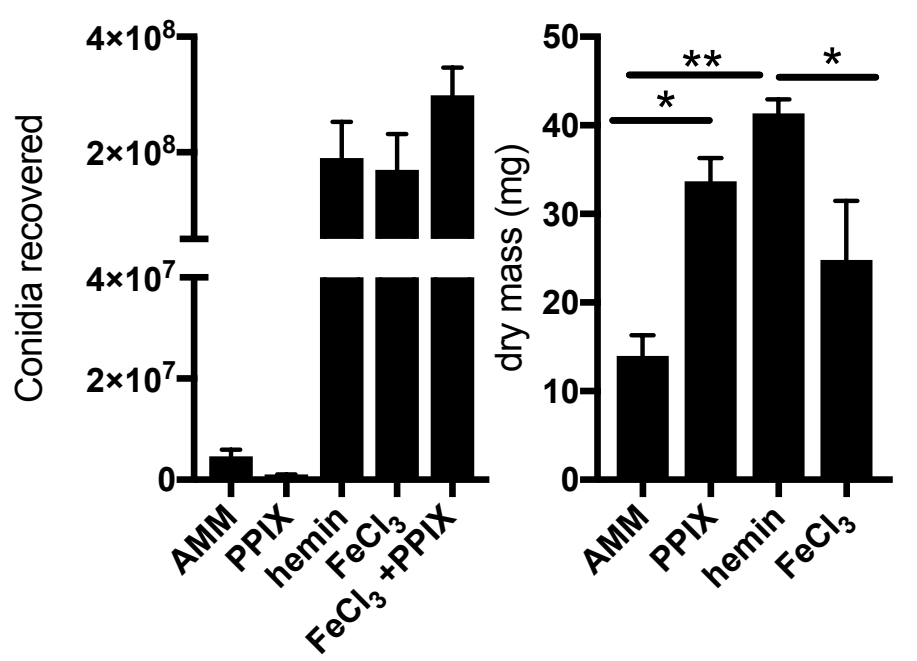

D: HapX expression

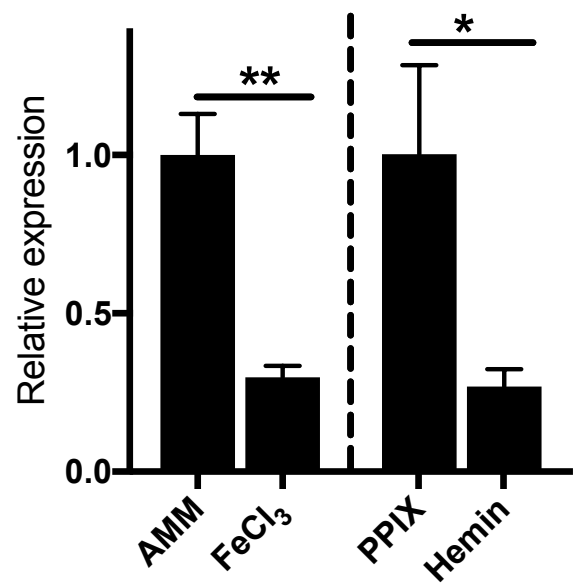


Figure 3.2. Uptake of heme and tin PPIX in vitro. A. fumigatus conidia were grown in iron-free aspergillus minimal media (AMM) alone or with $50 \mu \mathrm{M}$ of indicated supplement: Sn(IV) protoporphyrin (PPIX), hemin, or ferric chloride. A: Hyphal mats of conidia cultured in indicated conditions, representative of 6 . Hyphal elements appear as white tissue, where conidia are blue/grey or green. Pink color is from PPIX. B: Conidia recovered after 4 days of growth in indicated conditions $N=3$. C: Dry mass of hyphal mats grown for 2 days in indicated conditions. $\mathrm{N}=5-6$ per group, ${ }^{*}$ and ${ }^{* *}$ denote $\mathrm{P}$ values of 0.05 and 0.01 respectively, one-way ANOVA. D: Expression of HapX normalized to $\beta$-actin in A. fumigatus grown for 2 days in indicated media. Due to differences in carbon availability, conidia grown in $\mathrm{FeCl}_{3}$ were normalized to $\mathrm{AMM}$, and conidia grown in heme were normalized to PPIX. $N=6,{ }^{*}$ and ${ }^{* *}$ denote $\mathrm{P}$ values of 0.05 and 0.01 respectively, t-test. 
Since hemin enhanced the growth and conidiation of $A$. fumigatus, it was not clear if hemin was imported intact intracellularly, we next assessed for its uptake by the fungus. In order to test this, we cultured A. fumigatus in AMM or AMM containing PPIX and measured fungal fluorescence as a measure of PPIX accumulation. We found that A. fumigatus grown in PPIX, but not AMM, had bright red fluorescence (Figure 3.3A), quantified as an 8-fold higher fluorescence when the organism was incubated with PPIX as compared to AMM or hemin (Figure 3.3B). These results indicate that the intact protoporphyrin ring can associate with $A$. fumigatus hyphae. We observed much weaker staining in germlings (data not shown). To assess whether the fluorescence represented PPIX uptake or association with the cell wall, we assayed the cytosolic fractions of A. fumigatus grown in various media for PPIX fluorescence, and found an 8fold higher fluorescence associated with the cell wall fraction, and a 10-fold higher fluorescence in the cytosolic fraction in A. fumigatus incubated in PPIX (Figure 3.3C). Consistent with this, A. fumigatus hyphae incubated with hemin, but not AMM or PPIX, accumulated intracellular hemin (Figure 3.3D). Together, these results suggest that $A$. fumigatus is able to import at least a portion of exogenous heme, and this is a potential iron source during in vivo infection.

In order to better understand what role heme uptake might play during infection, we tested the hypothesis that heme import influences fungal resistance to oxidative stress. We found that conidia harvested from hyphae cultured on hemin displayed enhanced survival following exposure to hydrogen peroxide as compared to conidia harvested from minimal media plates (Figure 3.3E). Surprisingly, we found that 
germlings grown in the presence of hemin were more susceptible to killing by hydrogen peroxide (Figure 3.3F).

\subsubsection{Exogenous hemin promotes severe infection}

We next investigated whether heme uptake is relevant to the pathogenesis of invasive pulmonary aspergillosis in vivo. We infected neutropenic mice with $A$. fumigatus, as previously described, and coinstilled PBS, $100 \mu \mathrm{g}$ sterile hemin, or a molar equivalent of Sn(IV) PPIX as a control. We selected this dose because the amount of hemin administered is equivalent to the amount contained in $12-14 \mu \mathrm{L}$ whole mouse blood, which is a physiologically relevant dose as it represents $\sim 1.2 \%$ of total mouse blood volume. We found that treatment with hemin, but not PBS or PPIX, worsened infection outcome and led to death in $100 \%$ of infected mice (Figure 3.4A). Uninfected neutrophil-depleted mice treated with hemin all survived. These results suggest that exogenous heme enhances susceptibility to infection in an iron-dependent manner. Since we have previously found that the gut microbiota have a dramatic effect on the type of immune response elicited against $A$. fumigatus ${ }^{196}$, we repeated the experiment using wildtype mice bred at Jackson laboratories and found similar results, suggesting the phenotype is not unique to mice bred at the University of Virginia. (Figure 3.4B). Plasma hemopexin was elevated in mice with invasive pulmonary aspergillosis, but was depleted in mice that were treated with heme (Figure $3.4 \mathrm{C}$ ). We hypothesized that exogenous heme resulted in a more severe infection by serving as a nutrient source for $A$. fumigatus, and examined histology sections collected from UVAbred infected mice one day after infection, before a large portion of hemin-treated mice 
began to die. Interestingly, while mice treated with hemin had more severe pneumonia than mice treated with PBS, there was little germination of $A$. fumigatus conidia in the lungs of either group (Figure 3.4D). We found a similar phenotype in mice bred at Jackson laboratories (data not shown). These results initially suggested that increased A. fumigatus growth was not the mechanism of susceptibility in hemin-treated mice.

We next sought to quantify the fungal burden in mice treated with hemin as compared to mice treated with PBS two days post infection. We measured lung chitin content to determine fungal biomass in infected mice, but were able to measure detectable levels of chitin in only one mouse from either group (Figure 3.5A). Since the chitin assay is known to be insensitive, we used other techniques to quantify $A$. fumigatus growth in lung tissue. We found similar $A$. fumigatus DNA copies in hemin and PBS treated mice as well as mice that were not depleted of neutrophils, with the exception of the one mouse with detectable chitin, suggesting that we were detecting no growth beyond the initial inoculum (Figure 3.5B). Similar results were found when we measured CFU on the same samples (Figure 3.5C). Based on this data, we concluded that enhanced $A$. fumigatus hyphal growth was not the mechanism of heme-mediated susceptibility to infection.

We next hypothesized that excess heme worsened infection by interacting synergistically with $A$. fumigatus antigens to generate fatal immunopathology. In order to test this hypothesis, we challenged neutropenic mice with ethanol-killed $A$. fumigatus germlings coinstilled with PBS or hemin and analysed the subsequent inflammatory response. We found that in contrast to neutropenic mice treated with hemin and live $A$. fumigatus, mice treated with ethanol-killed $A$. fumigatus survived. Mice treated with PBS 
or hemin and killed A. fumigatus germlings had similar concentrations of albumin, TNF, and total protein in the BAL, arguing against antigen and hemin alone as a lethal combination (Figure 3.6 A-C). These results suggest that live A. fumigatus is required to mediate the phenotype. 
A:
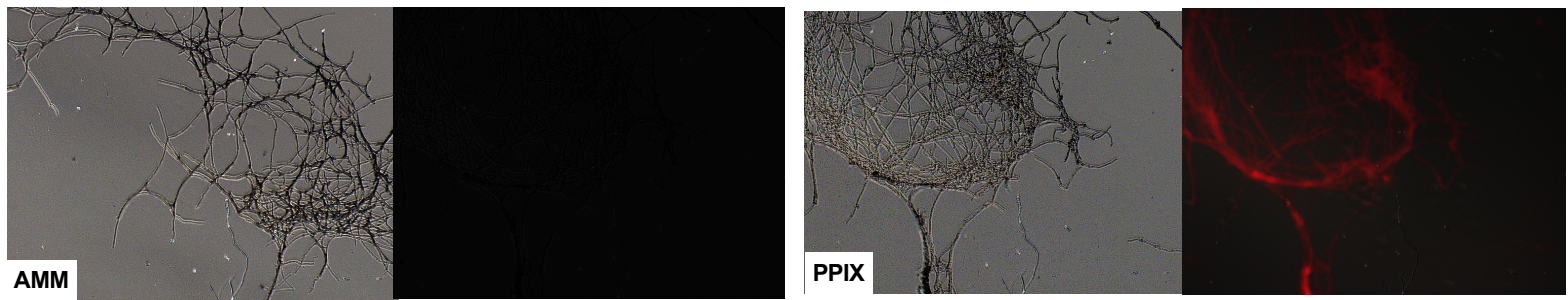

B: PPIX accumulation

C: PPIX localization

D: Heme-iron accumulation

E: Conidia resistance to $\mathrm{H}_{2} \mathrm{O}_{2}$

F: Hyphal resistance to $\mathrm{H}_{2} \mathrm{O}_{2}$
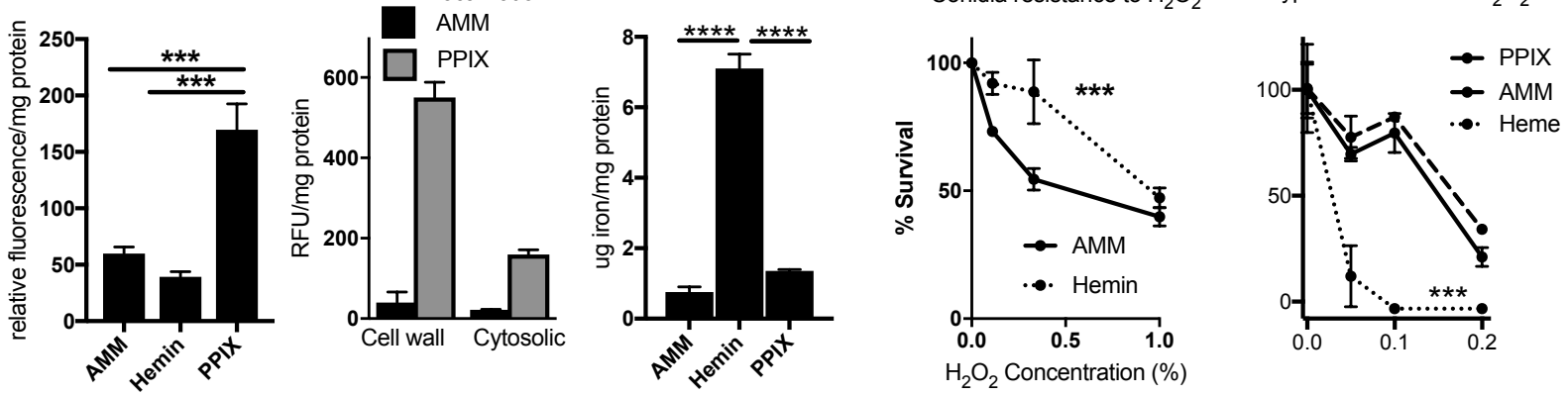
Figure 3.3. Accumulation and utilization of heme in vitro. A. fumigatus conidia were grown in iron-free aspergillus minimal media (AMM) alone or with $50 \mu \mathrm{M}$ of indicated supplement: Sn(IV) protoporphyrin (PPIX), hemin, or ferric chloride. A: Brightfield and fluorescent images of $A$. fumigatus hyphae grown in AMM or $50 \mu \mathrm{M}$ PPIX. B: Fluorescence at excitation/emission of $425 / 590 \mathrm{~nm}$ normalized to total protein recovered from the cytosolic fraction of $A$. fumigatus grown for 2 days in indicated media. $\mathrm{N}=6$ per group, ${ }^{* * *} \mathrm{P}<0.001$, one way ANOVA Tukey test. $\mathrm{C}$ : The cell wall fractions from Figure 3.3B were boiled for 1 hour in SDS. The supernatant was assayed for PPIX fluorescence at $425 / 590 \mathrm{~nm}$ excitation/emission. The cytosolic fraction from Figure $3.3 \mathrm{~B}$ is reproduced to permit direct comparison. D: Heme and non-heme iron recovered from the cytosolic fraction normalized to total protein recovered from the cytosolic fraction of $A$. fumigatus grown for 2 days in indicated media. * and ${ }^{* * *}$ denote $\mathrm{P}$ values of 0.05 and 0.001 respectively, one-way ANOVA Tukey test. E: Survival of conidia following exposure to hydrogen peroxide. E: A. fumigatus cultured for 2 weeks on $\mathrm{AMM}$ or $50 \mathrm{mg} / \mathrm{L}$ hemin. F: Hyphal viability following exposure to hydrogen peroxide. Representative of 2 independent experiments; $\mathrm{N}=3,{ }^{* * *} \mathrm{P}<0.001$ Two-way ANOVA column factor. 
A: UVA mice

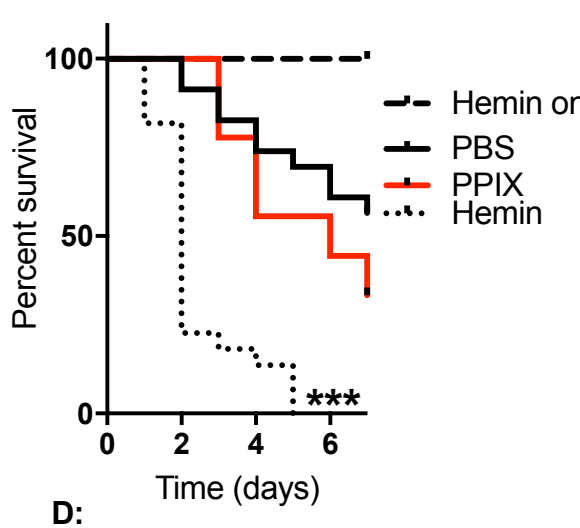

B: Jackson mice

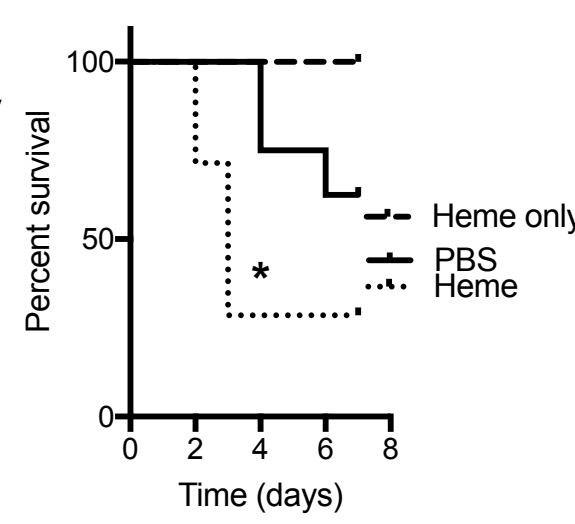

C:

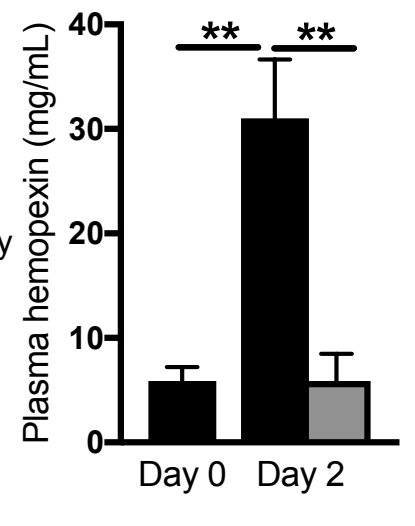

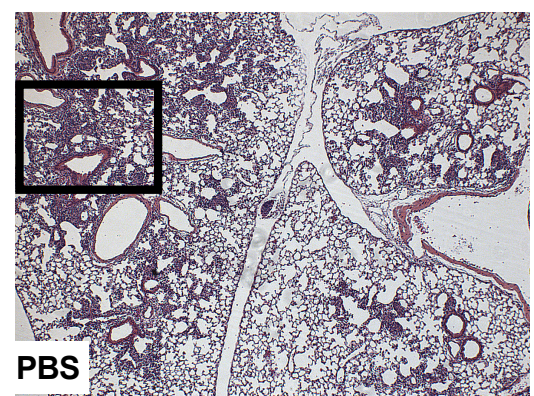
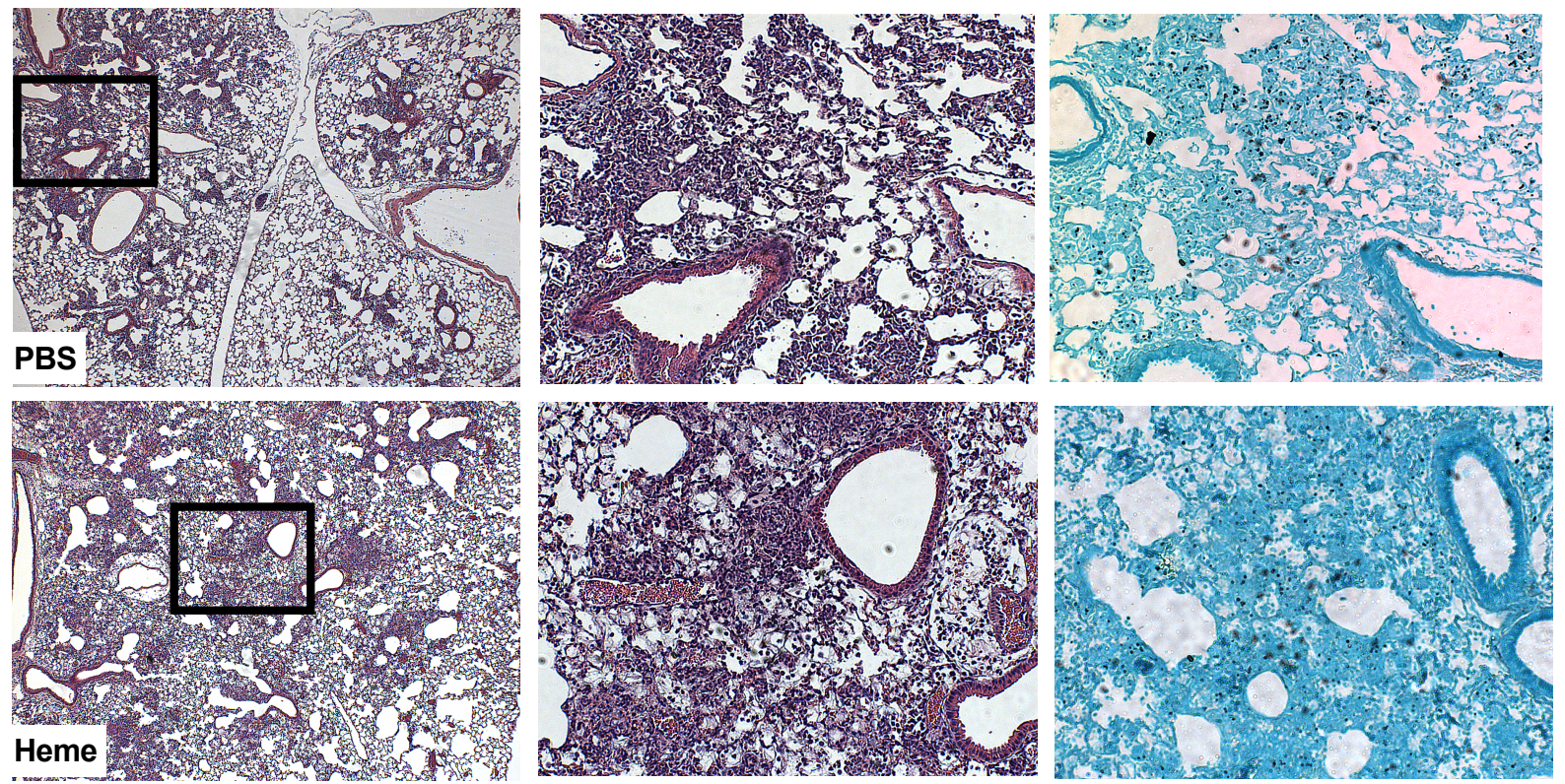
Figure 3.4. The effect of exogenous hemin on invasive pulmonary aspergillosis. A: Survival of neutropenic WT mice bred at UVA treated with hemin only, or $A$. fumigatus conidia coinstilled with hemin, PBS, or Sn(IV)PPIX (PPIX). N=6-17 per group, combined result of two experiments; ${ }^{* * *} \mathrm{P}<0.001$ Log-Rank test. B: Survival of neutropenic WT mice bred at Jackson treated with hemin only, or A. fumigatus conidia coinstilled with hemin or PBS. ${ }^{*} \mathrm{P}<0.05$ Grehan-Breslow-Wilcoxon test. C: Plasma hemopexin in hemin-treated and PBS-treated mice 2 days post infection. $\mathrm{N}=4-5$ per group, ${ }^{* *} \mathrm{P}<0.01$, One way ANOVA Tukey post test. $\mathrm{D}$ : $\mathrm{H}+\mathrm{E}$ and $\mathrm{GMS}$ staining of lung tissue 1 day post infection of mice treated with PBS or hemin. Representative slide of 34 mice. 


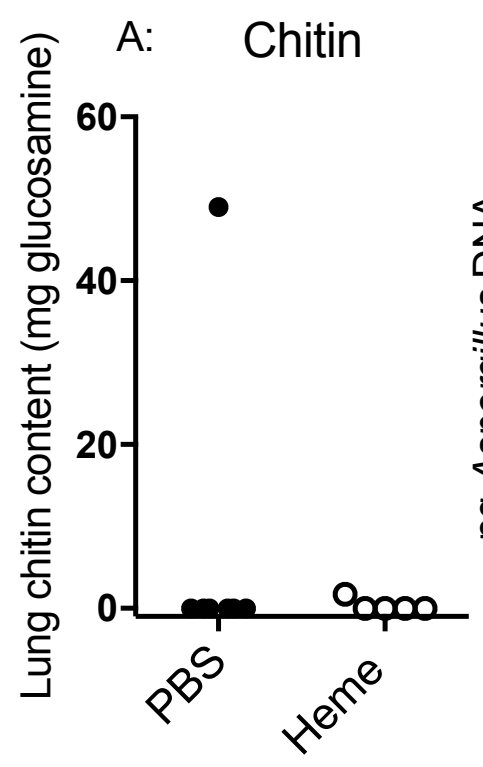

B: $q P C R$

C: Colony formation

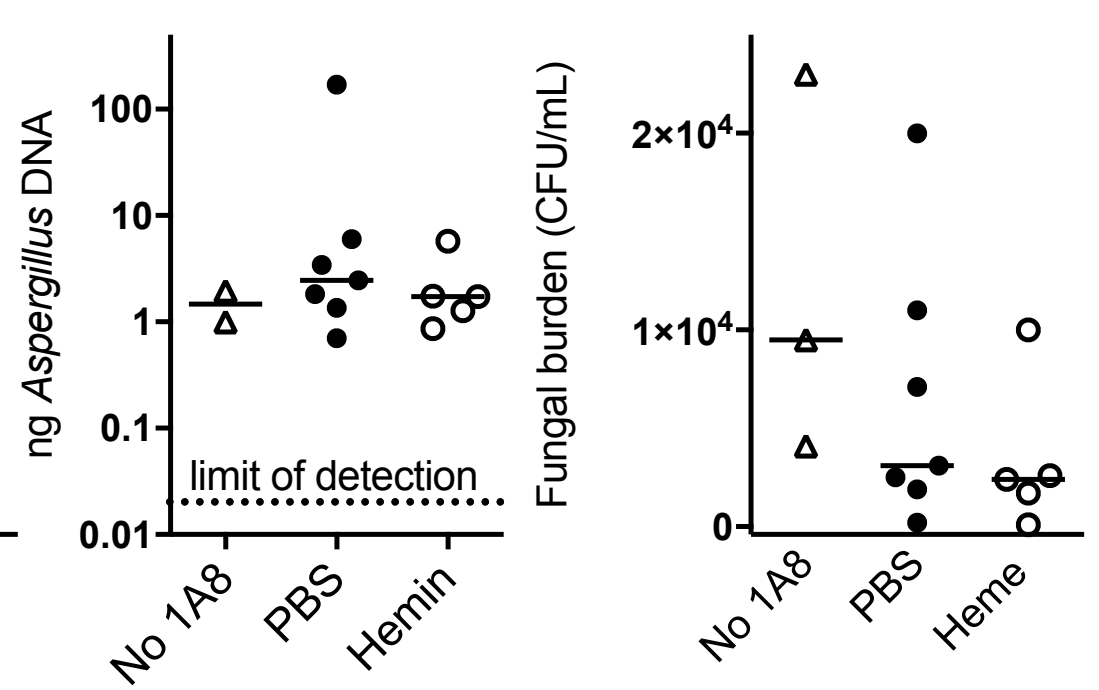


Figure 3.5. Method comparison for fungal burden assay. Neutropenic mice were treated with hemin PBS coinstilled with A. fumigatus. Three different assays were used to determine fungal burden: A: Chitin, B: A. fumigatus DNA content, and C: CFU. For B and $\mathrm{C}$, non-neutropenic mice challenged with $A$. fumigatus were used as controls to validate the method. 


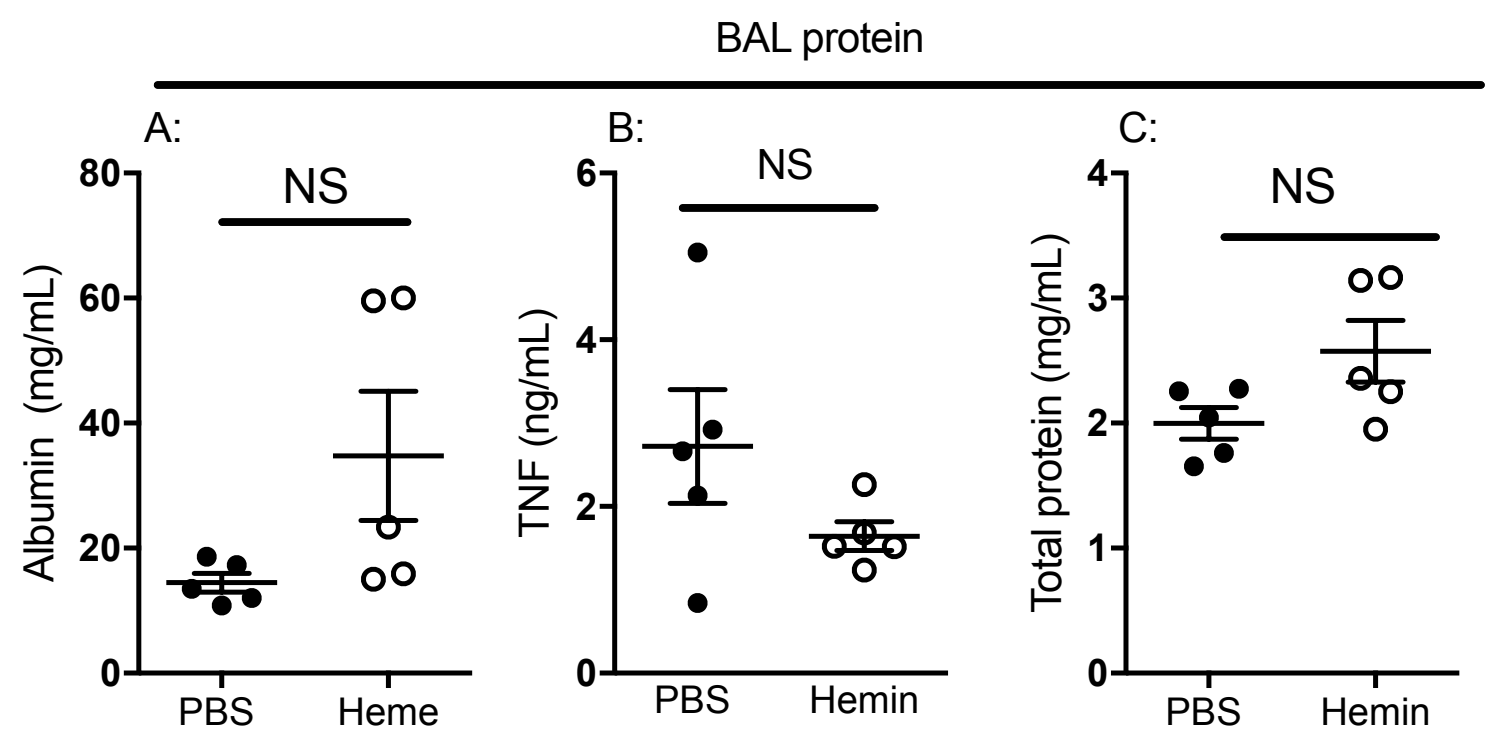


Figure 3.6. Markers of acute lung injury in mice challenged with dead $A$. fumigatus germlings. BAL fluid was recovered in a volume of $1 \mathrm{~mL}$. (A) Albumin (B) TNF (C) total protein. 


\subsection{Discussion}

In this project we investigated the role of heme release in the pathogenesis of invasive pulmonary aspergillosis. We found that, during murine invasive pulmonary aspergillosis, A. fumigatus penetrates the vasculature of the lung, induces pulmonary hemorrhage, and causes the release of heme into the extracellular space. In contrast to previous reports, we found that $A$. fumigatus was able to import and use exogenous heme for growth. Finally, we found that exogenous hemin enhances host susceptibility to invasive pulmonary aspergillosis through an as yet undefined mechanism that cannot be recapitulated by the combination of hemin and antigen alone.

Our finding that invasive pulmonary aspergillosis induced the release of cell-free heme into the alveolar space is novel. Angioinvasion is a common finding in human invasive pulmonary aspergillosis ${ }^{197,198}$. A survey of lung histology samples from invasive pulmonary aspergillosis patients found that almost all neutropenic patients and hematopoietic cell transplant recipients displayed alveolar hemorrhage, even in the absence of angioinvasion, and about half had displayed hemorrhagic infarction ${ }^{195}$. Heme release into the lung parenchyma is therefore likely to happen during human infection, as was observed experimentally in mice.

Our finding that $A$. fumigatus can utilize exogenous heme contradicts the current dogma in the field of iron uptake in fungal pathogens, but is consistent with the findings in industrial microbiology and does not directly conflict with any data that have previously been reported. Similar to the group that concluded exogenous heme is inaccessible to Aspergillus species, we found that hemin supplementation enhanced growth and conidiation in liquid media, such as that used in the industrial production of 
heterologous proteins ${ }^{120,137}$. The mechanism of heme uptake is unclear, but potentially involves passive binding to the fungal cell wall as bleach-killed hyphae bound to PPIX. It has been previously shown that $A$. fumigatus cell wall-associated proteins are composed of between 20-50 soluble proteins that are secreted from mature hyphae in stationary phase, and association with the cell wall is only incidental ${ }^{199}$. Because hemin-dependent differences in growth are apparent before stationary phase, the secreted proteins are probably not responsible for the initial utilization of heme although they may play a role at later stages in growth. Heme may bind directly to the carbohydrates in the fungal cell wall, rather than a specific protein, as heme will bind many structures (including the cellulose membranes of molecular cut-off filters). It is therefore possible that heme import at high extracellular concentrations is a passive process.

Overall, our data do not support the hypothesis that exogenous heme promotes the growth of $A$. fumigatus hyphae in lung tissue, despite worsened outcome. We observed that mice treated with A. fumigatus and hemin developed severe pneumonia, but did not have increased $A$. fumigatus penetration into lung tissue as determined by histology or higher fungal bacterial burden as determined by chitin, DNA, and CFU quantification as compared to PBS-treated mice which had mild disease. We are currently repeating these experiments using a Gr1-depleting antibody, which causes more profound immunosuppression and has permitted clear measurements of fungal burden in our hands. Interestingly, we observed that treatment with a high dose of killed A. fumigatus hyphae and hemin did not result in more severe acute lung injury or clinical score as compared to mice treated with killed $A$. fumigatus hyphae and PBS, 
suggesting that antigen alone is insufficient to drive fatal lethal inflammatory responses in this setting. At this stage, we are testing whether mice treated with hemin and dead hyphae develop severe pneumonia at later time points.

\subsection{Methods}

\subsubsection{Mice}

Male and female sex and age matched C57BL/6 mice 6-10 weeks of age were purchased from Jackson Laboratories (Bar Harbor, Maine) and maintained under standard housing conditions at the University of Virginia. Due to known interference of the microbiome with the infection model, mice were maintained for less than two weeks in the Pinn hall vivarium before use in experiments. In some experiments, mice bred at UVA were intentionally used. All protocols were approved by the Animal Care and Use Committee of the University of Virginia. Invasive pulmonary aspergillosis was induced using a previously characterized model with minor modifications ${ }^{200}$.

Briefly, we induced transient neutropenia by injecting $400 \mu \mathrm{g}$ of a depleting Ly6G monoclonal antibody (clone 1A8, BioXcell, West Lebanon, New Hampshire) one day prior to infection, resulting in 3-4 days of profound neutropenia ${ }^{200}$. A.fumigatus (strain 13073; American Type Culture Collection, Manassas, Virginia) was cultured on Sabouraud's dextrose agar (SDA) at $37^{\circ} \mathrm{C}$ for $2-3$ weeks before use in experiments. The day of infection, conidia were harvested using $0.1 \%$ Tween in PBS, filtered through sterile gauze, centrifuged at $1800 \mathrm{rpm}$, resuspended in PBS, and counted manually on a hemocytometer. Mice were anaesthetized and given $7 \times 10^{6}$ conidia via intratracheal injection in a total volume of $30 \mu \mathrm{L}$ and allowed to recover in a vertical position for 
several minutes before being returned to a heated cage. In experiments where UVA mice were used, the inoculum was adjusted to $4 \times 10^{7}$. In experiments with killed germlings, $1 \times 10^{6}$ germlings were delivered. In some experiments mice were given 100 ug of hemin (Sigma, St. Louis, Missouri), which is equivalent to heme contained in $\sim 15 \mu \mathrm{L}$ of whole blood, or a molar equivalent of Sn(IV) PPIX in PBS coinstilled with conidia. Inoculations of Sn(IV) PPIX were performed under low lighting conditions to prevent toxicity. The dose of hemin was selected empirically; in pilot studies, we determined that at a lower dose, all iron was cleared within 24 hours and that at a higher dose, mice were visibly sick from hemin administration alone.

\subsubsection{Culture of $A$. fumigatus}

Iron-free aspergillus minimal media (AMM) was prepared as previously described, omitting the addition of ferrous sulfate to Hunter's Trace elements and using a final $\mathrm{pH}$ of 6.7 instead of 6.5 , to prevent hemin from precipitating out of solution ${ }^{120}$. Hemin-supplemented or Sn(IV) protoporphyrin-supplemented media were prepared by first dissolving the powder in $1 \mathrm{~mL}$ of $0.1 \mathrm{~N} \mathrm{NaOH}$ in PBS, then diluting further to a final concentration of $500 \mu \mathrm{M}$ in minimal media. Stock solutions were autoclaved and then diluted as needed. Cultures were grown at $37^{\circ} \mathrm{C}$ in static conditions in 6 well or 24 well culture plates, depending on the assay.

To generate $A$. fumigatus germlings, conidia were harvested from SDA plates and filtered through guaze as previously described and resuspended at a concentration of $1-2 \times 10^{7}$ condia/mL in conical tubes at $80 \%$ volume to allow oxygenation. Cultures were grown overnight in a shaking incubator, and then sampled hourly in the morning to 
monitor germ tube formation. When germ tubes reached a length of $\sim 4 \mu \mathrm{M}$, the cultures were washed in PBS and then stored in $70 \%$ ethanol for 72 hours to kill hyphae and conidia. Cultures were washed extensively before murine challenge.

Dry mass was determined by removing as much fungal material as possible for liquid cultures and placing the hyphal mat conidia-side down on a piece of pre-weighed paper. Samples were allowed to air dry overnight, and the dry mass was recorded. Percent conidiation was determined by removing hyphal mats from liquid media and rinsing them for several minutes in $4 \mathrm{~mL} 2 \mathrm{mM}$ PBS $0.1 \%$ Tween, then enumerating total conidia recovered on a hemacytometer. Total mRNA was isolated by using a commercial kit (Zymogen Research, Irvine, CA) and transcribed into cDNA using a commercial kit (Promega, Madison, WI), then amplified using SYBR Green. The $A$. fumigatus-specific primer sequences used were designed using NCBI primer design and were as follows: $\beta$-actin $F \quad 5$ 'TCCAATACCTGGCGGTTTCC, $R \quad R$

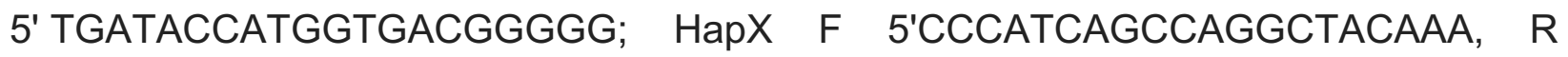
5'AGGCGTCGGCACAAGATAAA. For measurement of intracellular iron and PPIX accumulating, germlings were washed extensively in PBS $0.1 \%$ tween, then homogenized in a high-speed bead basher. Iron was measured using the ferrozine assay and PPIX fluorescence was measured at an excitation/ emission wavelength of $425 / 590 \mathrm{~nm}$.

\subsubsection{Protein, histology, and iron analysis}

Lungs were collected for histology by perfusing the vasculature with $2 \mathrm{~mL}$ cold PBS-2mM EDTA followed by $4 \%$ paraformaldehyde. The lungs were inflated with $4 \%$ 
paraformaldehyde under a constant pressure of $30 \mathrm{~cm}$ water, fixed in $4 \%$ paraformaldehyde overnight, then stored in $70 \%$ ethanol. Fixed lungs were embedded in paraffin and sequential slides were taken for histology. Fungal structures in the lung were identified using a modified Grocott methanamine silver stain (Sigma Aldrich, St.Louis MO). BAL was harvested by cannulating the trachea, inflating the lungs with 1 $\mathrm{mL}$ of PBS-2mM EDTA, and recovering the fluid. Iron in the BAL was determined using the ferrozine assay ${ }^{201}$. Albumin, total protein, and TNF were measured using commercial kits (Abcam, Cambridge, UK; Bio-Rad, Hercules, CA; and R\&D Biosystems, Minneapolis, MN respectively). Hemoglobin in the whole lung was measured using a commercial kit (Cayman Chemical, Ann Arbor, MI).

Chitin was measured using the chitin assay ${ }^{202}$. CFU was determined by plating lung homogenates on SD plates. A. fumigatus DNA content was determined by extracting DNA from the homogenized lungs of infected mice using a commercial kit (Zymogen Research, Irvine, California). A standard curve prepared from A. fumigatus conidia was used to estimate lung DNA content. 


\section{Chapter IV: Concluding remarks and future directions}

\subsection{Summary}

This thesis dissertation contributes to our knowledge of the complex interplay between iron metabolism and pneumonia. In Chapter II, we demonstrated using a murine model of Klebsiella pneumonia that even transient elevation in plasma iron permits bacterial outgrowth in the bloodstream, resulting in disseminated and rapidly fatal disease (Figure 4.1). Furthermore, we demonstrated that hepcidin mimetics are able to rescue iron-overloaded mice from infection. This research contributes to the field of iron during infection because we are the first to show that hepcidin mimetics are protective against pneumonia, and the first to show hepcidin-mediated iron sequestration is a critical defense mechanism against a very common pathogen, rather than one which selectively affects iron-overloaded individuals.

These data provide the host counterpart to the existing literature on the role of

bacterial siderophore system in the virulence of Gram-negative pathogens ${ }^{96,203}$. The hypervirulent strain of $K$. pneumoniae used in this study produces yersiniabactin, which supports bacterial growth in the respiratory tract, and glycosylated enterobactin, which promotes bacterial growth in the bloodstream by evading lipocalin-2, a host-derived molecule that sequesters non-glycosylated enterobactin and limits microbial iron uptake $82,97-99,103$. Our findings indicate that even bacteria equipped with these high affinity acquisition systems are susceptible to a host mechanism that limits iron availability in the host environment. As such, we speculate that these findings may be broadly applicable to infections caused by other phylogenetically related aerobic Gramnegative pathogens ${ }^{204}$. 
This work has several implications for future research. First, our results may have implications for the therapeutic use of hepcidin antagonists, which are currently under development for the treatment of anemia of inflammation. In this context, we posit that relieving the iron-restricted state could predispose some individuals to Gram-negative sepsis. Second, impaired hepcidin production is a feature of several common clinical entities, including alcohol intoxication and chronic liver disease ${ }^{156-159}$, illnesses associated with striking susceptibility to Gram-negative bacteremia ${ }^{205,206}$. Our data suggest that impaired hepcidin production may represent an unappreciated mechanism predisposing these hosts to infection. Finally, hepcidin agonists may represent a novel therapeutic strategy in illnesses where hepcidin production is diminished, such as liver disease and hereditary hemochromatosis, and possibly more broadly in acquired ironoverload states. Hepcidin mimetics are currently in Phase I Clinical trials for use in iron overload disorders, and our research validates that this therapy has an additional lifesaving application in the context of infection and pneumonia. 
A:

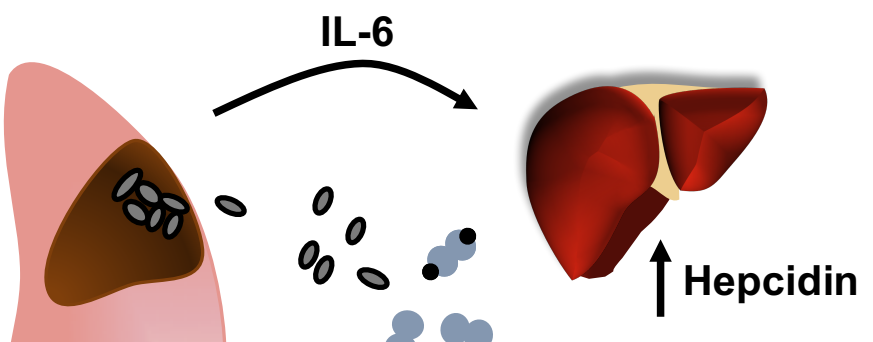

B:
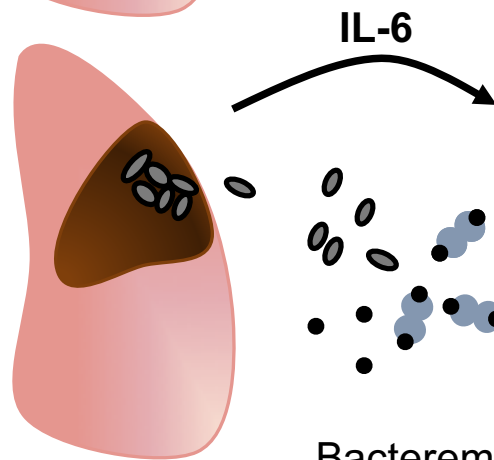

0

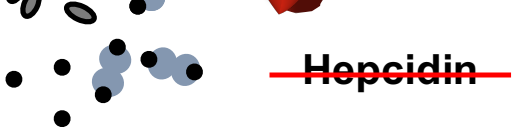

Bacteremia

C:

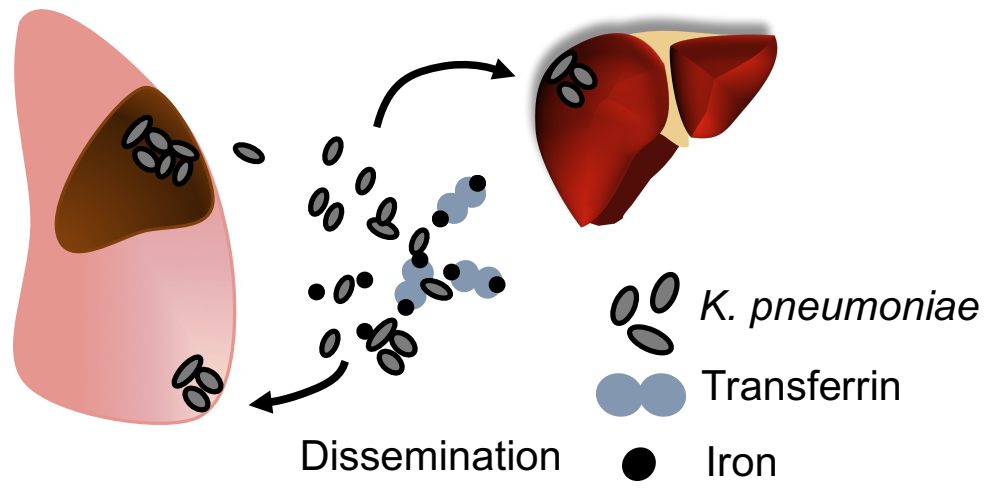


Figure 4.1. Proposed role of hepcidin during Gram-negative pneumonia. A: During pneumonia in a normal host, hepcidin is induced in the liver that causes intracellular iron sequestration and subsequent hypoferremia. Bacteria seeding the bloodstream from the lung grow poorly. B: In a hepcidin-deficient host, plasma iron increases and leads to the appearance of non-transferrin bound iron. Bacteria seeding the bloodstream from the lung are able to grow. C: In an iron-rich setting during hepcidin deficiency, bacteria grow in the bloodstream and disseminate to other infection sites, causing more severe disease and death. 


\subsection{Is hepcidin dysregulation a component of cirrhosis-mediated susceptibility to infection?}

\subsubsection{Preliminary data}

While we used a model of genetic hepcidin deficiency to study the effects of iron sequestration in host defense against pneumonia, poor hepcidin production has been reported in patients with liver cirrhosis and fibrosis of diverse etiologies. Induction of experimental liver cirrhosis sensitizes mice to infection during controlled experiments ${ }^{207-}$ 210 . Interestingly, many of the patient groups with poor hepcidin production are also very susceptible to Gram-negative bacteremic infections. The most striking example is alcoholic cirrhosis. Alcoholics are far more likely to present with Klebsiella pneumonia compared to the general population, and are more likely to die from infection ${ }^{211}$. Notably, alcoholics are susceptible to severe, invasive bacteremic $K$. pneumoniae infections with exceptionally poor outcomes ${ }^{206}$. The mechanism behind susceptibility to pneumonia in alcoholics is multi-faceted and involves impaired complement production, increased risk of aspirating bacteria from the gastrointestinal tract, and disrupted barrier function of the lung; however, the basis of susceptibility is incompletely understood

In human patients, alcoholic cirrhosis is associated with progressive iron overload, and low serum hepcidin levels are associated with poor outcomes ${ }^{157,212}$. Experimental studies in mice and rats suggest that alcohol consumption inhibits the ability of hepatocytes to produce hepcidin in response to dietary iron ${ }^{158,212}$. While the mechanism of hepcidin downregulation is not clear, both groups suggest alcohol mediated oxidative damage to hepatocytes reduces $\mathrm{C} / \mathrm{EBP} \alpha$ expression, potentially disrupting hepcidin 
transcription. HCV infection similarly blunts hepcidin induction in response to iron, and is associated with progressive iron overload ${ }^{156}$.

Because our data strongly suggest that hepcidin-mediated iron regulation is critical for host defense against $K$. pneumoniae, we hypothesized that hepcidin dysregulation contributes to susceptibility to invasive bacterial infections in cirrhosis patients (Figure 4.2A). In order to determine if experimentally induced liver fibrosis altered hepcidin expression in mice, we analyzed samples that were collected several years ago to address an unrelated hypothesis. Mice were fed chow containing 3,5-diethoxycarbonyl1,4-dihydrocollidine (DDC) or a control diet for 4 weeks to induce liver fibrosis. We found that mice fed DDC had elevated plasma iron levels as compared to mice fed the control diet (Figure 4.2B), suggesting that DDC treatment disrupted iron metabolism. We found that mice fed DDC had elevated hepatic iron stores compared to control-fed mice after 4 weeks (Figure 4.2C), once again suggesting DDC treatment altered iron metabolism. Interestingly, mice that were fed DDC chow did not have elevated hepcidin expression despite the presence of high plasma iron levels, suggesting that hepcidin upregulation in response to high iron levels is inhibited during liver fibrosis (Figure 4.2D). An area of future study would be to test whether mice with experimental liver disease are more susceptible to Klebsiella pneumonia, and if liver fibrosis interferes with hepcidin-mediated iron sequestration during infection. 
A:
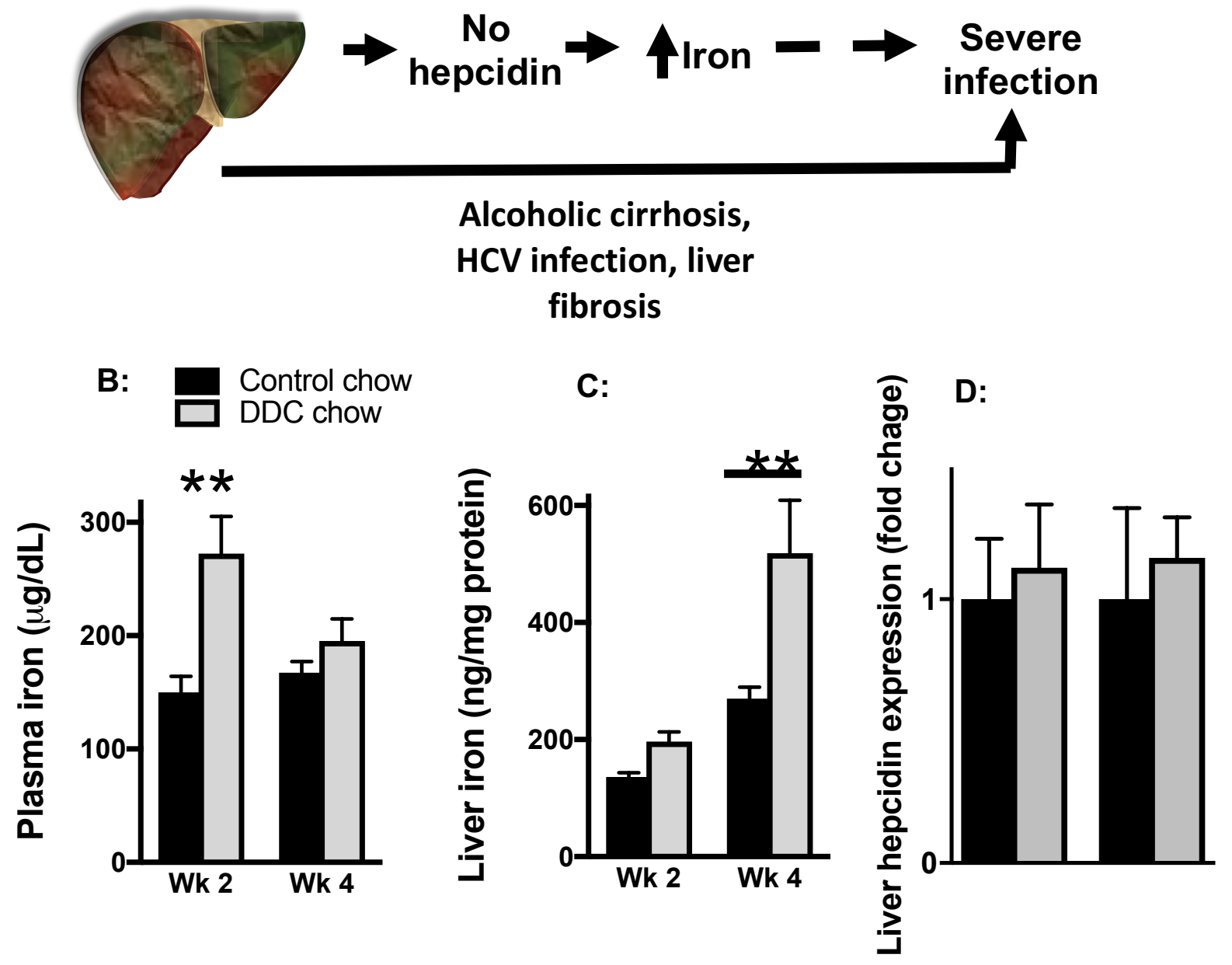
Figure 4.2. Does hepcidin dysregulation contribute to infection during cirrhosis? A: Model summarizing the potential role of hepcidin dysregulation in the susceptibility of cirrhosis patients to infectious disease. Bold arrows represent pathways that have been shown to be true, dashed arrow represents a hypothetical pathway. Mice were fed a diet containing DDC to induce liver fibrosis, or a control diet, for the indicated timespan. Iron levels present in the B: plasma and C: liver following DDC treatment. D: Liver hepcidin mRNA. $N=5$ per group ${ }^{* *} p<0.01$ one way ANOVA Tukey post-test. 


\subsection{2: Future experiments}

We propose to test the therapeutic potential of mini hepcidins in liver disease by using a murine liver-fibrosis and pneumonia model. In order to show proof-of-principle that mini-hepcidins can have a therapeutic role in human illnesses where hepcidin production is impaired, we could assess their use in mice with carbon tetrachloride $\left(\mathrm{CCl}_{4}\right)$-induced liver fibrosis. While our preliminary experiments were conducted using mice maintained on DDC, we have determined that $\mathrm{CCl}_{4}$ is a more appropriate liver fibrosis model for our application.

Four cohorts of mice would be maintained on $\mathrm{CCl}_{4}$ or control chow for 6 weeks before intratracheal challenged with $K$. pneumoniae, and then treated during infection with either vehicle or mini hepcidin (Table 4.1). Our hypothesis predicts that, as compared to control mice, animals treated with $\mathrm{CCl}_{4}$ will have blunted hepcidin production, increased plasma LPI associated with more severe infection, and that treatment with mini hepcidin will result in reduced plasma LPI and better infection outcome. Given the lack of hepcidin induction in the DDC model of chronic liver injury in Figure 4.1, we anticipate that this model will demonstrate impaired hepcidin production. Another possible approach would be to use chronic ethanol consumption to induce hepcidin suppression, which has been validated in mice and is a robust counterpart to human disease. In further follow-up experiments, we would treat four cohorts of mice with $\mathrm{CCl}_{4}$ for 6 weeks, induce pneumonia using clinical K. pneumoniae isolates, and treat mice with vehicle only, antibiotics, mini hepcidin, or both. In this experiment, we would test the hypothesis that mini hepcidin administration will have an additive benefit over antibiotics alone in clinically relevant species (Table 4.2). If the mini-hepcidin, 
proves effective in our mouse pneumonia models, these experiments will provide rationale for the development of hepcidin mimetics as novel treatments in infections with Gram-negative organisms in susceptible hosts. 


\begin{tabular}{|c|c|c|c|c|}
\hline Group & \multicolumn{2}{|c|}{ Mouse } & Treatments & Measurements \\
\hline 1 & WT & $\mathrm{CCl}_{4}$ & Vehicle & $\begin{array}{l}\text { - Lung and Bacterial Burden } \\
\text { on Day } 2\end{array}$ \\
\hline 2 & WT & $\mathrm{CCl}_{4}$ & Mini hepcidin & $\begin{array}{l}\text { - Plasma and BAL transferrin } \\
\text { iron and LPI }\end{array}$ \\
\hline 3 & WT & Vehicle & Vehicle & - Liver hepcidin transcript; \\
\hline 4 & WT & Vehicle & Mini hepcidin & plasma hepcidin protein \\
\hline
\end{tabular}


Table 4.1. Experimental groups to test the role of dysregulated iron metabolism during liver fibrosis. These proposed experimental groups will test the hypothesis that hepcidin-mediated iron sequestration is inhibited during liver fibrosis, and investigate the utility of hepcidin mimetics in the context of pneumonia and liver fibrosis. 


\begin{tabular}{|c|c|c|c|c|c|}
\hline Group & Mou & & Treatments & Day 0 & Measurements \\
\hline 1 & WT & $\mathrm{CCl}_{4}$ & Vehicle alone & \multirow{4}{*}{$\begin{array}{l}\text { Intratracheal } \\
\text { clinical } \\
\text { bacterial } \\
\text { isolates }\end{array}$} & \multirow{4}{*}{$\begin{array}{l}\text { - Lung and Bacterial Burden } \\
\text { on Day } 2 \\
\text { - Plasma and BAL transferrin } \\
\text { iron and LPI } \\
\text { - Liver hepcidin transcript; } \\
\text { plasma hepcidin protein }\end{array}$} \\
\hline 2 & WT & $\mathrm{CCl}_{4}$ & Antibiotics & & \\
\hline 3 & WT & $\mathrm{CCl}_{4}$ & Mini hepcidin & & \\
\hline 4 & WT & $\mathrm{CCl}_{4}$ & $\begin{array}{l}\text { Antibiotics + } \\
\text { minihepcidin }\end{array}$ & & \\
\hline
\end{tabular}


Table 4.2: Experimental groups to test the hypothesis that hepcidin mimetics can be used against clinical isolates. This experiment will test the hypothesis that hepcidin mimetics can enhance the treatment of Gram-negative pneumonia and bacteremia caused by clinic isolates in hosts with liver fibrosis. 


\subsection{Do infections stimulate a release of iron from cells?}

\subsubsection{Preliminary data}

Although hepcidin-independent mechanisms of hypoferremia have been reported after challenge with inflammatory stimuli ${ }^{23,149,151}$, we found that hypoferremia during pneumonia was completely abrogated in hepcidin-deficient mice, and in fact, plasma iron levels became elevated following infection in hepcidin-deficient mice (reproduced in Figure $4.3 \mathrm{~A}$ for comparison). Our use of an infectious agent, rather than sterile inflammatory stimuli used in other reports, may account for this difference. We speculate that this increase in plasma iron levels reflects decreases in iron consumption by cytokine-suppressed erythropoiesis ${ }^{150}$, and release of iron from intracellular sources damaged by infection, such as the liver ${ }^{213}$. We thus propose a model in which infections result in a tendency towards increased extracellular iron that benefits the pathogen, and the protective role of inflammation-mediated hepcidin induction is to counteract this phenomenon. We speculate that the excess iron originates from damaged hepatocytes, although hepcidin-/- mice do not have worse liver damage following infection than WT mice. Interestingly, we found that hepcidin-/- mice also developed an increase in plasma iron levels following challenge with invasive pulmonary aspergillosis (Figure 4.3B), suggesting that hyperferremia in the context of hepcidin deficiency during infection is not unique to Gram-negative infections. We also observed that WT mice treated with a pharmacological hepcidin inhibitor developed increased plasma iron in response to infection compared to mice treated with vehicle alone (Figure 4.3C). In this setting, we suggest that the excess iron is a result of red 
blood cells lysing during pulmonary hemorrhage. Taken together, these results suggest that iron is somehow released into the plasma during certain infections.

There are several lines of evidence that iron can leak from damaged cells into the bloodstream. Serum ferritin is increased both during iron overload, and independently, during inflammation. There is debate over whether ferritin is actively secreted into the bloodstream in response to inflammation and iron overloading, or if serum ferritin is debris from damaged cells ${ }^{213,214}$. It appears that in some circumstances, serum ferritin is a leakage product. A study looking at a cohort of nonalcoholic fatty liver disease patients found that elevated serum ferritin strongly predicted severe liver fibrosis histology scores, but had no relationship to iron overload, suggesting that liver damage causes release of ferritin independently from iron stores ${ }^{215}$. In a cohort of humans with sickle cell disease, serum ferritin and hepatic iron overload were strongly correlated up to moderate-high levels, but very high levels of serum ferritin predicted liver fibrosis rather than very high iron stores ${ }^{216}$. Another study centered around liver cirrhosis patients found that hepatocellular damage strongly correlated with serum ferritin that had a high iron content unrelated to hepatic iron stores, suggesting that during liver damage holo-ferritin is released from cells ${ }^{217}$.

Based on correlative research in human patients and observations in our lab, we hypothesize that hepcidin counters the release of iron from damaged cells during infection. The benefit of hepcidin induction during infection may be the prevention of hyperferremia, particularly the appearance of LPI, rather than the induction of hypoferremia. This mechanism may be particularly important in the defense against pathogens which infect hepatocytes or induce hemolysins. 


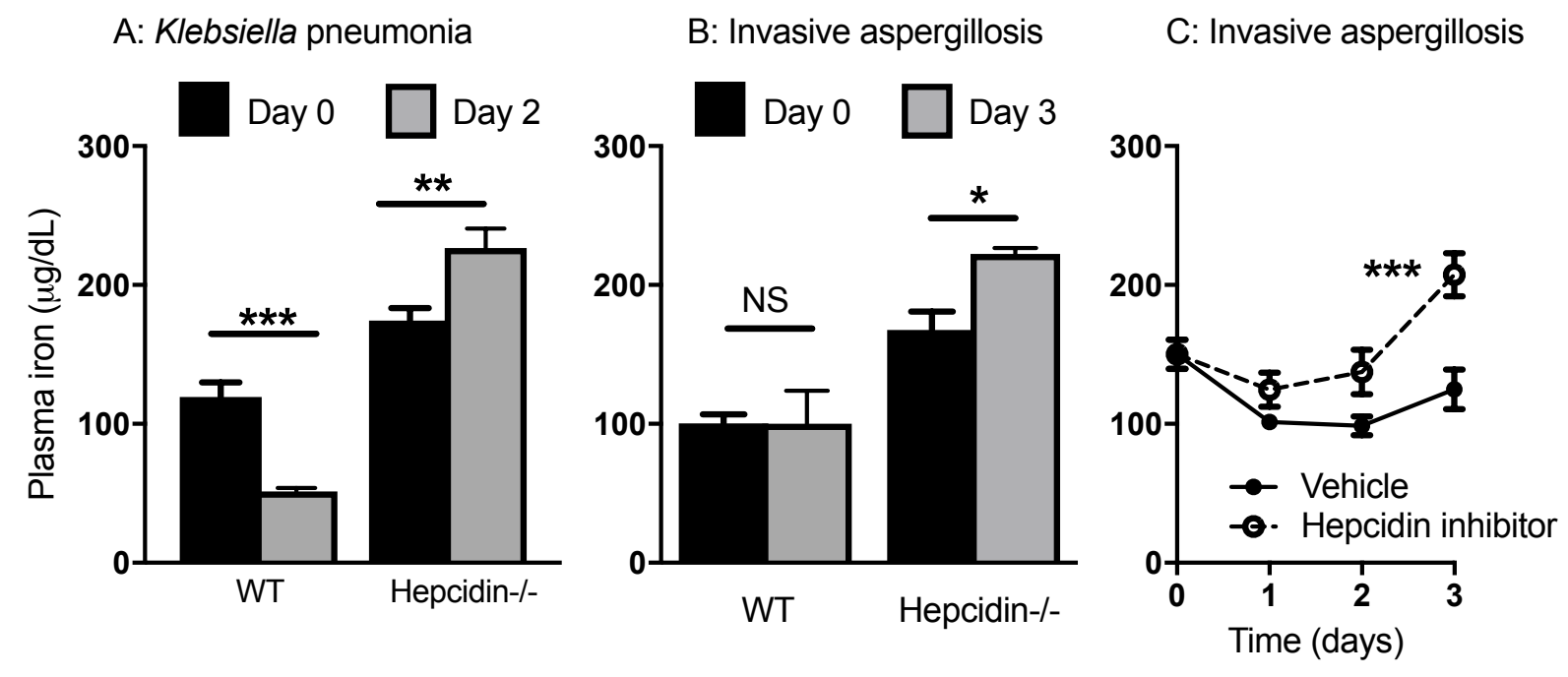


Figure 4.3: Evidence of hyperferremia following infection during hepcidin deficiency. A) Plasma iron levels of hepcidin-/- and WT mice at baseline and 2 days after induction of Klebsiella pneumonia. Modified from Figure 2.4A to emphasize comparison. $\mathrm{N}=13-15$ per group, combined result of 3 independent experiments; ${ }^{* *}$ and *** denote $P$ values of 0.01 and 0.001 respectively, one-way ANOVA post-test analysis. B) Plasma iron levels of neutropenic hepcidin-/- and WT mice at baseline and 3 days following induction of invasive pulmonary aspergillosis. $N=3-5$ per group; ${ }^{* *}$ and ${ }^{* * *}$ denote $P$ values of 0.01 and 0.001 respectively, one way ANOVA post-test analysis. C) Plasma iron values in neutropenic mice given the BMPR inhibitor Ldn-193189 (hepcidin inhibitor) or vehicle control $3 \times$ daily following induction of invasive pulmonary aspergillosis. $\mathrm{N}=5-9$ per group, ${ }^{* * *}$ denote $P$ value of 0.001 , two-way ANOVA. 


\subsubsection{Future experiments}

A possible approach to determine if infections stimulate the release of iron from damaged cells is to use pulse-chase experiments using ${ }^{59} \mathrm{Fe}$. Prior to infection, WT and hepcidin-/- mice would be given a dose of unradiolabeled iron to saturate circulating transferrin levels, followed by a dose of radiolabeled iron. It has been previously shown that radiolabeled iron is imported by the liver under these circumstances ${ }^{4}$. After several treatments of radiolabeled iron, the mice would be infected with $K$. pneumoniae and monitored for several days. If infection does release iron from intracellular stores, we would expect to see a burst of radioactive iron in the plasma of both WT and hepcidin-/mice on day 2 as compared to uninfected animals. Although both hepcidin-/- and wildtype mice received the same amount of radioactive iron, we would expect to see higher levels of radioactive iron in the plasma of hepcidin-/- mice as compared to wildtype mice due to failed clearance from the blood following release. We would expect the radioactive iron to accumulate in the spleen of wildtype mice, but not hepcidin-/- mice, as the knockout mice have reduced capacity to sequester the released iron.

A similar approach could be used to test the hypothesis that hepcidin induction iron release from in vivo hemolysis. Several weeks prior to infection, hepcidin-/- and WT mice would be given non-transferrin-saturating doses of ${ }^{59} \mathrm{Fe}$ in repeated intervals. Based on prior radiolabeling studies, the majority $(80 \%)$ of injected iron would be incorporated into erythrocytes within 48 hours $^{218}$. Several days after the iron treatment, mice would be neutrophil depleted and then infected the following day with $A$. fumigatus. If iron from red blood cells is released during infection, we would expect a 
release of radioactive iron in the plasma during infection in both WT and hepcidin-/mice. We would expect the released iron to accumulate in the spleen of WT, but not hepcidin-/-, mice.

\subsection{Future directions in the study of $A$. fumigatus and heme uptake}

In Chapter III, we challenged the standing dogma in the field of medical mycology that Aspergillus species are unable to use host-derived heme as an iron scource. We demonstrated that $A$. fumigatus is able to use exogenous hemin in vitro, and present evidence that $A$. fumigatus comes in direct contact with erythrocytes in vivo during murine infection. This finding opens up a new line of research to investigate the role of heme acquisition in invasive pulmonary aspergillosis, and the potential to target heme uptake as a treatment pathway.

Currently, our data suggest that exogenous hemin promotes more severe disease that is independent on hyphal growth, but that cannot be recapitulated with killed hyphal forms alone. There are several possible explanations for this phenomenon. First, it is possible that live hyphae shed antigens or PAMPS, such as galactommanan, that are not shed by killed hyphae that are responsible for driving pathogenic immune responses. Second, it is possible that exogenous heme does not produce enhanced hyphal growth, but does enhance the production of enzymes that damage and degrade the lung parenchyma. The ability of heme to enhance the pathogenesis of invasive pulmonary aspergillosis may thus be important in human infections as either a mechanism severe immunopathology, or of as an agent of enhanced virulence without a change in growth. 
We have identified several candidate genes which may be differentially regulated during infection to enhance virulence. Cat1 and cat2 encode hyphal catalases, which are heme-containing enzymes. Catalase neutralizes reactive oxygen species and promote disease establishment during invasive pulmonary aspergillosis. The cat 1 and cat2 products are secreted by $A$. fumigatus in culture and are recognized by antibodies of the sera of invasive pulmonary aspergillosis patients ${ }^{219,220}$. GliZ and gliP gene products mediate the production of gliotoxin, a secondary metabolite that has immunosuppressive effects ${ }^{221}$. While there is not an obvious connection between heme metabolism and gliotoxin production, gliotoxin is regardless one of the few true virulence factors of $A$. fumigatus and should be studied in this context ${ }^{222}$. Finally, we are also investigating the regulation of 3 fatty acid oxygenases, which are heme containing enzymes, that have been shown to modify host arachidonic acid acids and produce species such as prostaglandins that can dramatically affect immune recruitment ${ }^{223}$.

Currently, we are also working to visualize uptake of PPIX by A. fumigatus in the lungs of infected mice to further interrogate whether heme uptake occurs during infection. While we can successfully visualize PPIX dispersal throughout the lung using confocal microscopy with little endogenous background, our preliminary findings indicate that alveolar epithelial cells and macrophages both uptake administered PPIX, and that hyphal PPIX uptake is not readily visualized against the staining of other tissues. We are currently working to combine direct PPIX uptake with immunofluorescence to determine whether exogenous PPIX is imported by hyphae and conidia. 
While we have shown that exogenous heme does not appear to promote hyphal growth, a separate question is whether the endogenous release of heme or hemoglobin enhance infection. The most direct way to address this would be to identify, target, and disrupt a putative $A$. fumigatus heme transporter and study the effect on fungal virulence during murine infection. As previously discussed, it is not entirely clear that heme uptake is driven by a dedicated receptor or protein in A. fumigatus. Currently, we are working to develop a method to visualize heme uptake in vivo using PPIX. Another strategy is to neutralize the haptoglobin and hemopexin-mediated clearance of hemoglobin and heme. There are currently no commercially available monoclonal antibodies against either protein or cognate receptor that have been validated for in vivo use in mice, but both haptoglobin and hemopexin-deficient mice have been generated already and may prove useful in future studies. 


\section{References}

1. Hentze, M. W., Muckenthaler, M. U. \& Andrews, N. C. Balancing Acts: Molecular Control of Mammalian Iron Metabolism. Cell 117, 285-297 (2004).

2. Skaar, E. P. The battle for iron between bacterial pathogens and their vertebrate hosts. PLoS Pathog. 6, e1000949 (2013).

3. Gosriwatana, I. et al. Quantification of non-transferrin-bound iron in the presence of unsaturated transferrin. Anal. Biochem. 273, 212-220 (1999).

4. Craven, C. M. et al. Tissue distribution and clearance kinetics of non-transferrinbound iron in the hypotransferrinemic mouse: a rodent model for hemochromatosis. Proc. Natl. Acad. Sci. 84, 3457-3461 (1987).

5. Theil, E. C. Ferritin: structure, gene regulation, and cellular function in animals, plants, and microorganisms. Annu. Rev. Biochem. 56, 289-315 (1987).

6. Nicolas, G. et al. Lack of hepcidin gene expression and severe tissue iron overload in upstream stimulatory factor 2 (USF2) knockout mice. Proc. Natl. Acad. Sci. 98, 8780-8785 (2001).

7. Park, C. H., Valore, E. V., Waring, A. J. \& Ganz, T. Hepcidin, a urinary antimicrobial peptide synthesized in the liver. J. Biol. Chem. 276, 7806-7810 (2001).

8. Pigeon, C. et al. A new mouse liver-specific gene, encoding a protein homologous to human antimicrobial peptide hepcidin, is overexpressed during iron overload. $\mathrm{J}$. Biol. Chem. 276, 7811-7819 (2001).

9. Nemeth, E. et al. Hepcidin, a putative mediator of anemia of inflammation, is a type II acute-phase protein. Blood 101, 2461-2463 (2003). 
10. Nemeth, E. et al. Hepcidin regulates cellular iron efflux by binding to ferroportin and inducing its internalization. Science 306, 2090-2093 (2004).

11. Khan, F. A., Fisher, M. A. \& Khakoo, R. A. Association of hemochromatosis with infectious diseases: expanding spectrum. Int. J. Infect. Dis. 11, 482-487 (2007).

12. Zumerle, S. et al. Targeted disruption of hepcidin in the liver recapitulates the hemochromatotic phenotype. Blood 123, 3646-3650 (2014).

13. Nicolas, G. et al. The gene encoding the iron regulatory peptide hepcidin is regulated by anemia, hypoxia, and inflammation. J. Clin. Invest. 110, 1037-1044 (2002).

14. Kautz, L. et al. Identification of erythroferrone as an erythroid regulator of iron metabolism. Nat. Genet. 46, 678 (2014).

15. Babitt, J. L. et al. Bone morphogenetic protein signaling by hemojuvelin regulates hepcidin expression. Nat. Genet. 38, 531-539 (2006).

16. Andriopoulos Jr, B. et al. BMP6 is a key endogenous regulator of hepcidin expression and iron metabolism. Nat. Genet. 41, 482-487 (2009).

17. Wrighting, D. M. \& Andrews, N. C. Interleukin-6 induces hepcidin expression through STAT3. Blood 108, 3204-3209 (2006).

18. Casanovas, G., Mleczko-Sanecka, K., Altamura, S., Hentze, M. W. \& Muckenthaler, M. U. Bone morphogenetic protein (BMP)-responsive elements located in the proximal and distal hepcidin promoter are critical for its response to HJV/BMP/SMAD. J. Mol. Med. 87, 471-480 (2009). 
19. Canali, S. et al. Activin B induces noncanonical SMAD1/5/8 signaling via BMP type I receptors in hepatocytes: evidence for a role in hepcidin induction by inflammation in male mice. Endocrinology 157, 1146-1162 (2016).

20. Rodriguez, R. et al. Hepcidin induction by pathogens and pathogen-derived molecules is strongly dependent on interleukin-6. Infect. Immun. 82, 745-752 (2014).

21. Leal Jr, S. M. et al. Targeting iron acquisition blocks infection with the fungal pathogens Aspergillus fumigatus and Fusarium oxysporum. PLoS Pathog. 9, e1003436 (2013).

22. Nemeth, E. et al. IL-6 mediates hypoferremia of inflammation by inducing the synthesis of the iron regulatory hormone hepcidin. J. Clin. Invest. 113, 1271-1276 (2004).

23. Kim, A. et al. A mouse model of anemia of inflammation: complex pathogenesis with partial dependence on hepcidin. Blood 123, 1129-1136 (2014).

24. Peyssonnaux, C. et al. TLR4-dependent hepcidin expression by myeloid cells in response to bacterial pathogens. Blood 107, 3727-3732 (2006).

25. Armitage, A. E. et al. Hepcidin regulations by innate immune and infectious stimuli. Blood 118, 4129-4139 (2011).

26. Nguyen, N.-B., Callaghan, K. D., Ghio, A. J., Haile, D. J. \& Yang, F. Hepcidin expression and iron transport in alveolar macrophages. Am. J. Physiol. - Lung Cell. Mol. Physiol. 291, L417-L425 (2006).

27. Zhang, X. \& Rovin, B. H. Beyond anemia: hepcidin, monocytes and inflammation. Biol. Chem. 394, 231-238 (2013). 
28. Sow, F. B. et al. Expression and localization of hepcidin in macrophages: a role in host defense against tuberculosis. J. Leukoc. Biol. 82, 934-945 (2007).

29. Enns, C. A. et al. Increased iron loading induces Bmp6 expression in the nonparenchymal cells of the liver independent of the BMP-signaling pathway. PloS One 8, e60534 (2013).

30. Core, A. B., Canali, S. \& Babitt, J. L. Hemojuvelin and bone morphogenetic protein (BMP) signaling in iron homeostasis. Front. Pharmacol. 5, 104 (2014).

31. Ramey, G., Deschemin, J.-C. \& Vaulont, S. Cross-talk between the mitogen activated protein kinase and bone morphogenetic protein/hemojuvelin pathways is required for the induction of hepcidin by holotransferrin in primary mouse hepatocytes. Haematologica 94, 765-772 (2009).

32. Truksa, J., Peng, H., Lee, P. \& Beutler, E. Bone morphogenetic proteins 2 , 4, and 9 stimulate murine hepcidin 1 expression independently of $\mathrm{Hfe}$, transferrin receptor 2 (Tfr2), and IL-6. Proc. Natl. Acad. Sci. 103, 10289-10293 (2006).

33. Silvestri, L. et al. The Serine Protease Matriptase-2 (TMPRSS6) Inhibits Hepcidin Activation by Cleaving Membrane Hemojuvelin. Cell Metab. 8, 502-511 (2008).

34. Latour, C. et al. Erythroferrone contributes to hepcidin repression in a mouse model of malarial anemia. Haematologica 102, 60-68 (2017).

35. Distante, S. et al. The origin and spread of the HFE-C282Y haemochromatosis mutation. Hum. Genet. 115, 269-279 (2004).

36. Weinberg, E. D. Survival advantage of the hemochromatosis C282Y mutation. Perspect. Biol. Med. 51, 98-102 (2008). 
37. Chlosta, S. et al. The iron efflux protein ferroportin regulates the intracellular growth of Salmonella enterica. Infect. Immun. 74, 3065-3067 (2006).

38. Nairz, M. et al. Absence of functional Hfe protects mice from invasive Salmonella enterica serovar Typhimurium infection via induction of lipocalin-2. Blood 114, 3642-3651 (2009).

39. Chen, Q. et al. Silencing airway epithelial cell-derived hepcidin exacerbates sepsisinduced acute lung injury. Crit. Care 18, 470 (2014).

40. Paradkar, P. N. et al. Iron depletion limits intracellular bacterial growth in macrophages. Blood 112, 866-874 (2008).

41. Kim, D.-K. et al. Inverse agonist of estrogen-related receptor y controls Salmonella typhimurium infection by modulating host iron homeostasis. Nat. Med. 20, 419-424 (2014).

42. Yuki, K. E. et al. Suppression of hepcidin expression and iron overload mediate Salmonella susceptibility in Ankyrin 1 ENU-induced mutant. PloS One 8, e55331 (2013).

43. Wang, L. et al. Attenuated inflammatory responses in hemochromatosis reveal a role for iron in the regulation of macrophage cytokine translation. J. Immunol. 181, 2723-2731 (2008).

44. Piperno, A. et al. Blunted hepcidin response to oral iron challenge in HFE-related hemochromatosis. Blood 110, 4096-4100 (2007).

45. Frazer, D. M. et al. Increased hepcidin expression and hypoferraemia associated with an acute phase response are not affected by inactivation of HFE. Br. J. Haematol. 126, 434-436 (2004). 
46. Constante, M. et al. Distinct requirements for Hfe in basal and induced hepcidin levels in iron overload and inflammation. Am. J. Physiol. Gastrointest. Liver Physiol. 291, G229-G237 (2006).

47. Boelaert, J. R., Vandecasteele, S. J., Appelberg, R. \& Gordeuk, V. R. The Effect of the Host's Iron Status on Tuberculosis. J. Infect. Dis. 195, 1745-1753 (2007).

48. Gordeuk, V. R., McLaren, C. E., MacPhail, A. P., Deichsel, G. \& Bothwell, T. H. Associations of iron overload in Africa with hepatocellular carcinoma and tuberculosis: Strachan's 1929 thesis revisited. Blood 87, 3470-3476 (1996).

49. Johnson, E. E., Sandgren, A., Cherayil, B. J., Murray, M. \& Wessling-Resnick, M. Role of Ferroportin in Macrophage-Mediated Immunity. Infect. Immun. 78, 5099$5106(2010)$.

50. Olakanmi, O., Schlesinger, L. S. \& Britigan, B. E. Hereditary hemochromatosis results in decreased iron acquisition and growth by Mycobacterium tuberculosis within human macrophages. J. Leukoc. Biol. 81, 195-204 (2007).

51. Olakanmi, O., Schlesinger, L. S., Ahmed, A. \& Britigan, B. E. Intraphagosomal Mycobacterium tuberculosis Acquires Iron from Both Extracellular Transferrin and Intracellular Iron Pools: IMPACT OF INTERFERON- I $^{3}$ AND HEMOCHROMATOSIS. J. Biol. Chem. 277, 49727-49734 (2002).

52. Gomes-Pereira, S., Rodrigues, P. N., Appelberg, R. \& Gomes, M. S. Increased Susceptibility to Mycobacterium avium in Hemochromatosis Protein HFE-Deficient Mice. Infect. Immun. 76, 4713-4719 (2008).

53. Ben-Othman, R. et al. Leishmania-Mediated Inhibition of Iron Export Promotes Parasite Replication in Macrophages. PLoS Pathog 10, e1003901 (2014). 
54. Omara, F. O. \& Blakley, B. R. The effects of iron deficiency and iron overload on cell-mediated immunity in the mouse. Br. J. Nutr. 72, 899-909 (1994).

55. Arezes, J. et al. Hepcidin-induced hypoferremia Is a critical host defense mechanism against the siderophilic bacterium Vibrio vulnificans. Cell Host Microbe 17, 47-57 (2015).

56. Zeng, C. et al. Hepatic Hepcidin Protects against Polymicrobial Sepsis in Mice by Regulating Host Iron Status. Anesthesiology Online First, 10.1097/ALN.0000000000000466 (2014).

57. Sazawal, S. et al. Effects of routine prophylactic supplementation with iron and folic acid on admission to hospital and mortality in preschool children in a high malaria transmission setting: community-based, randomised, placebo-controlled trial. The Lancet 367, 133-143 (2006).

58. Mebrahtu, T. et al. Low-Dose Daily Iron Supplementation for 12 Months Does Not Increase the Prevalence of Malarial Infection or Density of Parasites in Young Zanzibari Children. J. Nutr. 134, 3037-3041 (2004).

59. Gwamaka, M. et al. Iron deficiency protects against aevere Plasmodium falciparum malaria and death in young children. Clin. Infect. Dis. 54, 1137-1144 (2012).

60. Koka, S. et al. Iron deficiency influences the course of malaria in Plasmodium berghei infected mice. Biochem. Biophys. Res. Commun. 357, 608-614 (2007).

61. Matsuzaki-Moriya, C. et al. A critical role for phagocytosis in resistance to malaria in iron-deficient mice. Eur. J. Immunol. 41, 1365-1375 (2011).

62. Casals-Pascual, C. et al. Hepcidin demonstrates a biphasic association with anemia in acute Plasmodium falciparum malaria. haematologica 97, 1695-1698 
63. de Mast, Q. et al. Assessment of Urinary Concentrations of Hepcidin Provides Novel Insight into Disturbances in Iron Homeostasis during Malarial Infection. J. Infect. Dis. 199, 253-262 (2009).

64. Portugal, S. et al. Host-mediated regulation of superinfection in malaria. Nat. Med. 17, 732-737 (2011).

65. Wang, H.-Z., He, Y.-X., Yang, C.-J., Zhou, W. \& Zou, C.-G. Hepcidin is regulated during blood-stage malaria and plays a protective role in malaria infection. $J$. Immunol. 187, 6410-6416 (2011).

66. Armitage, A. E., Pinches, R., Eddowes, L. A., Newbold, C. I. \& Drakesmith, H. Plasmodium falciparum infected erythrocytes induce hepcidin (HAMP) mRNA synthesis by peripheral blood mononuclear cells. Br. J. Haematol. 147, 769-771 (2009).

67. Huang, $\mathrm{H}$. et al. Interleukin-10 regulates hepcidin in Plasmodium falciparum malaria. PloS One 9, e88408 (2014).

68. Armitage, A. E. et al. Distinct patterns of hepcidin and iron regulation during HIV-1, HBV, and HCV infections. Proc. Natl. Acad. Sci. U. S. A. 111, 12187-12192 (2014).

69. Lambrecht, R. W. et al. Iron Levels in Hepatocytes and Portal Tract Cells Predict Progression and Outcomes of Patients With Advanced Chronic Hepatitis C. Gastroenterology 140, 1490-1500.e3 (2011).

70. Tung, B. Y. et al. Hepatitis C, iron status, and disease severity: Relationship with HFE mutations. Gastroenterology 124, 318-326 (2003). 
71. Fujita, N. et al. Hepcidin Expression in the Liver: Relatively Low Level in Patients with Chronic Hepatitis C. Mol. Med. 13, 97-104 (2007).

72. Aoki, C. A. et al. Liver hepcidin mRNA correlates with iron stores, but not inflammation, in patients with chronic hepatitis C. J. Clin. Gastroenterol. 39, 71-74 (2005).

73. Girelli, D. et al. Reduced serum hepcidin levels in patients with chronic hepatitis C. J. Hepatol. 51, 845-852 (2009).

74. Miura, K., Taura, K., Kodama, Y., Schnabl, B. \& Brenner, D. A. Hepatitis C virusinduced oxidative stress suppresses hepcidin expression through increased histone deacetylase activity. Hepatology 48, 1420-1429 (2008).

75. Nishina, S. et al. Hepatitis C virus-induced reactive oxygen species raise hepatic iron level in mice by reducing hepcidin transcription. Gastroenterology 134, 226238 (2008).

76. De Domenico, I. et al. Hepcidin mediates transcriptional changes that modulate acute cytokine-induced inflammatory responses in mice. J. Clin. Invest. 120, 23952405 (2010).

77. Domenico, I. D. et al. The Molecular Mechanism of Hepcidin-mediated Ferroportin Down-Regulation. Mol. Biol. Cell 18, 2569-2578 (2007).

78. Pagani, A. et al. Low hepcidin accounts for the proinflammatory status associated with iron deficiency. Blood 118, 736-746 (2011).

79. Riba, M. et al. A strong anti-inflammatory signature revealed by liver transcription profiling of Tmprss6-/- Mice. PloS One 8, e69694 (2013). 
80. Potrykus, J. et al. Fungal Iron Availability during Deep Seated Candidiasis Is Defined by a Complex Interplay Involving Systemic and Local Events. PLOS Pathog. 9, e1003676 (2013).

81. Broberg, C. A., Palacios, M. \& Miller, V. L. Klebsiella: a long way to go towards understanding this enigmatic jet-setter. F1000Prime Rep. 6, (2014).

82. Lawlor, M. S., Hsu, J., Rick, P. D. \& Miller, V. L. Identification of Klebsiella pneumoniae virulence determinants using an intranasal infection model. Mol. Microbiol. 58, 1054-1073 (2005).

83. Shon, A. S., Bajwa, R. P. S. \& Russo, T. A. Hypervirulent (hypermucoviscous) Klebsiella pneumoniae. Virulence 4, 107-118 (2013).

84. Struve, C., Bojer, M. \& Krogfelt, K. A. Characterization of Klebsiella pneumoniae Type 1 Fimbriae by Detection of Phase Variation during Colonization and Infection and Impact on Virulence. Infect. Immun. 76, 4055-4065 (2008).

85. Struve, C., Bojer, M. \& Krogfelt, K. A. Identification of a conserved chromosomal region encoding Klebsiella pneumoniae type 1 and type 3 fimbriae and assessment of the role of fimbriae in pathogenicity. Infect. Immun. 77, 5016-5024 (2009).

86. Moore, T. A. et al. Increased mortality and dysregulated cytokine production in tumor necrosis factor receptor 1-deficient mice following systemic Klebsiella pneumoniae infection. Infect. Immun. 71, 4891-4900 (2003).

87. Standiford, L. R. et al. TLR4-dependent GM-CSF protects against lung injury in Gram-negative bacterial pneumonia. Am. J. Physiol. - Lung Cell. Mol. Physiol. 302, L447-L454 (2012). 
88. Happel, K. I. et al. Cutting edge: roles of Toll-like receptor 4 and IL-23 in IL-17 expression in response to Klebsiella pneumoniae Infection. J. Immunol. 170, 4432$4436(2003)$.

89. Aujla, S. J. et al. IL-22 mediates mucosal host defense against Gram-negative bacterial pneumonia. Nat. Med. 14, 275-281 (2008).

90. Belaaouaj, A. et al. Mice lacking neutrophil elastase reveal impaired host defense against gram negative bacterial sepsis. Nat. Med. 4, 615-618 (1998).

91. Mehrad, B. et al. Transient Lung-Specific Expression of the Chemokine KC Improves Outcome in Invasive Aspergillosis. Am. J. Respir. Crit. Care Med. 166, 1263-1268 (2002).

92. Bettina, A. et al. M-CSF mediates host defense during bacterial pneumonia by promoting the survival of lung and liver mononuclear phagocytes. J. Immunol. 196, 5047-5055 (2016).

93. Chen, L., Zhang, Z., Barletta, K. E., Burdick, M. D. \& Mehrad, B. Heterogeneity of lung mononuclear phagocytes during pneumonia: contribution of chemokine receptors. Am. J. Physiol. Lung Cell. Mol. Physiol. 305, L702-711 (2013).

94. Podschun, R. \& Ullmann, U. Klebsiella spp. as Nosocomial Pathogens: Epidemiology, Taxonomy, Typing Methods, and Pathogenicity Factors. Clin. Microbiol. Rev. 11, 589-603 (1998).

95. Koczura, R. \& Kaznowski, A. Occurrence of the Yersinia high-pathogenicity island and iron uptake systems in clinical isolates of Klebsiella pneumoniae. Microb. Pathog. 35, 197-202 (2003). 
96. Nassif, X. \& Sansonetti, P. J. Correlation of the virulence of Klebsiella pneumoniae $\mathrm{K} 1$ and K2 with the presence of a plasmid encoding aerobactin. Infect. Immun. 54, 603-608 (1986).

97. Bachman, M. A. et al. Klebsiella pneumoniae yersiniabactin promotes respiratory tract infection through evasion of lipocalin 2. Infect. Immun. 79, 3309-3316 (2011).

98. Bachman, M. A., Lenio, S., Schmidt, L., Oyler, J. E. \& Weiser, J. N. Interaction of lipocalin 2, transferrin, and siderophores determines the replicative niche of Klebsiella pneumoniae during pneumonia. mBio 3, e00224-11 (2012).

99. Bachman, M. A., Miller, V. L. \& Weiser, J. N. Mucosal lipocalin 2 has proinflammatory and iron-sequestering effects in response to bacterial enterobactin. PLoS Pathog. 5, e1000622 (2009).

100. Chan, Y. R. et al. Lipocalin 2 Is required for pulmonary host defense against Klebsiella infection. J. Immunol. 182, 4947-4956 (2009).

101. Russo, T. A., Olson, R., MacDonald, U., Beanan, J. \& Davidson, B. A. Aerobactin, but Not Yersiniabactin, Salmochelin, or Enterobactin, Enables the Growth/Survival of Hypervirulent (Hypermucoviscous) Klebsiella pneumoniae Ex Vivo and In Vivo. Infect. Immun. 83, 3325-3333 (2015).

102. Russo, T. A. et al. Aerobactin Mediates Virulence and Accounts for Increased Siderophore Production under Iron-Limiting Conditions by Hypervirulent (Hypermucoviscous) Klebsiella pneumoniae. Infect. Immun. 82, 2356-2367 (2014).

103. Lawlor, M. S., O'Connor, C. \& Miller, V. L. Yersiniabactin is a virulence factor for Klebsiella pneumoniae during pulmonary infection. Infect. Immun. 75, 1463-1472 (2007). 
104. Brewer, R. J., Galland, R. B. \& Polk, H. C. Amelioration by muramyl dipeptide of the effect of induced hyperferremia upon Klebsiella infection in mice. Infect. Immun. 38, 175-178 (1982).

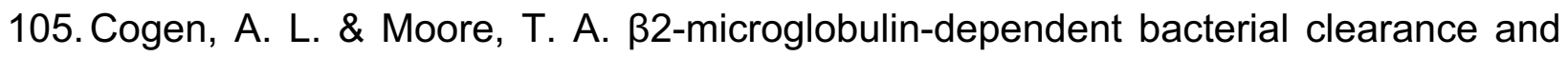
survival during murine Klebsiella pneumoniae bacteremia. Infect. Immun. 77, 360366 (2009).

106. Miles, A. A., Pillow, J. \& Khimji, P. L. The action of iron on local Klebsiella infection of the skin of the guinea-pig and its relation to the decisive period in primary infective lesions. Br. J. Exp. Pathol. 57, 217-242 (1976).

107. Denning, D. W. Invasive aspergillosis. Clin. Infect. Dis. 781-803 (1998).

108. Philippe, B. et al. Killing of Aspergillus fumigatus by alveolar macrophages is mediated by reactive oxidant intermediates. Infect. Immun. 71, 3034-3042 (2003).

109. Patterson, T. F. et al. Invasive aspergillosis disease spectrum, treatment practices, and outcomes. Medicine (Baltimore) 79, 250-260 (2000).

110. Herbrecht, R. et al. Voriconazole versus amphotericin B for primary therapy of invasive aspergillosis. N. Engl. J. Med. 347, 408-415 (2002).

111. Gersuk, G. M., Underhill, D. M., Zhu, L. \& Marr, K. A. Dectin-1 and TLRs permit macrophages to distinguish between different Aspergillus fumigatus cellular states. J. Immunol. 176, 3717-3724 (2006).

112. Mircescu, M. M., Lipuma, L., van Rooijen, N., Pamer, E. G. \& Hohl, T. M. Essential role for neutrophils but not alveolar macrophages at early time points following Aspergillus fumigatus infection. J. Infect. Dis. 200, 647-656 (2009). 
113. Park, S. J. \& Mehrad, B. Innate immunity to Aspergillus species. Clin. Microbiol. Rev. 22, 535-551 (2009).

114. Cenci, E. et al. Th1 and Th2 cytokines in mice with invasive aspergillosis. Infect. Immun. 65, 564-570 (1997).

115. Zelante, T. et al. Th17 cells in the setting of Aspergillus infection and pathology. Med. Mycol. 47, S162-S169 (2009).

116. Lass- Flörl, C., Roilides, E., Löffler, J., Wilflingseder, D. \& Romani, L. Minireview: host defence in invasive aspergillosis. Mycoses 56, 403-413 (2013).

117. Eisendle, M. et al. The intracellular siderophore ferricrocin Is involved in iron storage, oxidative-stress resistance, germination, and sexual development in Aspergillus nidulans. Eukaryot. Cell 5, 1596-1603 (2006).

118. Xiao, L. et al. Three-dimensional models of wild-type and mutated forms of cytochrome P450 14a-Sterol demethylases from Aspergillus fumigatus and Candida albicans provide insights into posaconazole binding. Antimicrob. Agents Chemother. 48, 568-574 (2004).

119. Shibuya, K. et al. Catalases of Aspergillus fumigatus and inflammation in Aspergillosis. Nippon Ishinkin Gakkai Zasshi 47, 249-255 (2006).

120. Schrettl, M. et al. Siderophore biosynthesis but not reductive iron assimilation Is essential for Aspergillus fumigatus virulence. J. Exp. Med. 200, 1213-1219 (2004).

121. Hissen, A. H. T. \& Moore, M. M. Site-specific rate constants for iron acquisition from transferrin by the Aspergillus fumigatus siderophores N', N", N"'triacetylfusarinine C and ferricrocin. JBIC J. Biol. Inorg. Chem. 10, 211-220 (2005). 
122. Eisendle, M., Oberegger, H., Zadra, I. \& Haas, H. The siderophore system is essential for viability of Aspergillus nidulans: functional analysis of two genes encoding I-ornithine N 5-monooxygenase $(\operatorname{sidA})$ and a non-ribosomal peptide synthetase (sidC). Mol. Microbiol. 49, 359-375 (2003).

123. Oberegger, H., Eisendle, M., Schrettl, M., Graessle, S. \& Haas, H. 4Phosphopantetheinyl transferase-encoding npgA is essential for siderophore biosynthesis in Aspergillus nidulans. Curr. Genet. 44, 211-215 (2003).

124. Schrettl, M. et al. Distinct roles for intra-and extracellular siderophores during Aspergillus fumigatus infection. PLoS Pathog. 3, e128 (2007).

125. Haas, H. et al. Characterization of the Aspergillus nidulans transporters for the siderophores enterobactin and triacetylfusarinine C. Biochem $J \mathbf{3 7 1}, 505-513$ (2003).

126. Kragl, C. et al. EstB-mediated hydrolysis of the siderophore triacetylfusarinine C optimizes iron uptake of Aspergillus fumigatus. Eukaryot. Cell 6, 1278-1285 (2007).

127. Wallner, A. et al. Ferricrocin, a siderophore involved in intra-and transcellular iron distribution in Aspergillus fumigatus. Appl. Environ. Microbiol. 75, 4194-4196 (2009).

128. Hortschansky, P. et al. Interaction of HapX with the CCAAT-binding complex-a novel mechanism of gene regulation by iron. EMBO J. 26, 3157-3168 (2007).

129. Schrettl, M. et al. HapX-mediated adaption to iron starvation is crucial for virulence of Aspergillus fumigatus. PLoS Pathog. 6, e1001124 (2010). 
130. Alexander, J., Limaye, A. P., Ko, C. W., Bronner, M. P. \& Kowdley, K. V. Association of hepatic iron overload with invasive fungal infection in liver transplant recipients. Liver Transpl. 12, 1799-1804 (2006).

131. Altes, A. et al. Frequent severe liver iron overload after stem cell transplantation and its possible association with invasive aspergillosis. Bone Marrow Transpl. 34, 505-509 (2004).

132. Kontoyiannis, D. P. et al. Increased bone marrow iron stores is an independent risk factor for invasive aspergillosis in patients with high-risk hematologic malignancies and recipients of allogeneic hematopoietic stem cell transplantation. Cancer 110, 1303-1306 (2007).

133. Zarember, K. A., Sugui, J. A., Chang, Y. C., Kwon-Chung, K. J. \& Gallin, J. I. Human polymorphonuclear leukocytes inhibit Aspergillus fumigatus conidial growth by lactoferrin-mediated iron depletion. J. Immunol. 178, 6367-6373 (2007).

134. Ellett, F., Jorgensen, J., Frydman, G. H., Jones, C. N. \& Irimia, D. Neutrophil interactions stimulate evasive hyphal branching by Aspergillus fumigatus. PLOS Pathog. 13, e1006154 (2017).

135. Ibrahim, A. S., Gebremariam, T., French, S. W., Edwards, J. E. \& Spellberg, B. The iron chelator deferasirox enhances liposomal amphotericin B efficacy in treating murine invasive pulmonary aspergillosis. J. Antimicrob. Chemother. 65, 289-292 (2010).

136. Conesa, A., van den Hondel, C. A. M. J. J. \& Punt, P. J. Studies on the production of fungal peroxidases in Aspergillus niger. Appl. Environ. Microbiol. 66, 3016-3023 (2000). 
137. Stewart, P., Whitwam, R. E., Kersten, P. J., Cullen, D. \& Tien, M. Efficient expression of a Phanerochaete chrysosporium manganese peroxidase gene in Aspergillus oryzae. Appl. Environ. Microbiol. 62, 860-864 (1996).

138. Ruiz-Dueñas, F. J., Martínez, M. J. \& Martínez, Á. T. Heterologous Expression of Pleurotus eryngii Peroxidase Confirms Its Ability To Oxidize Mn2+ and Different Aromatic Substrates. Appl. Environ. Microbiol. 65, 4705-4707 (1999).

139. Franken, A. C. W. et al. Analysis of the role of the Aspergillus niger aminolevulinic acid synthase (hemA) gene illustrates the difference between regulation of yeast and fungal haem- and sirohaem-dependent pathways. FEMS Microbiol. Lett. 335, 104-112 (2012).

140. Meatherall, B. L., Gregson, D., Ross, T., Pitout, J. D. D. \& Laupland, K. B. Incidence, risk factors, and outcomes of Klebsiella pneumoniae bacteremia. Am. J. Med. 122, 866-873 (2009).

141. Uslan, D. Z. et al. Age- and sex-associated trends in bloodstream infection: A population-based study in Olmsted county, Minnesota. Arch. Intern. Med. 167, 834-839 (2007).

142. Cassat, J. E. \& Skaar, E. P. Iron in Infection and Immunity. Cell Host Microbe 13, 509-519 (2013).

143. Besson-Fournier, C. et al. Induction of activin B by inflammatory stimuli upregulates expression of the iron-regulatory peptide hepcidin through Smad1/5/8 signaling. Blood 120, 431-439 (2012).

144. Michels, K., Nemeth, E., Ganz, T. \& Mehrad, B. Hepcidin and host defense against infectious diseases. PLOS Pathog 11, e1004998 (2015). 
145. Agbaht, K. et al. Bacteremia in patients with ventilator-associated pneumonia is associated with increased mortality: A study comparing bacteremic vs. nonbacteremic ventilator-associated pneumonia. Crit. Care Med. 35, 2064-2070 (2007).

146. Magret, M. et al. Bacteremia is an independent risk factor for mortality in nosocomial pneumonia: a prospective and observational multicenter study. Crit. Care 15, R62 (2011).

147. Barletta, K. E., Cagnina, R. E., Burdick, M. D., Linden, J. \& Mehrad, B. Adenosine A2B receptor deficiency promotes host defenses against Gram-negative bacterial pneumonia. Am. J. Respir. Crit. Care Med. 186, 1044-1050 (2012).

148. Sutherland, R. E., Olsen, J. S., McKinstry, A., Villalta, S. A. \& Wolters, P. J. Mast cell IL-6 improves survival from Klebsiella pneumonia and sepsis by enhancing neutrophil killing. J. Immunol. 181, 5598-5605 (2008).

149. Guida, C. et al. A novel inflammatory pathway mediating rapid hepcidinindependent hypoferremia. Blood 125, 2265-2275 (2015).

150. Langdon, J. M. et al. Hepcidin-dependent and hepcidin-independent regulation of erythropoiesis in a mouse model of anemia of chronic inflammation. Am. J. Hematol. 89, 470-479 (2014).

151. Armitage, A. E. et al. Induced disruption of the iron-regulatory hormone hepcidin inhibits acute inflammatory hypoferraemia. J. Innate Immun. 8, 517-528 (2016).

152. Lesbordes-Brion, J.-C. et al. Targeted disruption of the hepcidin 1 gene results in severe hemochromatosis. Blood 108, 1402-1405 (2006). 
153. Nagababu, E. et al. Iron-deficiency anaemia enhances red blood cell oxidative stress. Free Radic. Res. 42, 824-829 (2008).

154. Breuer, W. et al. The assessment of serum nontransferrin-bound iron in chelation therapy and iron supplementation. Blood 95, 2975-2982 (2000).

155. Wang, L. \& Cherayil, B. J. Ironing out the wrinkles in host defense: interactions between iron homeostasis and innate immunity. J. Innate Immun. 1, 455-464 (2009).

156. Georgopoulou, U., Dimitriadis, A., Foka, P., Karamichali, E. \& Mamalaki, A. Hepcidin and the iron enigma in HCV infection. Virulence 5, 465-476 (2014).

157. Nahon, P. et al. In alcoholic cirrhosis, low-serum hepcidin levels associate with poor long-term survival. Liver Int. 36, 185-188 (2016).

158. Harrison-Findik, D. D. et al. Iron-mediated regulation of liver hepcidin expression in rats and mice is abolished by alcohol. Hepatology 46, 1979-1985 (2007).

159. Kessler, S. M., Laggai, S., Kiemer, A. K., Barghash, A. \& Helms, V. Hepatic hepcidin expression is decreased in cirrhosis and HCC. J. Hepatol. 62, 977-979 (2015).

160. Ramos, E. et al. Minihepcidins prevent iron overload in a hepcidin-deficient mouse model of severe hemochromatosis. Blood 120, 3829-3836 (2012).

161. Preza, G. C. et al. Minihepcidins are rationally designed small peptides that mimic hepcidin activity in mice and may be useful for the treatment of iron overload. $J$. Clin. Invest. 121, 4880 (2011).

162. Krause, A. et al. LEAP-1, a novel highly disulfide-bonded human peptide, exhibits antimicrobial activity. FEBS Lett. 480, 147-150 (2000). 
163. Pigeon, C. et al. A new mouse liver-specific gene, encoding a protein homologous to human antimicrobial peptide hepcidin, is overexpressed during iron overload. $J$. Biol. Chem. 276, 7811-7819 (2001).

164. Nemeth, E. et al. IL-6 mediates hypoferremia of inflammation by inducing the synthesis of the iron regulatory hormone hepcidin. J. Clin. Invest. 113, 1271-1276 (2004).

165. Han, C. Y. et al. Hepcidin inhibits Smad3 phosphorylation in hepatic stellate cells by impeding ferroportin-mediated regulation of Akt. Nat. Commun. 7, (2016).

166. Pak, M., Lopez, M., Gabayan, V., Ganz, T. \& Rivera, S. Suppression of hepcidin during anemia requires erythropoietic activity. Blood 108, 3730-3735 (2006).

167. Duong-Nu, T.-M. et al. All three TonB systems are required for Vibrio vulnificus CMCP6 tissue invasiveness by controlling flagellum expression. Infect. Immun. 84, 254-265 (2016).

168. Elin, R. J. \& Wolff, S. M. The Role of Iron in Nonspecific Resistance to Infection Induced by Endotoxin. J. Immunol. 112, 737-745 (1974).

169. Mehrad, B. et al. The lupus-susceptibility locus, Sle3, mediates enhanced resistance to bacterial infections. J. Immunol. 176, 3233-3239 (2006).

170. Guo, Z. et al. Cutting edge: Membrane lymphotoxin regulates CD8+ T cellmediated intestinal allograft rejection. J. Immunol. 167, 4796-4800 (2001).

171. Grundya, M. A., Gorman, N., Sinclairb, P. R., Chorneyd, M. J. \& Gerharda, G. S. High throughput non-heme iron assay for animal tissues. J. Biochem. Biophys. Methods 59, 195-200 (2004). 
172. Rebouche, C., Wilcox, C. L. \& Widness, J. A. Microanalysis of non-heme iron in animal tissues. J. Biochem. Biophys. Methods 58, 239-251 (2004).

173. Oremland, M. et al. A computational model of invasive aspergillosis in the lung and the role of iron. BMC Syst. Biol. 10, 1 (2016).

174. Esposito, B. P. Labile plasma iron in iron overload: redox activity and susceptibility to chelation. Blood 102, 2670-2677 (2003).

175. Barletta, K. E. et al. Leukocyte compartments in the mouse lung: Distinguishing between marginated, interstitial, and alveolar cells in response to injury. $J$. Immunol. Methods 375, 100-110 (2012).

176. Misharin, A. V., Morales-Nebreda, L., Mutlu, G. M., Budinger, G. R. S. \& Perlman, H. Flow cytometric analysis of macrophages and dendritic cell subsets in the mouse lung. Am. J. Respir. Cell Mol. Biol. 49, 503-510 (2013).

177. Rotman, E. \& Seifert, H. S. The Genetics of Neisseria Species. Annu. Rev. Genet. 48, 405-431 (2014).

178. Lee, B. C. Isolation of an outer membrane hemin-binding protein of Haemophilus influenzae type b. Infect. Immun. 60, 810-816 (1992).

179. Morton, D. J. et al. Reduced severity of middle ear infection caused by nontypeable Haemophilus influenzae lacking the hemoglobin/hemoglobin-haptoglobin binding proteins (Hgp) in a chinchilla model of otitis media. Microb. Pathog. 36, 25-33 (2004).

180. Whitby, P. W., Seale, T. W., VanWagoner, T. M., Morton, D. J. \& Stull, T. L. The iron/heme regulated genes of Haemophilus influenzae: comparative transcriptional profiling as a tool to define the species core modulon. BMC Genomics 10, 6 (2009). 
181. Skaar, E. P., Humayun, M., Bae, T., DeBord, K. L. \& Schneewind, O. Iron-Source Preference of Staphylococcus aureus Infections. Science 305, 1626-1628 (2004).

182. Carrizo-Chávez, M. A., Cruz-Castañeda, A. \& de Jesús Olivares-Trejo, J. The frpB1 gene of Helicobacter pylori is regulated by iron and encodes a membrane protein capable of binding haem and haemoglobin. FEBS Lett. 586, 875-879 (2012).

183. Weissman, Z. \& Kornitzer, D. A family of Candida cell surface haem-binding proteins involved in haemin and haemoglobin-iron utilization. Mol. Microbiol. 53, 1209-1220 (2004).

184. Foster, L.-A. A. Utilization and cell-surface binding of hemin by Histoplasma capsulatum. Can. J. Microbiol. 48, 437-442 (2002).

185. Cadieux, B. et al. The Mannoprotein Cig1 Supports Iron Acquisition From Heme and Virulence in the Pathogenic Fungus Cryptococcus neoformans. J. Infect. Dis. 207, 1339-1347 (2013).

186. Huntoon, K. M. et al. The acute phase protein haptoglobin regulates host immunity. J. Leukoc. Biol. 84, 170-181 (2008).

187. Thomsen, J. H., Etzerodt, A., Svendsen, P. \& Moestrup, S. K. The HaptoglobinCD163-Heme Oxygenase-1 Pathway for Hemoglobin Scavenging. Oxid. Med. Cell. Longev. 2013, 1-11 (2013).

188. Delanghe, J. R. \& Langlois, M. R. Hemopexin: a review of biological aspects and the role in laboratory medicine. Clin. Chim. Acta 312, 13-23 (2001). 
189. Lin, T. et al. Synergistic Inflammation Is Induced by Blood Degradation Products with Microbial Toll-Like Receptor Agonists and Is Blocked by Hemopexin. J. Infect. Dis. 202, 624-632 (2010).

190. Yang, F. et al. Haptoglobin reduces lung injury associated with exposure to blood. Am. J. Physiol. - Lung Cell. Mol. Physiol. 284, L402-L409 (2003).

191. Vinchi, F. et al. Hemopexin therapy reverts heme-induced pro-inflammatory phenotypic switching of macrophages in a mouse model of sickle cell disease. Blood blood-2015-08-663245 (2015). doi:10.1182/blood-2015-08-663245

192. Sakamoto, K. et al. IL-22 Controls Iron-Dependent Nutritional Immunity Against Systemic Bacterial Infections. Sci. Immunol. 2, (2017).

193. RASMUS LARSEN, R. G. et al. A Central Role for Free Heme in the Pathogenesis of Severe Sepsis. Sci. Transl. Med. 2, (2010).

194. Ben Yaakov, D. et al. Identification and characterization of haemofungin, a novel antifungal compound that inhibits the final step of haem biosynthesis. J. Antimicrob. Chemother. 71, 946-952 (2016).

195. Stergiopoulou, T. et al. Host-Dependent Patterns of Tissue Injury in Invasive Pulmonary Aspergillosis. Am. J. Clin. Pathol. 127, 349-355 (2007).

196. Bettina, A. Host factors in defense against bacterial and fungal pneumonia. (University of Virginia, 2016).

197. Staples, C. A., Kang, E. Y., Wright, J. L., Phillips, P. \& Müller, N. L. Invasive pulmonary aspergillosis in AIDS: radiographic, CT, and pathologic findings. Radiology (1995). doi:10.1148/radiology.196.2.7617853 
198. Marr, K. A., Patterson, T. \& Denning, D. Aspergillosis. Pathogenesis, clinical manifestations, and therapy. Infect. Dis. Clin. North Am. 16, 875-894, vi (2002).

199. Schwienbacher, M. et al. Analysis of the major proteins secreted by the human opportunistic pathogen Aspergillus fumigatus under in vitro conditions. Med. Mycol. 43, 623-630 (2005).

200. Park, S. J. et al. Neutropenia enhances lung dendritic cell recruitment in response to Aspergillus via a cytokine-to-chemokine amplification loop. J. Immunol. 185, 6190-6197 (2010).

201. Riemer, J., Hoepken, H. H., Czerwinska, H., Robinson, S. R. \& Dringen, R. Colorimetric ferrozine-based assay for the quantification of iron in cultured cells. Anal. Biochem. 331, 370-375 (2004).

202. Lehmann, P. F. t \& White, L. O. Chitin assay used to demonstrate renal localization and cortisone-enhanced growth of Aspergillus fumigatus mycelium in mice. Infect. Immun. 12, 987-992 (1975).

203. Podschun, R. \& Ullmann, U. Klebsiella spp. as nosocomial pathogens: epidemiology, taxonomy, typing methods, and pathogenicity Factors. Clin. Microbiol. Rev. 11, 589-603 (1998).

204. Drancourt, M., Bollet, C., Carta, A. \& Rousselier, P. Phylogenetic analyses of Klebsiella species delineate Klebsiella and Raoultella gen. nov., with description of Raoultella ornithinolytica comb, nov., Raoultella terrigena comb, nov. and Raoultella planticola comb, nov. Int. J. Syst. Evol. Microbiol. 51, 925-932 (2001).

205. Gustot, T., Durand, F., Lebrec, D., Vincent, J.-L. \& Moreau, R. Severe sepsis in cirrhosis. Hepatology 50, 2022-2033 (2009). 
206. Jong, G.-M., Hsiue, T.-R., Chen, C.-R., Chang, H.-Y. \& Chen, C.-W. Rapidly fatal outcome of bacteremic Klebsiella pneumoniae pneumonia in alcoholics. Chest 107, 214-217 (1995).

207. Alcantara, R. B., Preheim, L. C. \& Gentry-Nielsen, M. J. Pneumolysin-Induced Complement Depletion during Experimental Pneumococcal Bacteremia. Infect. Immun. 69, 3569-3575 (2001).

208. Alcantara, R. B., Preheim, L. C. \& Gentry, M. J. Role of Pneumolysin's Complement-Activating Activity during Pneumococcal Bacteremia in Cirrhotic Rats. Infect. Immun. 67, 2862-2866 (1999).

209. Preheim, L. C., Snitily, M. U. \& Gentry, M. J. Effects of Granulocyte ColonyStimulating Factor in Cirrhotic Rats with Pneumococcal Pneumonia. J. Infect. Dis. $174,225-228(1996)$.

210. Preheim, L. C., Olsen, K. M., Yue, M., Snitily, M. U. \& Gentry-Nielsen, M. J. Effect of cirrhosis on antibiotic efficacy in a rat model of pneumococcal pneumonia. Diagn. Microbiol. Infect. Dis. 51, 103-111 (2005).

211. Carpenter, J. L. Klebsiella pulmonary infections: occurrence at one medical center and review. Rev. Infect. Dis. 12, 672-682 (1990).

212. Bridle, K. R. et al. Hepcidin Is Down- regulated in Alcoholic Liver Injury: Implications for the Pathogenesis of Alcoholic Liver Disease. Alcohol. Clin. Exp. Res. 30, 106-112 (2006).

213. Kell, D. B. \& Pretorius, E. Serum ferritin is an important inflammatory disease marker, as it is mainly a leakage product from damaged cells. Metallomics 6, 748773 (2014). 
214. Cohen, L. A. et al. Serum ferritin is derived primarily from macrophages through a nonclassical secretory pathway. Blood 116, 1574-1584 (2010).

215. Bugianesi, E. et al. Relative contribution of iron burden, HFE mutations, and insulin resistance to fibrosis in nonalcoholic fatty liver. Hepatology 39, 179-187 (2004).

216. Adamkiewicz, T. V. et al. Serum ferritin level changes in children with sickle cell disease on chronic blood transfusion are nonlinear and are associated with iron load and liver injury. Blood 114, 4632-4638 (2009).

217. Nielsen, P., Günther, U., Dürken, M., Fischer, R. \& Düllmann, J. Serum ferritin iron in iron overload and liver damage: Correlation to body iron stores and diagnostic relevance. J. Lab. Clin. Med. 135, 413-418 (2000).

218. Brodsky, I., Dennis, L. H., Kahn, S. B. \& Brady, L. W. Normal Mouse Erythropoiesis. Cancer Res. 26, 198-201 (1966).

219. Paris, S. et al. Catalases of Aspergillus fumigatus. Infect. Immun. 71, 3551-3562 (2003).

220. Kumar, A., Ahmed, R., Singh, P. K. \& Shukla, P. K. Identification of virulence factors and diagnostic markers using immunosecretome of Aspergillus fumigatus. J. Proteomics 74, 1104-1112 (2011).

221. Gardiner, D. M. \& Howlett, B. J. Bioinformatic and expression analysis of the putative gliotoxin biosynthetic gene cluster of Aspergillus fumigatus. FEMS Microbiol. Lett. 248, 241-248 (2005).

222. Scharf, D. H. et al. Biosynthesis and function of gliotoxin in Aspergillus fumigatus. Appl. Microbiol. Biotechnol. 93, 467-472 (2012). 
223. Tsitsigiannis, D. I. et al. Aspergillus Cyclooxygenase-Like Enzymes Are Associated with Prostaglandin Production and Virulence. Infect. Immun. 73, 4548-4559 (2005). 